\title{
Universiteit
}

Leiden

The Netherlands

\section{Delayed graft function in renal transplantation}

Boom, H.

\section{Citation}

Boom, H. (2005, January 19). Delayed graft function in renal transplantation. Retrieved from https://hdl.handle.net/1887/579

Version: $\quad$ Corrected Publisher's Version

License: $\quad$ Licence agreement concerning inclusion of doctoral thesis in the Institutional Repository of the University of Leiden

Downloaded from: $\quad$ https://hdl.handle.net/1887/579

Note: To cite this publication please use the final published version (if applicable). 


\section{DELAYED GRAFT FUNCTION IN RENAL TRANSPLANTATION}




\section{DELAYED GRAFT FUNCTION IN RENAL TRANSPLANTATION}

\section{Proefschrift}

ter verkrijging van

de graad van Doctor aan de Universiteit Leiden, op gezag van de Rector Magnificus Dr.D.D. Breimer,

hoogleraar in de faculteit der Wiskunde en Natuurwetenschappen en die der Geneeskunde, volgens besluit van het College voor Promoties te verdedigen op woensdag 19 januari 2005 klokke 16.15 uur

door

\section{Hendrik Boom}

geboren te Koudekerk aan den Rijn in 1963 


\title{
PROMOTIE COMMISSIE
}

\author{
Promotores: $\quad$ Prof.Dr.M.R.Daha \\ Prof.Dr.L.C. Paul $\dagger$ \\ Co-promotor: Dr.J.W. de Fijter \\ Referent: $\quad$ Prof. Dr. U. Frei \\ Charité-Universitätsmedizin Berlin, Deutschland \\ Overige leden: Prof.Dr.L.A.van Es \\ Prof.Dr. J.A. Bruijn \\ Prof. Dr. F.H.Claas \\ Prof.Dr. H.J.v. Bockel \\ Prof.Dr. R.J.Ploeg \\ Universiteit Groningen \\ Prof.Dr. E.K.J. Pauwels
}

ISBN 90-8559-020-5

Printed by Optima Grafische Communicatie, Rotterdam

Additional financial support by Astra Zeneca, Bard Benelux, Baxter, Boehringer Ingelheim, Bristol Myers Squibb , The Dutch Kidney foundation, Fresenius Kabi, Fresenius Medical Care, Genzyme Europe, Leo Pharma, Merck Sharp \& Dohme, Nycomed, Novartis, Novo Nordisk, Pfizer, Roche, Sanofi-Synthelabo, Servier is gratefully acknowledged. 
Ter nagedachtenis aan mijn vader Voor Janet, Oscar en Yizenne 


\section{CONTENTS}

Chapter 1 Introduction and outline of the thesis 9

Chapter 2 Delayed graft function influences renal function, but not survival 29

Kidney International, 2000; 58:859 - 866

Chapter 3 Delayed graft function is characterized by reduced functional mass measured by ${ }^{99 m}$ technetium mercaptoacetyltriglycine renography

Transplantation 2002; 74:203-208

Chapter 4 Calcium levels as a risk factor for delayed graft function

Transplantation 2004; 77:868-873

Chapter 5 The expression of caspase-3 and manganese SOD in distal tubules predicts post-transplant acute tubular necrosis or DGF Submitted

Chapter 6 Delayed graft function in renal transplantation 
INTRODUCTION AND OUTLINE OF THE THESIS 


\section{INTRODUCTION}

Acute renal failure (ARF) on the basis of acute tubular necrosis (ATN) was defined in the early days of the 20 th century by the German pathologist Hackstadt. His observations were based on the clinical evaluation of soldiers who sustained severe traumatic injury. However its significance was more or less neglected until the second world war, when Bywaters described the crush injury syndrome in victims of the London Blitz (1). Initially the underlying morphological changes in this kind of renal failure was thought to be related to distal tubular injury due to pigment toxicity and the term lower nephron nephrosis was introduced for this clinical condition (2).Later classic micro dissection studies in kidneys of rats showed that the dominant side of injury in ATN was the straight segment of the proximal tubules (S3 segment, pars recta) (3).

\section{Diversities of acute renal failure}

The diversity of definitions has hampered the analysis with regard to the incidence of ARF, as it may present with or without oliguria. Some studies defined ARF on the basis of elevated serum creatinine concentration; others referred to the increments above baseline serum creatinine levels or included only patients with ARF who required dialysis. ARF is caused by a variety of different etiologies and occurs in a variety of clinical settings. In daily clinical practice, ARF due to hypo-perfusion is one of the most common forms of ARF, it may account for 40 to 80 per cent of all cases of ARF, and if prolonged, pre-renal ARF may develop into ATN. Postrenal causes of ARF by ureter-, bladder neck or urethral obstruction, are less common causes of ARF and are encountered in only 2 to 10 per cent of all cases. Renal causes of ARF are diseases like acute and crescentic glomerulonephritis, hemolytic uremic syndrome, interstitial nephritis and ATN.

\section{Acute tubular necrosis}

Studies on patients with ARF due to ATN illustrate that different insults to renal function can be present simultaneously. The most common predisposing factor in the development of ATN appears to be renal ischemia resulting from prolonged oxygen deprevation resulting clinically in pre renal azotaemia. Sepsis is a major predisposing factor and nephrotoxins account for about 25 per cent of all cases of ATN (amino-glycosides, radiocontrast media, organic solvents, NSAID's and heavy metals). ATN can also be the result of heavy loads of haem pigment to be excreted by the kidney, such as in rhabdomyolysis or haemolysis (4). It has been estimated that some 40 to 60 per cent of cases of ATN occur in a postoperative or trauma related setting, while the remainders emerge in a medical setting. The causes of the renal failure in these circumstances are often multifactorial in origin. High-risk settings are abdominal aortic aneurysm surgery, severe burns, pancreatitis, and advanced chronic liver disease. Mortality associated with ATN was reported to be between $37 \%$ and $79 \%$ (5). 


\section{Pathophysiology of renal failure in ATN}

One of the possible mechanisms of the decreased GFR in ARF due to ATN is related to tubular damage resulting from ischemia/reperfusion injury. Degeneration, necrosis and exfoliation of tubular epithelium, interstitial edema and interstitial cellular infiltration are usually observed in biopsies in ARF (6). There are two possible explanations for the impaired glomerular filtration rate.

One of the possible mechanisms of decreased GFR is tubular obstruction and tubular backleakage or there is a maladaptive response of the tubuloglomerular feedback loop.

Tubular obstruction and backleakage: In the early phase of ATN, tubular obstruction by exfoliated tubular cells and protein precipitates results in tubular obstruction and elevated proximal and distal intratubular pressures and a low net filtration pressure (7). Although obstruction is morphologically hard to demonstrate, since it can be present on many levels of the nephron, high intratubular pressures can be demonstrated in animal models $(8,9)$. Increased intratubular pressures are always present in cases of ischemic ATN but they do not sustain, as they decline in a period of days to normal or even low levels. Lowering of intratubular pressure is not uniformly associated with recovery of renal function. Another possibility, associated with the obstruction theory, is the existence of backleakage. Backleakage of glomerular filtrate was demonstrated and associated with severe tubular dysfunction in animal models $(10,11)$. Myers et al demonstrated the existence of backleakage in human ATN (12). The underlying pathophysiological mechanism is the disruption of the intercellular tight junctions in damaged tubular cells. Although it explains the impaired glomerular filtration rate in more severe ATN cases (12) it does not explain the decline of GFR in milder cases of ATN.

Decreased tubuloglomerular feedback: Another explanation for the relation between decline in glomerular filtration and tubular damage, is the reduction in renal bloodflow under the influence of decreased sodium and chloride reabsorption. The supply of chloride and sodium to the juxtaglomerular apparatus leads to an afferent and efferent vasoconstriction and diminished renal blood flow and glomerular filtration pressures $(13,14)$. This can be seen as a maladaptive mechanism of a teleologically appropriate mechanism of the kidney to prevent excessive loss of fluid and electrolytes (15).

\section{ARF in renal transplantation}

In renal transplantation a delayed start of graft function (DGF) is found in 10-50\% of cases (16-21). Literature on the pathophysiology and morphology of ARF in transplanted kidneys is scarce. Although risk factors for DGF and ARF in native kidneys are different in transplanted kidneys than in native kidneys, the underlying morphological and pathophysiological characteristics are often considered to be similar. The prevailing views are mainly based on studies of acute tubular renal failure in experimental animals (22).

In renal transplantation risk factors for delayed renal function immediately after transplantation are well known. They can be divided into donor linked factors, factors related to the transplantation procedure and factors related to the post-transplant period. These clinical conditions are unique for the transplantation setting and therefore differ substantially from the conditions in which ARF occurs in native kidneys. This makes the comparison with ARF 
in native kidneys hazardous. Experimental data on ischemia and reperfusion injury are also difficult to interpret since in these conditions the kidney usually is totally deprived from perfusion by clamping the renal artery. Despite these limitations, the literature on ischemic ARF in native kidneys and on experimental ATN could contribute to our understanding of the pathophysiology of DGF and its meaning for graft outcome and for the development of specific therapeutic interventions.

\section{Definition of DGF}

In most studies DGF is defined as the need of dialysis treatment in the first week. This is a criterion that is easy to register objectively and to obtain from large databases. However, dialysis during the first week after transplantation is also performed for other reasons than DGF, like hyperkaliemia and fluid overload. Others define for that reason DGF as a functional parameter distinct from the need of dialysis and use the time needed to achieve an arbitrarily defined creatinine clearance as a marker for DGF. Since, the pathogenesis of DGF is supposed to be of ischemic origin it is relevant to define DGF as a functional abnormality distinct from the need of dialysis treatment. In addition acute rejection should be excluded as a cause of DGF. We propose to use a functional definition using the decrease of serum creatinine of more than $10 \%$ per day for at least 3 consecutive days for more than 1 week after transplantation and excluding acute rejection and calcineurin inhibitor toxicity as a possible cause of this DGF. Using this definition, it is possible to analyze the risk factors and consequences of ischemic damage and associated reperfusion injury that is supposed to be the underlying cause of DGF and excluding changes of serum creatinine concentration by other causes like dilution, surgical complications, cyclosporin toxicity and acute rejection.

\section{DGF and long-term graft survival}

The effect of DGF on short term and long term patient and graft survival is still controversial. Some authors found a deleterious effect effect of DGF on graft survival $(20,23)$ and others did not or only found this effect when it coincided with acute rejection episodes (24). These discrepancies may be related to the criteria used to define DGF or to differences in data analysis. Most authors used the need for dialysis within the first week as the diagnostic inclusion criterion but this does reflect the various causes of DGF such as ischemia- reperfusion injury, early acute rejection episodes or calcineurin inhibitor toxicity. In the UNOS registry, DGF was defined as the need for dialysis in the first week after transplantation. It had a significant and independent impact on graft half-life. This effect was independent from cold ischemia time, occurrence of acute rejection episodes, donor age and serum creatinine levels (25). Others found a detrimental effect of DGF, also defined as the need for dialysis in the first week, on graft survival only when it was complicated by one or more acute rejection episodes $(24,26,27)$. When the time required to reach a Cockroft renal clearance of more than $10 \mathrm{ml} / \mathrm{min}$ was used, DGF lasting for more than 6 days had a deleterious effect on graft survival whereas DGF of shorter duration did not influence graft survival (28).

Looking at the different survival curves it is striking that one year after transplantation the survival curves essentially run parallel. This suggests an effect of DGF on graft survival wit- 
hin the first year but no effect beyond. This is supported by studies that analyzed the risk factors on graft survival at different time intervals after transplantation and found that progression of chronic graft failure is mainly associated with donor age, creatinine clearance at 1 year after transplantation, proteinuria and the presence of hypertension in the recipient and not with the occurrence of DGF.

\section{Risk factors of DGF}

Risk factors for DGF can be divided in donor-related factors, transplantation-related factors and recipient related risk factors. The cadaveric kidney is subject to damage at every step along the way from procurement to reperfusion whereas kidneys from living donors rarely develop DGF.

\section{Donor related risk factors}

Well-known donor related risk factors for DGF are donor age over 50 years and an elevated serum creatinine or decreased renal function of the donor. In human adults total metabolism and renal function in terms of glomerular filtration rate and renal blood flow and muscle mass decrease with age. This implies that for the same serum creatinine concentration GFR in the elderly can be severely impaired in comparison to younger adults. Kidneys from older individuals may have several structural and functional changes compared with kidneys from younger donors. Longitudinal studies of elderly individuals have shown a diminution in renal reserve, along with functional constraints on the kidney's ability to respond appropriately to challenges of either excesses or deficits (29). Studies of kidneys obtained at autopsies demonstrated a progressive decrease in the number and size of glomeruli with age, resulting in a progressive decrease of the glomerular filtration volume $(30,31)$. In addition to the loss of glomeruli, there is an age-dependent increase in the cortical interstitial volume as a result of progressive interstitial fibrosis $(31,32)$. Most renal biopsies from kidney donors who are older than $40 \mathrm{yr}$ show intimal fibrosis in the smaller arteries, arteriolar hyalinosis, and interstitial fibrosis (33).

Not only factors intrinsically related to the donor, but also the events prior to brain death and harvest of the kidney contribute to the occurrence of DGF. Before the establishment of brain death of the potential donor, the kidney may be damaged by the underlying disease process, or by the therapeutic maneuvers instituted in an attempt to revive the patient or to maintain circulation after brain death, like the use of dopaminergic medication and resuscitation procedures. Decreasing platelet count and disseminated intravascular coagulation are frequent and suggest that endothelial injury or dysfunction may already be present in the postmortal donor. During episodes of cardiac arrest or prolonged hypotension, the kidney will suffer from warm ischemia and reperfusion injury. Catecholamine release and pharmacological inotropes may contribute to intrarenal vasospasms leading to relative hypoperfusion. The donor will be in a catabolic state, making recovery from ischemic damage more difficult. Finally many donors have evidence of intravascular coagulation, which may be either a cause or a consequence of endothelial injury in the microcirculation. In addition after brain death but before explantation, the patient may not be considered a high priority for surgery at a busy intensive care unit and resuscitation may be delegated to those with limited experience in appropriate care (34). 


\section{Risk factors related to the transplantation procedure}

Organ procurement also contributes to the development of DGF. This starts with hypoperfusion after circulation of the donor has stopped (warm ischemia time (WIT)). With the multi organ procurement procedures this WIT is limited to several minutes. However, with the raising interest of non-heart beating procedures, WIT becomes a serious contributor to DGF $(35,36)$. During surgery errors in line placement can result in inadequate flushing of blood and cooling, and undue manipulation of renal arteries can induce vascular spasm. The most important independent and robust risk factor is cold ischemia time (CIT) (18,3742). During preservation the kidney is exposed to ATP depletion probably enhanced by reperfusion induced vasoconstriction, resulting in apoptosis and necrosis of individual cells and leading to severe functional damage (43). The type of preservation fluid also has been recognized as a risk factor for DGF in a study in the Eurotransplant area, in which the preservation fluid developed by the University of Wisconsin (UW) appeared to be superior to Euro Collins (EC) (44). Cold pulsatile perfusion in which organs are perfused with a pulsatile preservation machine are described to have a lower incidence of DGF than organs that are preserved with a cold flush (40).

After perfusion is re-established several mechanisms exist that can damage the renal allograft including the generation of free radicals, mechanical injury to blood vessels from sudden high blood flow, vasomotor derangement from prostaglandins and other regulatory peptide imbalances and cytokine release from inflammatory infiltrates (45)

\section{Recipient related risk factors}

Recipient age is a risk factor for DGF especially when kidneys from pediatric donors to adult recipients are involved (20). The relation between the occurrence of DGF and the discrepancy between donor and recipient Body Mass Indexes (BMI) supports this hypothesis (46). Secondary hyperparathyroidism is also associated with a higher incidence of DGF $(47,48)$. The lower occurrence rate of DGF with zero HLA mismatch and low levels of panel reactive antibodies (PRA) (49), suggests that immunology related factors are involved in the development of DGF. Since the studies that describe this effect, used dialysis treatment in the first week as their definition of DGF, it is very likely that early acute rejection activity is the missing link $(20,40,50)$. In addition, the use of calcineurin inhibitors is a riskfactor because their vasoconstrictive properties influence renal perfusion and enhance ischemic damage (51).

\section{Morphological characteristics of ATN in native kidneys and grafts}

Changes in renal morphology that accompany ARF in native kidneys are subtle $(52,53)$. In renal biopsies from patients suffering ARF, regeneration and necrosis coexist, suggesting that the injury and repair process coincide. Solez studied 57 biopsies from patients with ARF and defined twelve characteristic morphological items for ATN $(52,53)$. These items represent the morphological consequences of necrosis and regeneration respectively. Markers for necrosis were: the presence of individual necrosis, loss of brush border, tubular dilatation and the presence of tubular casts, signs of interstitial inflammation and edema, hyperplasia of the juxta glomerular apparatus, dilatation of Bowman's space, tubularization of the parietal epithelial cells of Bowman's capsule. As signs of recovery the presence of mitotic figures and signs of tubular regeneration were studied. Also the accumulation of 
leukocytes in the vasa recta were scored as signs of ATN. All items were scored on a 4 point scale from score 0 representing the absence of the item to score $3+$, representing abundant presence of the item. Only the presence of necrosis of individual tubular cells and the loss of the brush border were specific for oliguric ARF compared to biopsies from patients that had recovered recently from ARF (52). ARF after renal transplantation has a distinctly different etiology than ARF in native kidneys. Therefore the same group of investigators compared the above mentioned 57 biopsies with 13 allograft biopsies from patients with DGF and 5 biopsies from grafts with a stable function. The most striking difference between the graft biopsies with DGF and the biopsies from native kidneys with ARF due to ATN was that the non-replacement phenomenon was seen more often in the grafts. They also had larger interstitial infiltrates than the native kidneys with ATN. Furthermore grafts with DGF showed slightly less frequent disappearance of the tubular brush border, fewer tubular casts, less dilation of the Bowman's space but a greater number polarizable oxalate crystals $(6,54)$. Another striking feature in biopsies of ATN is the presence of an interstitial infiltrate. It is supposed to be related of the process of brain death (55) and ischemia/reperfusion injury leading to a local inflammatory reaction which is possibly associated with the production of free oxygen radicals $(56,57)$. Ischemia and reperfusion injury is associated with an upregulation of pro-inflammatory cytokines like interleukines (IL-1, IL-6, IL-8 and IL10) and monocyte chemoattractant protein 1 (MCP-1) (58-60). As a consequence adhesion molecules that are important in the migration of leucocytes, like intercellular adhesion molecule-1 (ICAM-1), vascular adhesion molecule (VCAM) and endothelial leukocyte adhesion molecule-1 (ELAM-1) $(61,62)$ also are upregulated.

Marcussen concluded there were no differences between biopsies with ATN of native and transplanted kidneys, as far as the composition of the interstitial infiltrates is concerned (6) but that in contrast to acute rejection episodes the infiltrate in ATN consisted mainly of granulocytes. Haug and Dragun showed in animal models that the detrimental effects of ischemia and reperfusion could be minimized with neutropenia and the use of blocking anti-bodies against ICAM-1, LFA-1 and P-selectin (63) or ICAM-1 antisense oligodesoxynucleotides (64).

\section{Pathogenesis of acute ischemic renal injury and subsequent recovery}

In the pathogenesis of acute ischemic failure 3 stages can be recognized. The first stage is the ischemic phase in which ischemic and reperfusion injury takes place and in which renal epithelial and endothelial cells are subjected to lethal insults leading to apoptosis and /or necrosis (65). The maintenance phase represents a phase of stabilization of injury by intrinsic or upregulated defense mechanisms. During this phase events leading to cellular repair, proliferation and redifferentiation. lead to the recovery phase in which epithelial en endothelial function improve, leading to the recovery of renal function. 


\section{Ischemia and reperfusion in ATN}

\section{a. Molecular biological characteristics}

Ischemic phase

During the ischemic phase, renal tissue suffers from the lack of oxygen and nutritional substrates whereas cell metabolism continues, resulting in decreased adenosine triphosphate (ATP) levels and a decreased supply of adenosine diphosphate (ADP) to the mitochondria (66). The shift to anaerobic metabolism results in the accumulation of lactic acid and a decrease in $\mathrm{pH}$. One of the most energy intensive cell functions is sodium and water homeostasis via the sodium-potassium pumps (67). When these pumps fail cellular, mitochondrial and nuclear swelling occurs. Increased extracellular potassium stimulates calcium ion channels, increasing cytosolic and mitochondrial calcium concentrations (68). Cytosolic calcium levels activate calcium dependent enzymes like cystein proteases, the phospholipases and endothelial nitric oxide synthetase (e-NOS) (69). Calpain is an example of a calcium dependent cystein protease capable of disrupting the cellular polarity by breaking down the integrity of the organizational proteins spectrin and ankyrin whereas caspase- 3 is a calcium dependent cystein protease playing a major role in programmed cell death. Phospholipase- 2 is able to change tubular cell polarity by breaking down cytoskeleton matrix and it plays a role in the synthesis of reactive oxygen species (ROS). The role of e-NOS is dual since it has been proven to be tubulotoxic (70), but also causes vascular dilation (71).

In this ischemic condition, hypoxanthine is the most damaging degradation product. It accumulates in the cell as a byproduct of the conversion of ATP to inosine. Under aerobic conditions hypoxanthine is metabolized via the production of xanthine to uric acid but in anaerobic conditions hypoxanthine accumulates in the endothelial and tubular cells. Xanthine oxidase is produced by proteolysis of the xanthine dehydrogenase under the influence of calcium dependent proteases (72).

\section{Reperfusion phase}

After the reinstitution of perfusion several factors contribute to the damage. The sudden increase of perfusion pressure causes endothelial damage and inflammation. Because of unequal distribution of perfusion, ischemia may persist in underperfused areas. Two types of molecules have been widely studied and have been implicated in ischemia and reperfusion injury; i.e. endothelin and Nitric Oxide (NO), which both modulate vascular tone. During ischemia systemic endothelin levels are elevated whereas anti-endothelin antibodies or endothelin receptor antagonists protect against ischemia and reperfusion injury $(73,74)$. Many of the actions of endothelin are counteracted by constitutively expressed and endothelin induced Nitric Oxide (NO). NO causes vasodilatation which protects against ischemic renal injury (75), but on the other hand it also is toxic for tubular cells $(70,71)$. This toxicity is probably caused by one of its metabolites, peroxynitrite, , which is a highly reactive oxidant resulting from the interaction between $\mathrm{NO}$ and the superoxide anion. During reperfusion molecular oxygen $\left(\mathrm{O}_{2}\right)$ is reintroduced (fig. 1 ). $\mathrm{O}_{2}$ reacts with the hypoxanthine that has accumulated in the cells during the ischemia phase. This reaction is mediated by xanthine oxidase and produces xanthine and reactive oxygen species (ROS). ROS refers to any compound derived from molecular oxygen that has acquired less than 4 electrons. The free radical members of ROS are Superoxide $\left({ }^{\circ} \mathrm{O}_{2}\right)$ and the hydroxyl radical $(\cdot \mathrm{OH})$. They are 
Figure 1.Schematic representation of the ischemia and reperfusion injury cascade Ischemia Reperfusion

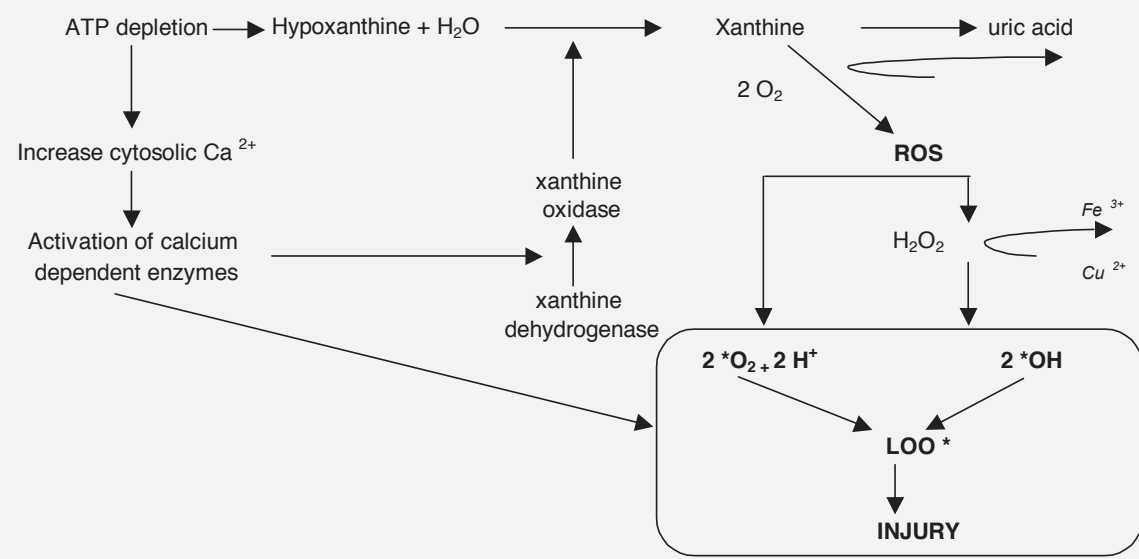

A period of oxygen deprivation results in a deprivation of cellular ATP. Hypoxanthine accumulates in the cell as a byproduct of the anaerobic conversion of ATP to inosine. Xanthine oxidase catalyses the reaction of hypoxanthine and oxygen to form reactive oxygen species (ROS). The free radical members of $\mathrm{ROS}, \mathrm{O}_{2}$ and ${ }^{\circ} \mathrm{OH}$ are the most harmful ones. They lead to lipid peroxidation in the cellular membrane, eventually leading to cell death by destruction of the cellular walls.

probably the most important biologically active free radicals: $\mathrm{O}_{2}$ is formed by transfer of a single electron to $\mathrm{O}_{2}$ and $\cdot \mathrm{OH}$ is formed from hydrogen peroxide $\left(\mathrm{H}_{2} \mathrm{O}_{2}\right)$. The latter reaction is spontaneous but is accelerated in the presence of a catalyzing transitional metal ion, for example Iron ( $\mathrm{Fe}^{3+}$ ) or Copper $\left(\mathrm{Cu}^{2+}\right)$.

The free radicals lead to an oxidative reduction of the poly unsaturated fatty acids (PUFA's) in the plasma membranes, known as lipid peroxidation. This lipid peroxidation leads to the production of fatty acid radicals and by reacting with oxygen to fatty acid peroxyl radicals (LOO*) These LOO's are the central players in a chain reaction that leads to further lipid peroxidation and the formation of reactive aldehydes. The harmful effect of the production of free radicals lies in the extensive damage to the cell membrane, leading to decreased function and/or cellular apoptosis or cell death (76-79)

\section{b. Cell biological characteristics}

Ischemia and reperfusion eventually lead to tubular cell death. Two types of cellular death can be distinguished: cells may die either by necrosis or by apoptosis. The morphological characteristics of apoptosis and necrosis are both quite distinct and remarkably constant among all kind of different cell types. 


\section{Apoptosis}

In contrast to necrosis, apoptosis is an active, energy dependent process with morphological characteristics that differ markedly from necrosis. Epithelial cells dying by apoptosis detach early from the underlying matrix and from each other. They become progressively smaller in size and their nuclear chromatin becomes condensed and fragmented. The plasma membrane remains intact and undergoes a process called blebbing. Ultimately, the apoptotic cell disintegrates into many membrane-bound vesicles, some of which contain fragments of condensed chromatin, the so-called apoptotic bodies. Apoptosis is a form of programmed cell death which is used physiologically to remove unwanted cells. The essence of apoptosis is a process of cellular auto-digestion.

Three stages can be recognized in the process of apoptosis (fig.2).

The first stage is the regulator stage. Regulator molecules control adaptor proteins by directly interacting with them. For example FADD (Fas associated death domain) activation can be inhibited by cellular FLIP (FLICE (Fas associated death domain like IL-1 beta converting enzyme) inhibitory protein). Apaf-1 (apoptotic protease activating factor 1 ) activation can be prevented by binding to the anti-apoptotic members of the $\mathrm{Bcl}-2$ families. Furthermore inhibitors of apoptosis proteins (IAP) can directly prevent caspase activation.

The second stage in the apoptosis process is the adaptor stage. The adaptor molecules are able to activate the caspases by binding to specific sides and therefore leading to proteolysis of the pro-enzyme. Adaptor molecules are up-stream caspases, like caspase 8 and caspase 9, that activate caspase 3 as the final common caspase. Caspase- 8 becomes activated by binding to the FADD adaptor protein and caspase- 9 is activated by it's adaptor protein Apaf-1.

Figure 2. Schematic representation of the apoptotic cascade

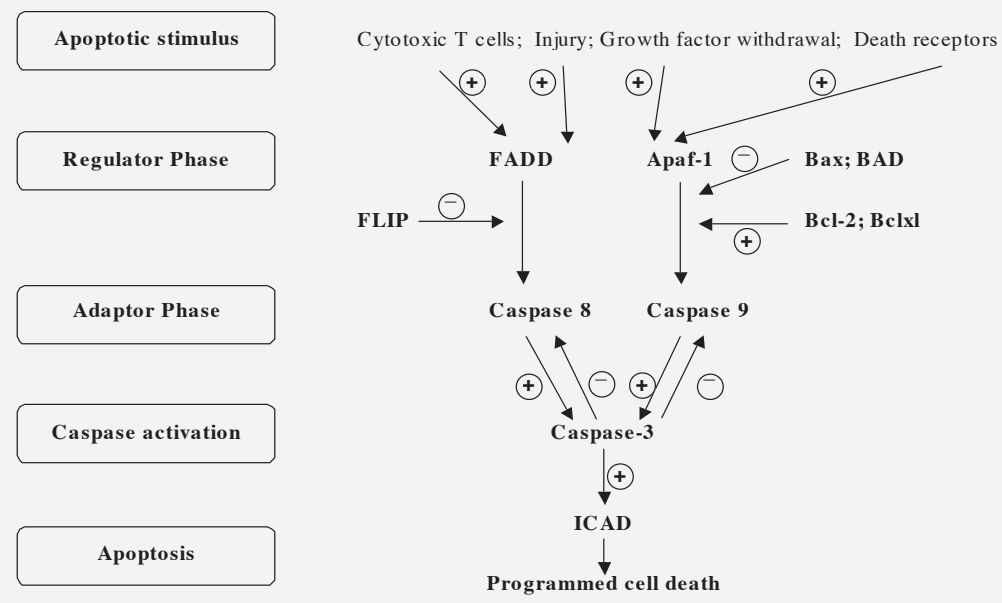

Apoptosis can be initiated by multiple signals. The strength of the apoptotic signal is evaluated by specific control proteins which can either inhibit or promote cell death. When the apoptotic signal is strong enough, caspase activation degrades cytoskeletal and nuclear proteins. This results in a cascade of intracellular degradation, eventually leading to the formation of apoptotic bodies and the engulfment of apoptotic material by phagocytic cells. 
The last stage is the effector stage. The key effector molecules are proteases named caspases of which the caspase-3 is the effector enzyme. Caspases are present in an inactivated form. Once they are activated the target of these activated caspases is ICAD (inhibitor of caspase activated D-nase (CAD). CAD is an endogenous endo-nuclease that fragments DNA of cells. Once activated CAD leads to fragmentation of the cellular DNA. Hence DNA fragmentation can be used as a marker of apoptosis.

\section{Necrosis}

Frank necrosis is seen in experimental animal models but only in a minority of the human cases. It is usually patchy involving individual cells or small clusters of cells sometimes resulting in small areas of denuded basement membrane (non-replacement phenomenon). Tubular cell necrosis is associated with a rapid metabolic collapse, cell swelling and early loss of plasma membrane integrity and the loss of polarity. Integrity of their tight junctions is disrupted, perhaps as a consequence of alterations in the cytoskeletal network $(67,80)$. Because of the redistribution of the Na/K-ATPases, tubular function is disturbed and cells die. This in turn leads to the release of proteolytic enzymes and other injurious cytosolic components into the extracellular space that not only directly damage surrounding cells but also incites an inflammatory response. More subtle changes include loss of brush border, flattening of the epithelium, detachment of cells, intra-tubular cast formation and dilation of the tubules (fig.3).

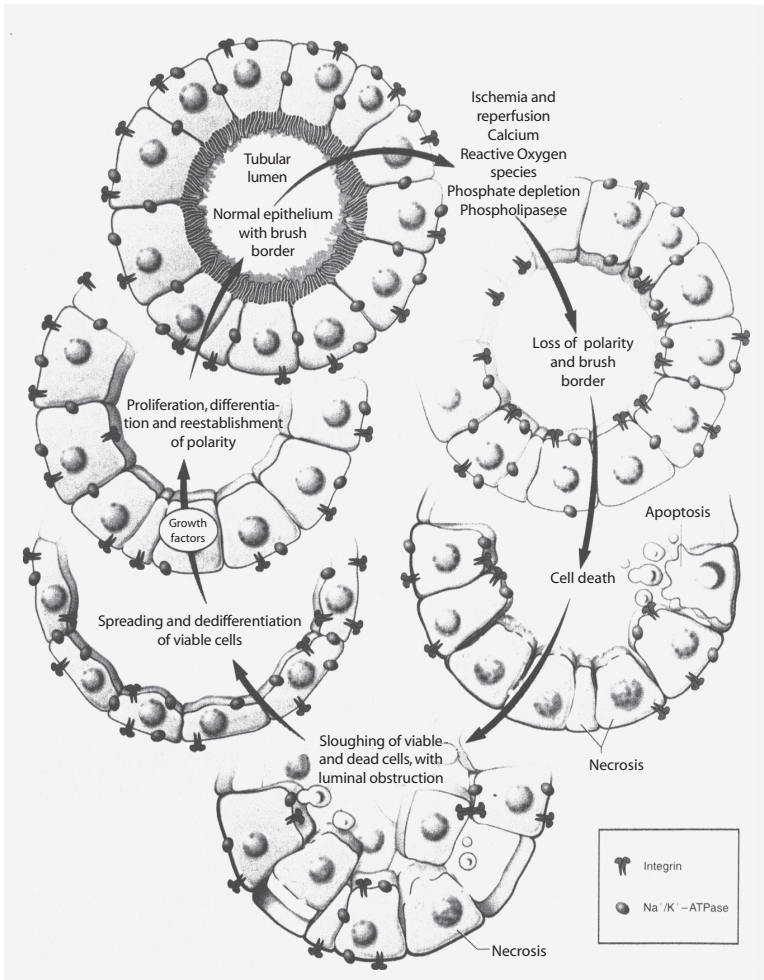

Figure 3.

Cell biological characteristics of acute tubular necrosis

Cells undergoing acute tubular necrosis are morphologically characterized by loss of brush border, flattening of the epithelium (distalisation of proximal tubular cells) and detachment of necrotic cells leading to intratubular cast formation and dilation of the tubules. This makes distal tubules hard to be discriminated from the proximal ones on morphological grounds. [Reprinted with permission (96)] 


\section{Maintenance phase of ATN}

The maintenance phase represents a phase of stabilization of injury in which lethal factors, characteristic for the ischemic stage, on the one hand and repair and survival factors, characteristic for the regeneration phase, on the other hand, are interacting and subsequent leading to cellular repair, division and redifferentiation.

\section{a. Molecular biological characteristics}

The kidney has naturally occurring anti-oxidant enzymes to counteract the effects of the free radicals. The catalases and gluthathion peroxidase act by safely decomposing the peroxides. Catalase is located mainly in the peroxisomes of the cells and therefore acts mainly upon hydrogen peroxidase whereas gluthation peroxidase is located in the cellular cytosol and therefore acts mainly on the hydroperoxides, that are derived from membrane's fatty acids. The superoxide dismutases act by scavenging the free radicals especially the ${ }^{\circ} \mathrm{O}_{2}$. In humans it is present in at least two forms, the cytoplasmatic copper/zinc (CuZn)-SOD and the mitochondrial manganese (Mn)-SOD. Superoxide dismutases are enzymes that catalyze the dismutation of $\cdot \mathrm{O}_{2}$ to hydrogen peroxide $\left(\mathrm{H}_{2} \mathrm{O}_{2}\right)$, which is decomposed, by other enzymes like catalase and gluthation peroxidase (76).The presence and the (down regulated) activity of these SOD's seem to be related to the amount of damage induced by ROS in rat kidneys $(81,82)$. However, the clinical use of human recombinant superoxide dismutase, did not protect against DGF in human kidneys $(83,84)$, although a decrease of acute rejection episodes (ARE) and chronic rejection was observed during follow up (85).

\section{b. Cell biological characteristics}

During the maintenance phase tubular cells share characteristics of the ischemic phase in which necrosis, apoptosis and the interstitial infiltrate are present and characteristics of the recovery and regeneration phase in which proliferation and redifferentiation are present.

\section{Recovery phase of ATN}

\section{a. Molecular biological characteristics}

This process is regulated by the expression of a number of transcription factors, structural proteins and growth factors and recapitulates many aspects of renal organo-genesis in respect to the high rate of DNA synthesis, like PCNA, the expression of apoptosis and the expression of genes that encode for processes during renal organogenesis like keratin and vimentin (86-88). The latter is a filament protein expressed in mesenchymal cells but not in mature nephrons. Whether these cells are derived by regression of surviving epithelial cells or by the activation of resident, but quiescent renal stem cells is not clear yet. The role of the induction of this gene expression after acute renal injury lies probably in their involvement in determining cell fate (survival versus apoptosis or necrosis) and the transcription of growth factors and their receptors that ultimately mediate tubular cell division and proliferation. Upon ischemia and reperfusion injury growth factors like the hepatic growth factor, insulin like growth factors and fibroblast growth factors are upregulated $(89,90)$. Others like epidermal growth factors are down regulated in injured proximal tubules (91). Treatment with these growth factors in 
animal models were promising $(89,92,93)$ but the use in humans is still limited $(94)$.

\section{b. Cell biological characteristics}

During recovery from ischemia and reperfusion injury, surviving tubular epithelial or renal stem cells, differentiate and proliferate, eventually replacing the irreversibly injured tubular epithelial cells and restoring tubular integrity (56). Morphologically this is characterized by the presence of mitotic figures and signs of cell proliferation. This process enables the replacement of the damaged epithelium and is maximal at 24 to 48 hours after ischemic injury in the rat (95). Initially proximal tubules reappear in a flattened fashion covering the tubular basement membrane, followed by the reappearance of the brush border. As the number of cells increase the tubular cells become more cubic in shape.

\section{Aim of the thesis}

The purpose of this thesis is to evaluate the mechanisms behind DGF and their impact on long-term graft function and survival. Since the main cause of DGF is ischemia and reperfusion injury, we use a functional definition of DGF, excluding other causes for DGF, like acute rejection, cyclosporin toxicity and surgical complications.

In chapter $\mathbf{2}$ we analyze the risk factors for DGF in a cohort of patients receiving a cadaveric transplant between 1983 and 1996, using this functional definition. Furthermore we study the impact of DGF on long-term events like graft function and graft loss.

Why some grafts develop DGF and others do not is unclear and needs to be elucidated. The mechanisms how DGF develops and therefore how DGF influences long-term graft faith also is unclear. To answer this question, there is need for a marker of functional renal mass that is easy assessable and can be repeated frequently.

In chapter $\mathbf{3}$ we use a parameter developed in the ${ }^{99 m}$ Technetium-mercaptoacetyltriglycine (MAG-3) renal scintigraphy, the Tubular Function Slope (TFS), as a marker of this functional renal mass. The differences in TFS between grafts reacting with DGF or not are analyzed, immediately after transplantation and during follow up.

The response of a graft on ischemic reperfusion injury depends on its protective and regenerative capacities. Some factors like donor age and donor gender are determinants of the functional renal mass and can not be manipulated. Others risk factors, however can. There is no doubt that cold ischemia time (CIT) and warm ischemia time (WIT) influence the occurrence of DGF and can be manipulated. Other factors like serum PTH levels and calcium levels and the use of calcium channel blockers (CCBs) are more controversial with regard to this aspect. However, the influence of serum calcium levels and the use of CCBs on the development of DGF has regained interest since many processes that take place during ischemia and reperfusion injury and subsequent processes like necrosis, apoptosis and cyclosporin toxicity, are thought to be directed by calcium dependent processes. Elucidating the role of serum calcium and PTH levels on the occurrence of DGF, may have clinical implications for the management of potential allograft recipients.

In Chapter 4 the impact of serum calcium levels and the use of CCBs on the development of DGF are analyzed. Serum calcium levels are not only correlated with the occurrence of DGF but also with the presence of calcium deposits in renal biopsies taken within the first week after transplantation. Furthermore the effect of CCBs is taken into consideration. 
In chapter $\mathbf{5}$ we describe a study in which the expression of protective enzymes is correlated with the occurrence of DGF and the presence of ATN in biopsies taken within the first week after transplantation.

Chapter 6 reviews the literature on DGF in renal transplantation.

Finally a summary is given in chapter 7 . 


\section{REFERENCES}

1. Bywaters EGL, Beall D. Crush injuries with impairment of renal function. British Medical Journal 1941:1: 427-432.

2. Lucke B. Lower nephron nephrosis (the renal lesions of the crush syndrome of burns, transfusion and other conditions aggecting the lower segments of the nephron). Military Surgery 1946:99.

3. Oliver J, MacDowell M, Tracey A. The pathogenesis of acute renal failure associated with traumatic and toxic injury. Renal ischemia, nephrotoxic damage and ischemicuric episode. J Clin Invest 1951:30.

4. Knochel JP. Rhabdomyolysis and Myoglobinuria. Annual Review of Medicine 1982:33:435-443.

5. Finn WF. Recovery from acute renal failure. In:Lazarus JM, Brenner BM, eds. Acute renal failure. New York: Churchill Livingstone, 1993:553.

6. Marcussen N, Lai R, Olsen S., Solez K. Morphometric and immunohistochemical investigation of renal biopsies from patients with transplant ATN, native ATN or acute graft rejection. Transplant Proc 1996:28: 470-476.

7. Donohoe JF, Venkatachalam MA, Bernard DB, Levinsky NG. Tubular leakage and obstruction after renal ischemia: structural-functional correlations. Kidney Int 1978:13:208-222.

8. Tanner GA, Steinhausen M. Tubular obstruction in ischemia-induced acute renal failure in the rat. Kidney Int Suppl 1976:6:S65-73.:S65-S73.

9. Finn WF, Chevalier RL. Recovery from postischemic acute renal failure in the rat. Kidney Int 1979:16: 113-123.

10. Baylis C, Rennke HR, Brenner BM. Mechanisms of the defect in glomerular ultrafiltration associated with gentamicin administration. Kidney Int 1977:12:344-353.

11. Stein JH, Gottschall J, Osgood RW, Ferris TF. Pathophysiology of a nephrotoxic model of acute renal failure. Kidney Int 1975:8: 27-41.

12. Myers BD, Moran SM. Hemodynamically mediated acute renal failure. N Engl J Med 1986:314:97-105.

13. Alejandro VS, Nelson WJ, Huie P et al. Postischemic injury, delayed function and Na+/K(+)-ATPase distribution in the transplanted kidney. Kidney Int 1995:48: 1308-1315.

14. Scandling JD, Myers BD. Pathophysiology of reduced glomerular filtration rate in delayed graft function. Curr Opin Nephrol Hypertens 1997:6: 405-409.

15. Thurau K, Boylan JW. Acute renal success. The unexpected logic of oliguria in acute renal failure. Am J Med 1976:61:308-315.

16. Boom H, Mallat MJ, De Fijter JW, Zwinderman AH, Paul LC. Delayed graft function influences renal function, but not survival. Kidney Int 2000:58: 859-866.

17. Brook NR, White SA, Waller JR, Veitch PS, Nicholson ML. Non-heart beating donor kidneys with delayed graft function have superior graft survival compared with conventional heart-beating donor kidneys that develop delayed graft function. Am J Transplant 2003:3: 614-618.

18. Koning $\mathrm{OHJ}$, Ploeg RJ, van Bockel JH et al. Risk factors for delayed graft function in cadaveric kidney transplantation - A prospective study of renal function and graft survival after preservation with University of Wisconsin solution in multi-organ donors. Transplantation 1997:63: 1620-1628.

19. Moreso F, Seron D, Gilvernet $\mathrm{S}$ et al. Donor age and delayed graft function as predictors of renal allograft survival in rejection-free patients. Nephrol Dialysis Transplant 1999:14: 930-935.

20. Ojo AO, Wolfe RA, Held PhJ, Port FK, Schmouder RL. Delayed graft function: risk factors and Implications for renal allograft survival. Transplantation 1997:63:968-974.

21. Sanchez-Fructuoso Al, Prats D, Torrente J et al. Renal transplantation from non-heart beating donors: $a$ 
promising alternative to enlarge the donor pool. J Am Soc Nephrol 2000:11:350-358.

22. Rosen S, Heyman SN. Difficulties in understanding human "acute tubular necrosis": limited data and flawed animal models. Kidney Int 2001:60: 1220-1224.

23. Shoskes DA, Halloran PF. Delayed graft function in renal transplantation: etiology, management and long-term significance. J Urol 1996:155:1831-1840.

24. Troppmann C, Gillingham KJ, Gruessner RWG et al. Delayed graft function in the absence of rejection has no long-term impact. Transplantation 1996:61:1331-1337.

25. Gjertson DW. A multi-factor analysis of kidney graft outcomes at one and five years posttransplantation: 1996 UNOS Update. Clin Transpl 1996:343-60:343-360.

26. Troppmann C, Gillingham KJ, Benedetti E et al. Delayed graft function, acute rejection and outcome after cadaveral renal transplantation. Transplantation 1995:59:962-968.

27. Lehtonen SRK, Isoniemi HM, Salmela KT, Taskinen El, Von willebrand EO, Ahonen JP. Long-term graft outcome is not necessarily affected by delayed onset of graft function and early acute rejection. Transplantation 1997:64: 103-107.

28. Giralclasse $M$, Hourmant $M$, Cantarovich $D$ et al. Delayed graft function of more than six days strongly decreases long-term survival of transplanted kidneys. Kidney Int 1998:54: 972-978.

29. Epstein M. Aging and the kidney. J Am Soc Nephrol 1996:7:1106-1122.

30. Nyengaard JR, Bendtsen TF. Glomerular number and size in relation to age, kidney weight, and body surface in normal man. Anat Rec 1992:232: 194-201.

31. Kappel B, Olsen S. Cortical interstitial tissue and sclerosed glomeruli in the normal human kidney, related to age and sex. A quantitative study. Virchows Arch A Pathol Anat Histol 1980:387:271-277.

32. Seron D, Carrera M, Grino JM et al. Relationship between donor renal interstitial surface and posttransplant function. Nephrol Dial Transplant 1993:8: 539-543.

33. Curschellas E, Landmann J, Durig M et al. Morphologic findings in "zero-hour" biopsies of renal transplants. Clin Nephrol 1991:36:215-222.

34. Darby JM, Stein K, Grenvik A, Stuart SA. Approach to management of the heartbeating 'brain dead' organ donor. JAMA 1989:261:2222-2228.

35. Es v.A., Hermans J., Bockel v.H.J., Persijn G.G., Hooff J.P., Graeff J.de. Effect of Warm Ischemia Time and HLA ( $A$ en $B$ ) matching on renal cadaveric graft survival and rejection episodes. Transplantation 1983:36: 255-258.

36. Daemen JW, Kootstra G, Wijnen RM, Yin M, Heineman E. Nonheart-beating donors: the Maastricht experience. Clin Transpl 1994::303-16.: 303-316.

37. Peters ThG., Shaver TR, Ames JE, Santiago-Delpin EA, Jones KW, Blanton JW. Cold ischemia and outcome in 17.937 cadaveric kidney transplants. Transpl Immunol 1995:59: 191-196.

38. Neumayer H.H., Eis M., Link J., Muhlberg J., Wagner K. Factors influencing Primary Kidney Graft Function. Transplant Proc 1986:XVIII: 1013-1117.

39. Schmidt R., Kupin W., Dumler F., Venkat K.K., Mozes M. Influence of the Pretransplant Hematocrit level on Early Graft Function in Primary Cadaveric Renal Transplantation. Transplantation 1993:55: 10341040.

40. Kahan B., Mickey R., Flechner S.M. et al. Multivariate Analysis of risk Factors Impacting on Immediate and Eventual Cadaver Allograft Survival in Cyclosporine-Treated Recipients. Transplantation 1987:43: 65-70.

41. Belli L.S., De Carlis L., Del Favero E. et al.The role of donor and recipient factors in initial renal graft nonfunction. Transplant Proc 1988:XX:861-864.

42. Pfaff WW, Howard RJ, Patton PR, Adams VR, Rosen CB, Reed AI. Delayed graft function after renal trans- 
plantation. Transplantation 1998:65:219-223.

43. Lieberthal W, Koh JS, Levine JS. Necrosis and apoptosis in acute renal failure. Semin Nephrol 1998:18: 505-518.

44. Ploeg R.J., Bockel v.H.J., Langendijk P.T.H. et al. Effect of preservation solution on results of cadaveric kidney transplantation. Lancet 1992:340: 129-137.

45. Parmar MS, Kjellstrand CM, Solez K, Halloran PF. Glomerular endothelial cell detachment in paired cadaver kidney transplants: evidence that some cadaver donors have pre-existing endothelial injury. Clin Transplant 1994:8: 120-127.

46. Feldman $\mathrm{HI}$, Fazio I, Roth $\mathrm{D}$ et al. Recipient body size and cadaveric renal allograft survival. J Am Soc Nephrol 1996:7: 151-157.

47. Traindl O, Langle F, Reading S et al. Secondary hyperparathyroidism and acute tubular necrosis following renal transplantation. Nephrol Dial Transplant 1993:8: 173-176.

48. Torregosa, Campistol, Fenollasa, Montesinos, Romar, Martinez de Osaba. Secondary Hyperparathyroidism and Post-Transplant Acute Tubular Necrosis. Nephron 1996:73:67-72.

49. Matas AJ, Gillingham KJ, Elick BA et al. Risk factors for prolonged hospitalization after kidney transplants. Clin Transplant 1997:11:259-264.

50. Koning $\mathrm{OHJ}$, Bockel van JH, Woude vd FJ, Persijn GG, Hermans J, Ploeg RJ. Risk factors for delayed graft function in University of Wisconsin solution preserved kidneys from multiorgan donors. Transplant Proc 1995:27: 752-753.

51. Neumayer HH, Kunzendorf $\mathrm{U}$, Schreiber M. Protective effects of calcium antagonists in human renal transplantation. Kidney Int Suppl 1992:36:S87-93.: S87-S93.

52. Solez K, Lilliane Morel-Maroger, Jean-Daniel Sraer. The Morphology of "Acute Tubular Necrosis" in man: Analysis of 57 Renal Biopsies and a comparison with the glycerol model. Medicine 1979:58:362-376.

53. Racusen L.C., Fivush B.A., Li Y.L., Slatnik I., Solez K. Dissociation of tubular cell detachement and tubular cell death in clnicial and experimental "acute tubular necrosis." Laboratory Investigation 1991:64: 546556.

54. Olsen S., Burdick J.F., Keown P.A., Wallace A.C., Racusen L.C., Solez K. Primary Acute Renal Failure ("Acute Tubular Necrosis") in the transplanted Kidney: Morphology and Pathogenesis. Medicine 1989: 173187.

55. van der Hoeven JA, Ploeg RJ, Postema F et al. Induction of organ dysfunction and up-regulation of inflammatory markers in the liver and kidneys of hypotensive brain dead rats: a model to study marginal organ donors. Transplantation 1999:68: 1884-1890.

56. Safirstein R, Price PM, Saggi SJ, Harris RC. Changes in gene expression after temporary renal ischemia. Kidney Int 1990:37: 1515-1521.

57. Toback F.G. Regeneration after acute tubular necrosis. Kidney Int 1992:41:226-246.

58. Harada A, Sekido N, Akahoshi T, Wada T, Mukaida N, Matsushima K. Essential involvement of interleukin8 (IL-8) in acute inflammation. J Leukoc Biol 1994:56:559-564.

59. Strieter RM, Koch AE, Antony VB, Fick RB, Jr., Standiford TJ, Kunkel SL. The immunopathology of chemotactic cytokines: the role of interleukin-8 and monocyte chemoattractant protein-1.J Lab Clin Med 1994:123: 183-197.

60. Haq M, Norman J, Saba SR, Ramirez G, Rabb H. Role of IL-1 in renal ischemic reperfusion injury. J Am Soc Nephrol 1998:9: 614-619.

61. Thervet E, Patey N, Legendre C, Noel LH, Kreis H. Prospective serial evaluation of cell adhesion molecule expression in transplanted kidneys. Transplant Proc 1995:27: 1007-1008.

62. Gaber L, Mann L, Hughes $C$ et al. Patterns of expression of endothelial leukocyte adhesion molecules 
upon revascularization of transplanted kidneys in correlation with allograft pathology. Transplant Proc 1995:27: 1003-1004.

63. Haug CE, Colvin RB, Delmonico FL et al. A phase I trial of immunosuppression with anti-ICAM-1 (CD54) $\mathrm{mAb}$ in renal allograft recipients. Transplantation 1993:55: 766-772.

64. Dragun D, Tullius SG, Park JK et al.ICAM-1 antisense oligodesoxynucleotides prevent reperfusion injury and enhance immediate graft function in renal transplantation. Kidney Int 1998:54:590-602.

65. Lieberthal W, Levine JS. Mechanisms of apoptosis and its potential role in renal tubular epithelial cell injury. Am J Physiol 1996:271:F477-F488.

66. Weinberg JM. The cell biology of ischemic renal injury. Kidney Int 1991:39:476-500.

67. Molitoris B.A. Ischemia-induced loss of epithelial polarity: potent role of the actin skeleton. Am J Physiol 1991:260: F769-F778.

68. Kribben A, Wieder ED, Wetzels JF et al. Evidence for role of cytosolic free calcium in hypoxia-induced proximal tubule injury. J Clin Invest 1994:93: 1922-1929.

69. Edelstein CL, Ling H., Schrier RW. The Nature of renal cell injury. Kidney Int 1997:51:1341-1351.

70. Yu L, Gengaro PE, Niederberger M, Burke TJ, Schrier RW. Nitric oxide: a mediator in rat tubular hypoxia/ reoxygenation injury. Proc Natl Acad Sci U S A 1994:91: 1691-1695.

71. Noiri E, Peresleni T, Miller F, Goligorsky MS. In vivo targeting of inducible NO synthase with oligodeoxynucleotides protects rat kidney against ischemia. J Clin Invest 1996:97: 2377-2383.

72. Granger DN. Role of xanthine oxidase and granulocytes in ischemia-reperfusion injury. Am J Physiol 1988:255: H1269-H1275.

73. Wilhelm SM, Simonson MS, Robinson AV, Stowe NT, Schulak JA. Endothelin up-regulation and localization following renal ischemia and reperfusion. Kidney Int 1999:55: 1011-1018.

74. Yamada K, Gunji Y, Hishikawa E et al. Possible involvement of endothelin in posttransplant acute tubular necrosis. I: Studies in renal transplant patients. Transplantation 1994:57: 1137-1139.

75. Shoskes DA, Xie Y, Gonzalez-Cadavid NF. Nitric oxide synthase activity in renal ischemia-reperfusion injury in the rat: implications for renal transplantation. Transplantation 1997:63:495-500.

76. Cheeseman K.H., Slater T.F. An introduction to free radical biochemistry. Br Med Bull 1993:49:481-493.

77. Granger DN, Korthuis RJ. Physiologic mechanisms of postischemic tissue injury. [Review]. Annu Rev Physiol 1995:57:311-332.

78. Zimmerman BJ, Granger DN. Mechanisms of reperfusion injury. Am J Med Sci 1994:307:284-292.

79. Baud L, Ardaillou R. Involvement of reactive oxygen species in kidney damage. Br Med Bull 1993:49: 621-629.

80. Molitoris BA, Leiser J, Wagner MC. Role of the actin cytoskeleton in ischemia-induced cell injury and repair. Pediatr Nephrol 1997:11:761-767.

81. Morita K, Seki T, Nonomura K, Koyanagi T, Yoshioka M, Saito H. Changes in renal blood flow in response to sympathomimetics in the rat transplanted and denervated kidney. Int J Urol 1999:6:24-32.

82. Singh I, Gulati S, Orak JK, Singh AK. Expression of antioxidant enzymes in rat kidney during ischemiareperfusion injury. Mol Cell Biochem 1993:125: 97-104.

83. Pollak R., Andrisevic JH, Maddux MS, Gruber SA, Paller MS. A randomized double-blind trial of the use of human recombinant superoxide dismutase in renal transplantation. Transplantation 1993:55: 57-60.

84. Schneeberger $H$, Illner WD, Abendroth $D$ et al. First clinical experiences with superoxide dismutase in kidney transplantation--results of a double-blind randomized study. Transplant Proc 1989:21: 12451246.

85. Land W. The potential Impact of the reperfusion Injury on acute and chronic Rejection events following organ transplantation. Transplant Proc 1994:26:3169-3171. 
86. Grone HJ, Weber K, Grone E, Helmchen U, Osborn M. Coexpression of keratin and vimentin in damaged and regenerating tubular epithelia of the kidney. Am J Pathol 1987:129:1-8.

87. Wallin A, Zhang G, Jones TW, Jaken S, Stevens JL. Mechanism of the nephrogenic repair response. Studies on proliferation and vimentin expression after 35S-1,2-dichlorovinyl-L-cysteine nephrotoxicity in vivo and in cultured proximal tubule epithelial cells. Lab Invest 1992:66:474-484.

88. Witzgall R, Brown D, Schwarz C, Bonventre JV. Localization of proliferating cell nuclear antigen, vimentin, c-Fos, and clusterin in the postischemic kidney. Evidence for a heterogenous genetic response among nephron segments, and a large pool of mitotically active and dedifferentiated cells. J Clin Invest 1994:93:2175-2188.

89. Miller SB, Martin DR, Kissane J, Hammerman MR. Rat models for clinical use of insulin-like growth factor I in acute renal failure. Am J Physiol 1994:266: F949-F956.

90. Wang S., Hirschberg R. Role of growth factors in acute renal failure. Nephrol Dial Transplant 1997:12: 1560-1563.

91. Verstrepen WA, Nouwen EJ, Yue XS, De Broe ME. Altered growth factor expression during toxic proximal tubular necrosis and regeneration. Kidney Int 1993:43:1267-1279.

92. Humes HD, Cieslinski DA, Coimbra TM, Messana JM, Galvao C. Epidermal growth factor enhances renal tubule cell regeneration and repair and accelerates the recovery of renal function in postischemic acute renal failure. J Clin Invest 1989:84: 1757-1761.

93. Kawaida K, Matsumoto K, Shimazu H, Nakamura T. Hepatocyte growth factor prevents acute renal failure and accelerates renal regeneration in mice. Proc Natl Acad Sci U S A 1994:91:4357-4361.

94. Franklin SC, Moulton M, Sicard GA, Hammerman MR, Miller SB. Insulin-like growth factor I preserves renal function postoperatively. Am J Physiol 1997:272:F257-F259.

95. Safirstein R. Gene expression in nephrotoxic and ischemic acute renal failure.J Am Soc Nephrol 1994:4: 1387-1395.

96. Thadhani R., Pascual M., Bonventre J.V. Acute Renal Failure. New England Journal of Medicine 1996:334: 1448-1459.Fig. 1 


\section{DELAYED GRAFT FUNCTION INFLUENCES RENAL FUNCTION, BUT NOT SURVIVAL}

Henk Boom, Marko J.K. Mallat, Johan W. de Fijter, Aeilko H. Zwinderman, Leendert C. Paul

Kidney International, 2000;58: 859- 866 
Background In renal transplantation, the impact of delayed graft function (DGF) on prognosis is controversial. We analyzed the risk factors of DGF and its impact on graft function and prognosis.

Methods 734 cadaveric renal transplants performed between 1983 and 1997 were analyzed. DGF was diagnosed when serum creatinine levels increased, remained unchanged or decreased less than $10 \%$ per day in three consecutive days, in the first week after transplantation. Creatinine clearances of more or less than $50 \mathrm{ml} / \mathrm{min}$ or $30 \mathrm{ml} / \mathrm{min}$ at 1 year were used as cut-off points for optimal and suboptimal graft function, respectively. The logistic regression model was used to identify independent risk factor related to DGF and renal function 1 year after transplantation. The Cox regression model was used to examine the influence of DGF on long-term graft survival.

Results Multivariate analysis revealed the following risk factors for DGF (Odds Ratio, 95\% Confident Interval): recipient pre-transplantation mean arterial blood pressure of less than $100 \mathrm{mmHg}: 2.08$ (1.43 -3.03), female donor to male recipient combination: 1.55, $1.02-2.35$, donor age of more than 50 years: $2.21,1.49-3.26$, cold ischemia time of more than 28 hours: $1.78,1.19-2.63$ and peak panel reactive antibodies of more than $50 \%: 1.7,1.15-2.55$. The incidence of DGF was one of the independent risk factors for suboptimal graft function at 1 year: $1.68,1.14-2.48$ together with donor age of more than 50 years: $2.39,1.61-3.57$, female donor gender: $1.99,1.42-2.78$, the occurrence of acute rejection episodes $2.66,1.87$ -3.78, peak panel reactive antibodies of more than $50 \%: 1.67,1.15-2.47$ and sharing of 1-3 vs. 4-8 CREGs 1.65, 1.09-2.49. Moreover, DGF was one of the two independent risk factors for acute rejection episodes, but it had no independent effect on graft survival.

Conclusion Several risk factors for DGF were identified of which a low recipient pre-transplant mean arterial blood pressure, the transplantation of kidneys from female donors to male recipients and a prolonged cold ischemia time are potentially avoidable. Although DGF is one of the several risk factors of acute rejection and suboptimal function at 1 year, it is not independently associated with an increased rate of graft loss. 


\section{INTRODUCTION}

In renal transplantation there is controversy regarding the impact of delayed graft function (DGF) on long-term outcome. This may relate to different criteria used to define DGF or to differences in data analysis. Most authors use the need for dialysis within the first week as the diagnostic inclusion criterion but this does not differentiate the various causes of DGF such as ischemia- reperfusion injury or early acute rejection episodes. In addition, the degree of renal damage is often not taken into consideration. In the UNOS registry, DGF defined as the need for dialysis in the first week after transplantation had a significant and independent impact on graft half-life. This effect was distinct from cold ischemia time, occurrence of acute rejection episodes, donor age and serum creatinine levels $(1,2)$. Others found a detrimental effect of DGF, also defined as the need for dialysis in the first week, on graft survival only when it was complicated by one or more acute rejection episodes $(3,4)$. Using the time required to reach a Cockroft renal clearance of more than $10 \mathrm{ml} / \mathrm{min}$, DGF lasting for more than 6 days had a deleterious effect on graft survival whereas DGF of shorter duration did not influence graft survival (5). In the present paper, we analyzed the risk factors of DGF defined by stringent criteria, independent from the need of dialysis. Moreover, as graft function at 1 year is a strong surrogate marker of late graft outcome $(6,7)$, we also studied the impact of DGF on 1-year graft function, graft loss and long-term prognosis.

\section{MATERIALS EN METHODS}

\section{Patients}

All patients who received a cadaveric renal transplant in our center between April of 1983 and December of 1996 were included in the study. Kidneys were allocated according to the matching and allocation criteria of Eurotransplant. We aimed to accept kidneys with no more than two HLA-mismatches with a priority for HLA-DR matching.

\section{Immunosuppressive regimen}

The standard immunosuppressive regimen consisted of prednisone and Cyclosporine-A [Sandimmune (CsA)]. Sixty-two patients $(8,4 \%)$ did not receive CsA and were initially treated with azathioprine (Aza) in a dosage of $2 \mathrm{mg} / \mathrm{kg} /$ day. Two hundred and seven (28\%) patients initially treated with CsA were randomly or on clinical grounds converted to Aza within the first six post-transplant months. CsA was administered intravenously in a dose of $3 \mathrm{mg} / \mathrm{kg} /$ day for the first 48 hours, starting at the onset of surgery. The initial oral dose of CsA was $10 \mathrm{mg} / \mathrm{kg} /$ day from day 2 onward, divided in three daily doses and subsequently tapered. Doses were adjusted according to CsA trough levels. After 6 weeks, the total dose was given as a once daily dose. In the first three months, the target 24-hour CsA trough level was between 250 and $500 \mu \mathrm{g} / \mathrm{l}$. We targeted to a 24-hour trough level range after 3 months between 50 and $150 \mu \mathrm{g} / \mathrm{l}$. All patients received $20 \mathrm{mg}$ of prednisone starting on day one; 
this dose was reduced by $2.5 \mathrm{mg}$ every fortnight until a daily maintenance dose of $10 \mathrm{mg}$ was reached. Rejection episodes were treated with $1 \mathrm{gram}$ of methylprednisolone intravenously for 3 days or rabbit anti-thymocyte globulin for 10 days, as previously described (8).

\section{Definitions}

To exclude patients who were dialyzed for reasons other than impaired graft function, we diagnosed delayed graft function (DGF) retrospectively if the serum creatinine level increased, remained unchanged or decreased by less than $10 \%$ per day immediately after surgery during three consecutive days for more than 1 week. If a graft biopsy taken within the first post-transplant week showed rejection, it was assumed that the graft did not have DGF and it was categorized as primary function. Primary Non-Function (PNF) was defined as the absence of a decrease in the serum creatinine level that ultimately resulted in graft nephrectomy. Primary Function (PF) was defined as a decrease of the serum creatinine level of more than $10 \%$ per day over three consecutive days within the first week after surgery.

Graft loss was defined as resumption of dialysis treatments. Early graft loss was defined as graft loss within the first year after transplantation. Graft survival was censored for patient death with functioning graft. Renal Function at one year was calculated using the CockroftGault Formula (9):

Creatinine clearance $=((140$-age $) \times$ weight $(\mathrm{kg}) \times A) /($ Serum creatinine $(\mu \mathrm{mol} / \mathrm{l}) \times 0,8))$ In which $A=1$ in males and $A=0,85$ in females.

\section{Study Design}

Risk factors of DGF and the impact of DGF on renal function within the first year were analyzed and compared with grafts experiencing PF. Moreover, a broad spectrum of donor, recipient- and transplantation related variables were studied (Table 1). Acute rejection episodes were diagnosed on clinical grounds and confirmed by biopsy, unless a biopsy could not be obtained. Rejections were classified as predominantly interstitial or vascular, although most vascular rejections had variable degrees of interstitial inflammation. Mean arterial blood pressure (MAP) was calculated, using the following formula:

$$
\text { MAP }=(\text { Diastolic Blood Pressure } \times 2+\text { Systolic Blood Pressure }) / 3
$$

Cross-Reactive Groups (GREG) are defined as the HLA public epitopes of the class-I MHCantigens, based on the amino acid residue system as proposed for UNOS allocation (10). Not only the degree of mismatching, but also the effect of sharing between donor and recipient of HLA antigens was studied. The term "mismatch" was used for the number of HLA antigens that donor and recipient did not have in common, whereas the term "shares" was used for the number of corresponding HLA antigens between donor and recipient.

In our study population, the mean endogenous creatinine clearance at 1 year is approximately $50 \mathrm{ml} / \mathrm{min}$ (fig.1). Arithmetical graft half-life is 70 years for grafts with a creatinine clearance of more than $50 \mathrm{ml} / \mathrm{min}$ and 18,5 years for grafts with a 1-year creatinine clearance of less than $50 \mathrm{ml} / \mathrm{min}$. Therefore, patients having a creatinine clearance of more or 
less than $50 \mathrm{ml} / \mathrm{min}$ were categorized as optimal or suboptimal function respectively. We furthermore analyzed the data using a graft function of more or less than $30 \mathrm{ml} / \mathrm{min}$ as the dependent variable. This cut-off point represents the mean minus one standard deviation and is a more stringent outcome parameter. Arithmetical graft half-life is 53 years for grafts with a creatinine clearance of more than $30 \mathrm{ml} / \mathrm{min}$ and 7 years for grafts with a 1-year creatinine clearance of less than $30 \mathrm{ml} / \mathrm{min}$. To predict outcome at 1 year, patients experiencing graft-loss within this year, were categorized as having suboptimal function at 1 year. To study the additional impact of DGF on outcome after the first year, we analyzed its effect in different strata of renal function after 1 year.

Fig. 1 Frequency-distribution curve of the Cockroft clearances at 1 year in 604 transplant patients.

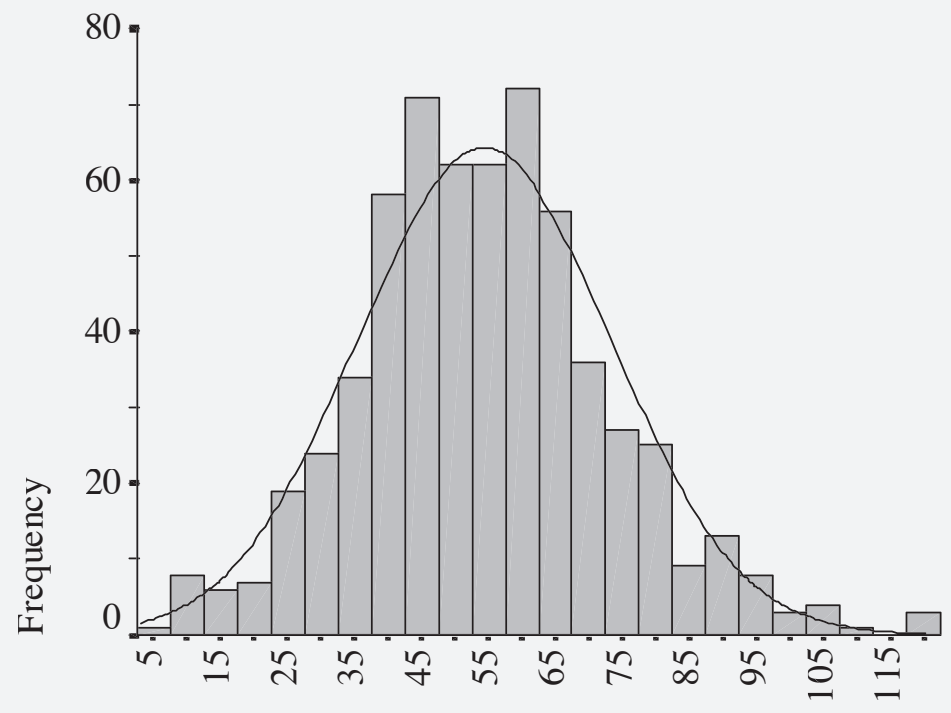

Cockroft clearance $(\mathrm{ml} / \mathrm{min})$

\section{Statistical analysis}

The logistic regression model was used to determine the factors significantly related to DGF, early graft loss, acute rejection and renal function at one year in an uni-variate way. The significant predictors of each parameter of renal function were next fitted in a multivariate model. Step forward selection techniques were used to determine significant risk factors. The risk is expressed as Odds Ratio (OR) $+95 \%$ Confidence Interval $(95 \% \mathrm{Cl})$. The impact of a suboptimal Cockroft clearance at 1 year on late graft loss was studied using the Cox regression model. By using this model we were able to correct for the time of follow up to graft loss. The risk is expressed as a Relative Risk (RR) $+95 \%$ Confidence Interval $(95 \% \mathrm{Cl})$. We used the Kaplan Meier survival analysis (Log-rank test) to compare graft failure in the different strata of Cockroft clearance at 1 year. We used the SPSS software package (9.0) for all analyses. 


\section{RESULTS}

Seven hundred and ninety patients were included in the study; 24 (3.0\%) were not analyzed because of primary non-function and 32 (4.1\%) because of missing data on DGF. Demographic data are shown in Table 1. DGF was diagnosed if the serum creatinine level increased, remained unchanged or decreased less than $10 \%$ per day immediately after surgery during three consecutive days for more than 1 week. Twenty eight $(11.8 \%)$ of the patients experiencing renal dysfunction in the first week, making dialysis treatment necessary, had a biopsy proven acute rejection episode and were classified as PF.

Table 1: Characteristics at time of transplantation.

\begin{tabular}{|c|c|c|c|}
\hline Risk Factor & $\begin{array}{l}\text { Total } \\
(\mathrm{N}=734)\end{array}$ & $\begin{array}{l}\text { PF } \\
N=551 \\
(75.1 \%)\end{array}$ & $\begin{array}{l}\text { DGF } \\
\mathrm{N}=183 \\
(24.9 \%)\end{array}$ \\
\hline \multicolumn{4}{|l|}{ Recipient } \\
\hline Age (years) & $46(13)$ & $46(12)$ & $47(14)$ \\
\hline Gender (\% female) & 38 & 38 & 39 \\
\hline Peak panel reactive antibodies (PRAH) (\%) & $31(32)$ & $29(31)$ & $36(35)$ \\
\hline Current panel reactive antibodies (PRAC) (\%) & $12(23)$ & $11(22)$ & $14(26)$ \\
\hline MAP before transplantation $(\mathrm{mmHg})$ & $109(16)$ & $110(17)$ & $106(16)$ \\
\hline \multicolumn{4}{|l|}{ Donor } \\
\hline Age (yrs.) & $37(14)$ & $36(14)$ & $42(14)$ \\
\hline Gender (\% female) & 41.7 & 44.9 & 40.5 \\
\hline \multicolumn{4}{|l|}{ Cause of death: } \\
\hline Trauma / Cardio-vascular (\%) & $47.5 / 52.5$ & $49.5 / 50.5$ & $41.8 / 58.2$ \\
\hline \multicolumn{4}{|l|}{ Transplantation related } \\
\hline \multicolumn{4}{|l|}{ Gender Mismatch } \\
\hline No mismatch (\%) & 54 & 56 & 46 \\
\hline Donor male-Recipient female (\%) & 21 & 20 & 24 \\
\hline Donor female-Recipient male (\%) & 25 & 23 & 30 \\
\hline \multicolumn{4}{|l|}{ Transplant status } \\
\hline First transplant (\%) & 83 & 76 & 79 \\
\hline$>1$ transplant $(\%)$ & 17 & 24 & 21 \\
\hline Cold Ischemia Time (hours) & $29(7)$ & $28(7)$ & $30(7)$ \\
\hline Warm Ischemia Time (min.) & $28(9)$ & $28(9)$ & $28(9)$ \\
\hline \multicolumn{4}{|l|}{ Immuno-suppression at transplantation } \\
\hline Aza/pred. (\%) & 8 & 9 & 6 \\
\hline \multicolumn{4}{|l|}{ Immuno-suppression at 6 months } \\
\hline Aza/pred. (\%) & 28.1 & 29.6 & 23.5 \\
\hline \multicolumn{4}{|l|}{ HLA } \\
\hline Mismatch & $1.9(1.1)$ & $1.9(1.1)$ & $1.9(1.2)$ \\
\hline Shares & $3.7(1.0)$ & $3.6(1.0)$ & $3.6(1.1)$ \\
\hline
\end{tabular}




$\begin{array}{llll}\text { GREG } & & & \\ \quad \text { Mismatch } & 1.2(1.1) & 1.2(1.1) & 1.1(1.0) \\ \quad \text { Shares } & 4.5(1.2) & 4.5(1.2) & 4.5(1.1) \\ \text { Number of rejection episodes < 1 year } & & & \\ \quad 1(\%) & 23 & 23 & 24 \\ 2(\%) & 23 & 20 & 30 \\ >2(\%) & 11 & 10 & 13 \\ \text { Type of rejection < 1 year } & & & \\ \text { Interstitial (\%) } & 36 & 34 & 41 \\ \quad \text { Vascular (\%) } & 14 & 12 & 21 \\ \text { Clinical (\%) } & 8 & 8 & 7 \\ \text { Graft Loss within 1 year (\%) } & 13 & 11 & 19 \\ \text { Clearance at 1 year (ml/min) } & 53(20) & 55(20) & 47(21)\end{array}$

Data are expressed as mean \pm SD unless otherwise stated

\section{Risk Factors for Delayed Graft Function}

In an univariate analysis, donor age of more than 50 years, mean arterial blood pressure (MAP) of less than $100 \mathrm{mmHg}$, cold ischemia time (CIT) of over 28 hours, transplantation of a kidney from a female donor to a male recipient and peak panel reactive antibodies of over $50 \%$ were associated with DGF. All these factors were subsequently entered in a multivariate analysis and remained significant (table 2).

\section{Risk factors for sub-optimal graft function after one year}

To analyze the impact of DGF and other factors on graft function after 1 year we used the creatinine clearance of more or less than $50 \mathrm{ml} / \mathrm{min}$ as the dependent variable. The univariate analysis revealed DGF as a risk factor for a sub-optimal graft function after 1 year. Other risk factors for suboptimal function included donor age of more than 50 years, female donor gender, donor cause of death (cardio-vascular versus trauma), total warm ischemia time, peak panel reactive antibodies of more than $50 \%$, current panel reactive antibodies, sharing of less than 3 cross reactive antigens groups (CREG) and the number of acute rejection episodes within the first year. All these factors were entered in a multivariate analysis and as shown in table 3, remained significant with the exception of donor cause of death and the warm ischemia time.

As a creatinine clearance of $30 \mathrm{ml} / \mathrm{min}$ is a more stringent outcome variable for graft function, we also analyzed $30 \mathrm{ml} / \mathrm{min}$ at 1 year as the dependent variable. In the uni-variate analysis, DGF remained a significant risk factor, as were donor age of more than 50 years, female donor gender and sharing of 3 or less CREGs. The use of an initial Aza-based immunosuppressive regimen, the occurrence of acute rejection episodes and vascular rejection, were all associated with suboptimal outcome. Table 4 shows the results of the multivariate analysis. The incidence of DGF (OR $1.81 ; 95 \% \mathrm{Cl} 1.17-2.81)$, the use of kidneys from donors older than 50 years (OR $2.11 ; 95 \% \mathrm{Cl} 1.35$ - 3.29), the initial use of an Aza-based immunosuppressive regimen (OR 2.53;95\% Cl $1.32-4.83$ ), the sharing of 3 or less CREGs (OR 2.53;95\% Cl 1,30 
$-3,35$ ) and the incidence of acute rejection episodes (OR 4.00; 95\% $\mathrm{Cl} 2.41-5.65$ ) remained significantly and independently related to a graft function of less than $30 \mathrm{ml} / \mathrm{min}$ after 1 year. We were not able to analyze recipient's age, weight and gender as risk factors, because these variables were used in the Cockroft-Gault method to estimate graft function.

Table 2: Risk factors for Delayed Graft Function ${ }^{a}$

\begin{tabular}{|c|c|c|}
\hline Variable & Odds Ratio & $95 \% \mathrm{Cl}^{b}$ \\
\hline \multicolumn{3}{|l|}{ Donor age } \\
\hline$>50$ years & 2.21 & $1.49-3.26$ \\
\hline \multicolumn{3}{|c|}{ Recipient MAP before transplantation } \\
\hline$<100 \mathrm{mmHg}$ & 2.08 & $1.43-3.03$ \\
\hline \multicolumn{3}{|l|}{ Cold Ischemia Time } \\
\hline$>28$ hours & 1.78 & $1.19-2.63$ \\
\hline \multicolumn{3}{|l|}{ Gender Mismatch } \\
\hline No mismatch & 1 & \\
\hline Donor male- Recipient female & 1.09 & $0.69-1.73$ \\
\hline Donor female- Recipient male & 1.55 & $1.02-2.35$ \\
\hline \multicolumn{3}{|l|}{ Peak Panel Reactive Antibodies } \\
\hline$>50 \%$ & 1.7 & $1.15-2.55$ \\
\hline
\end{tabular}

${ }^{a}$ Multivariate analysis ${ }^{b} 95 \% \mathrm{Cl}: 95 \%$ Confidence Interval

Table 3: Risk factors for suboptimal function (creatinine clearance $<50-\mathrm{ml} / \mathrm{min}$ ) at 1 year after transplantation, including graft-loss in the first year ${ }^{a}$

\begin{tabular}{lll}
\hline Variable & Odds Ratio & $\mathbf{9 5 \%} \mathbf{C l}^{b}$ \\
\hline $\begin{array}{l}\text { Delayed graft function } \\
\text { Donor age }\end{array}$ & 1.68 & $1.14-2.48$ \\
$\quad>50$ years & 2.39 & $1.61-3.57$ \\
$\begin{array}{l}\text { CREG-sharing } \\
\quad 1-3 \text { shares vs. 4-8 shares }\end{array}$ & 1.65 & $1.09-2.49$ \\
$\begin{array}{l}\text { Number of acute rejection episodes } \\
>1\end{array}$ & 2.66 & $1.87-3.78$ \\
$\begin{array}{l}\text { Donor Gender } \\
\text { Female vs. male }\end{array}$ & 1.99 & $1.42-2.78$ \\
$\begin{array}{l}\text { Peak panel reactive antibodies } \\
>50 \%\end{array}$ & 1.67 & $1.15-2.47$ \\
\hline
\end{tabular}

${ }^{a}$ Multivariate analysis; ${ }^{b}$ 95\% Cl: $95 \%$ Confidence Interval 
Delayed graft function influences renal function, but not survival

Table 4: Risk Factors for a 1 -year creatinine clearance $<30 \mathrm{ml} / \mathrm{min}$ including graft-loss within 1 year ${ }^{a}$

\begin{tabular}{lll}
\hline Variable & Odds Ratio & $\mathbf{9 5 \% \mathbf { C l } ^ { b }}$ \\
\hline $\begin{array}{l}\text { Delayed graft function } \\
\text { Donor Age }\end{array}$ & 1.81 & $1.17-2.81$ \\
$\quad>50$ years & 2.11 & $1.35-3.29$ \\
$\begin{array}{l}\text { Immuno-suppressive regimen at time of transplan- } \\
\text { tation }\end{array}$ & \\
$\quad \begin{array}{l}\text { Aza/Pred.Vs. CsA/Pred. } \\
\text { CREG- sharing } \\
\quad 1-3 \text { vs. 4-8 shares }\end{array}$ & 2.53 & $1.32-4.83$ \\
$\begin{array}{l}\text { Number of acute rejection episodes } \\
>1\end{array}$ & 2.53 & $1.30-3.35$ \\
\hline
\end{tabular}

${ }^{a}$ Multivariate analysis; ${ }^{b}$ 95\% Cl: $95 \%$ Confidence Interval

Occurrence of acute rejection episodes within one year after transplantation.

DGF was associated with an increasing likelihood of acute rejection episodes in an univariate analysis as were female donor gender, HLA-DR mismatch, peak panel reactive antibodies of more than $50 \%$ and retransplant status of the recipient. HLA-sharing correlates inversely with the incidence of acute rejection episodes. Table 5 shows the independent risk factors for acute rejection in the first year, in the multi-variate analysis. The incidence of acute rejection episodes was independently associated with DGF (OR 1.61; $95 \% \mathrm{Cl} 1.11-$ 2.33), an increase of HLA-DR mismatch (OR 2.36; $95 \% \mathrm{Cl} 1.68-3.31)$ and peak panel reactive antibodies of more than $50 \%$ (OR 1.60; $95 \% \mathrm{Cl} 1.12-2.30)$.

Table 5: Riskfactors for the occurrence of acute rejection episodes within 1 year ${ }^{a}$

\begin{tabular}{lll}
\hline Variable & Odds Ratio & $\mathbf{9 5 \% \mathbf { C l } ^ { b }}$ \\
\hline $\begin{array}{l}\text { Delayed Graft Function } \\
\text { Mismatch HLA DR } \\
\quad>=1\end{array}$ & 1.61 & $1.11-2.33$ \\
$\begin{array}{l}\text { Peak Panel Reactive Antibodies } \\
\quad>50 \%\end{array}$ & 2.36 & $1.68-3.31$ \\
\hline
\end{tabular}

${ }^{a}$ Multivariate analysis; ${ }^{b}$ 95\% Cl: $95 \%$ Confidence Interval

\section{Influence of DGF on graft loss}

Fig. 2 shows the univariate Kaplan Meier graft survival estimates, of patients with primary function and patients with delayed graft function. There is a significantly decreased graftsurvival in patients with DGF, with an arithmetical graft half-life of 12.8 years, compared to 21.7 years for patients not experiencing DGF. The main effect of DGF on graft loss seems to take place in the first year, whereas after the first year, especially after 6 years there is no dif- 
ference in outcome (data not shown). The short-and long-term graft losses were analyzed separately.

In an univariate analysis, DGF was correlated with graft loss within the first year, as were female donor gender, an Aza-based immunosuppressive regimen, CIT of more than 24 hours and the number and type of rejection episodes. Sharing of HLA Class- 1 antigens correlated inversely with graft loss. However, when the data were entered in a multivariate analysis neither DGF (OR 1.52; 95\% Cl $0.92-2.53$ ) nor cold ischemic time (OR 1.17; $95 \% \mathrm{Cl} 0.72-1.88$ ) remained a risk factor for graft-loss within the first year. Acute rejection episodes, especially vascular rejection (OR 9.32; 95\% Cl 4.77 - 18.2), female donor gender (OR 1.70; 95\% Cl 1.07 - 2.68), and an Aza-based immunosuppressive regimen (OR $2.07 ; 95 \% \mathrm{Cl} 1.05-4.09)$ remained independently associated with graft loss within the first year (Table 6).

Graft loss after the first year was associated in a univariate analysis with recipient age of less than 50 years and donor age of more than 50 years, the occurrence of acute rejection episodes in the first year and a cold ischemia time of more than 34 hours. Increased sharing of HLA antigens, sharing of 4-8 vs. 3 or less CREGs and higher creatinine clearance at 1 year correlated inversely with graft loss. DGF was not an independent risk factor for graft loss after the first year (OR $1.58 ; 95 \% \mathrm{Cl} 0.98-2.54$ ). Table 7 shows the results of the multivariate analysis. The occurrence of acute rejection episodes (OR 1.38; $95 \% \mathrm{Cl} 1.11$ 1.71), recipient age of less than 50 years (OR 1.70; $95 \% \mathrm{Cl} 1.00-2.86)$ and a cold ischemia time of more than 34 hours (OR 1.90;95\% Cl 1.20 - 3.05) were all independent risk factors for late graft loss. As soon as the Cockroft clearance after 1 year was fitted in the model as a continuous parameter, CIT and recipient age were no risk factors anymore. Therefore, graft function at 1 year was a strong predictor of late graft outcome (RR 0.96; 95\% Cl 0.95$0.97 \mathrm{per} \mathrm{ml} / \mathrm{min}$ ). When graft function after 1 year was divided in 4 strata of clearance of $>50 \mathrm{ml} / \mathrm{min}$, clearance of $40-50 \mathrm{ml} / \mathrm{min}$, clearance of 30-40 ml$/ \mathrm{min}$ and clearance of $<30$ $\mathrm{ml} / \mathrm{min}$, DGF had no additional effect on graft survival in any stratum (fig.3).

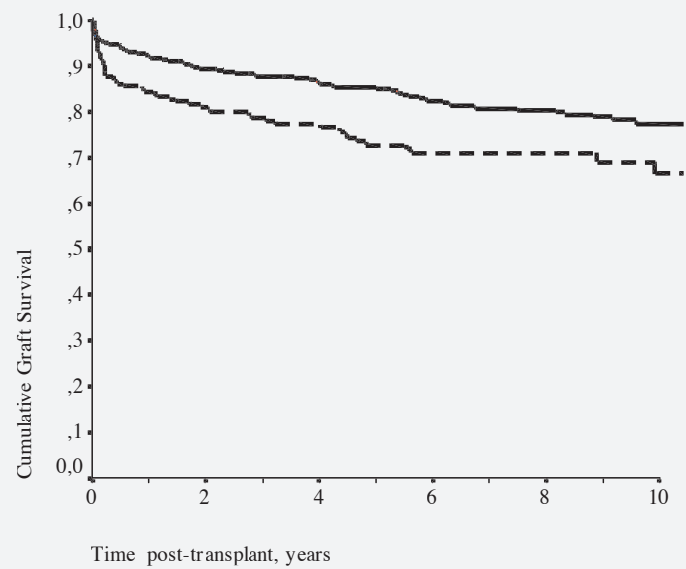

Fig. 2 Graft survival according to the incidence of DGF.

Kaplan-Meier estimates for transplants experiencing PF (solid rule; $\mathrm{N}=550$ ); half-life: 21.7 years and experiencing DGF (dashed rule; $\mathrm{N}=183$ ); arithmetical half-life: 12.8 years. Logrank test $P=0,0005$. 
Table 6: Risk factors for graft loss within 1 year ${ }^{a}$

\begin{tabular}{lll}
\hline Variable & Odds Ratio & $\mathbf{9 5 \%} \mathbf{C l}^{b}$ \\
\hline $\begin{array}{l}\text { Donor Related } \\
\text { Gender of donor }\end{array}$ & 1 & \\
$\quad \begin{array}{l}\text { Female vs. Male } \\
\text { Transplantation related }\end{array}$ & 1.70 & $1.07-2.68$ \\
$\quad$ Immunosuppressive Regimen & & \\
$\quad$ Aza / Pred. vs. CsA / Pred. & 2.07 & $1.05-4.09$ \\
$\quad$ Type of rejection < 1 year & & \\
$\quad$ No & 1 & \\
$\quad$ Interstitial & 2.64 & $1.33-5.22$ \\
$\quad$ Vascular & 9.32 & $4.77-18.2$ \\
$\quad$ Clinical (no biopsy) & 3.61 & $1.45-8.99$ \\
\hline
\end{tabular}

${ }^{a}$ Multivariate analysis; ${ }^{b} 95 \% \mathrm{Cl}: 95 \%$ Confidence Interval

Table 7: Riskfactors of graft loss after 1 year $^{a}$

\begin{tabular}{lll}
\hline Variable & Relative Risk & $\mathbf{9 5 \%} \mathbf{C l}^{b}$ \\
\hline $\begin{array}{l}\text { Recipient age } \\
\quad<50 \text { years }\end{array}$ & 1.70 & $1.00-2.86$ \\
$\quad$ Cold Ischemia Time & & \\
$\quad>34$ hours & 1.91 & $1.20-3.05$ \\
Occurrence of acute rejection episodes & 1,38 & $1.11-1.71$ \\
\hline
\end{tabular}

${ }^{a}$ Multivariate analysis; ${ }^{b} 95 \% \mathrm{Cl}: 95 \%$ Confidence Interval 
Fig. 3 Graft survival according to graft function 1 year after transplantation.

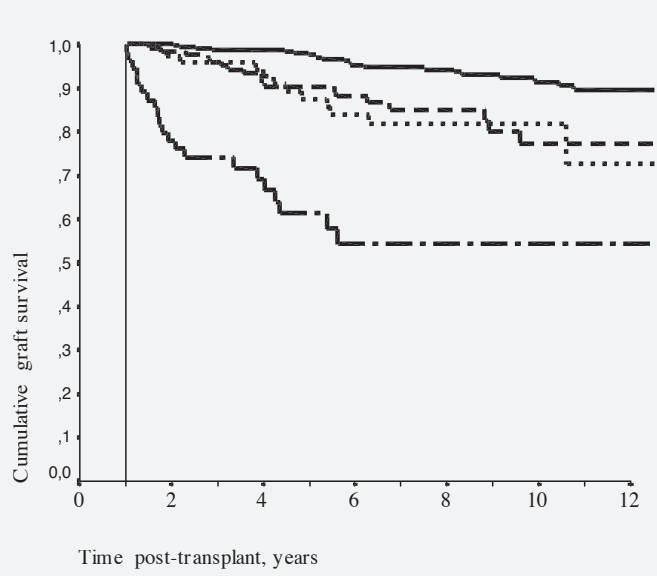

Kaplan-Meier estimates for transplants experiencing a 1-year creatinine clearance of $>50 \mathrm{ml} / \mathrm{min}$ (solid rule; $\mathrm{N}=339$ ); arithmetical half-life: 70 years; $40-50 \mathrm{ml} / \mathrm{min}$ (short dashed rule $\mathrm{N}=135$ ); arithmetical half-life: 30 years; $30-40$ $\mathrm{ml} / \mathrm{min}$ (long dashed rule; $\mathrm{N}=79$ ) arithmetical half-life: 25 years and < $30 \mathrm{ml} / \mathrm{min}$. (long, short, long dashed rule; $\mathrm{N}=56$ ), half-life: 7 years

\section{DISCUSSION}

In this retrospective study we examined the risk factors and prognostic significance of DGF in renal transplantation. In contrast to most other studies that examined this, we used a more stringent definition of DGF and analyzed the effect of DGF on graft function and survival independently. When DGF was diagnosed if the serum creatinine level increased, remained unchanged or decreased less than $10 \%$ per day immediately after surgery during three consecutive days for more than 1 week, 183 (23.2\%) patients experienced DGF and 551 (69.7\%) had primary graft function. If we defined DGF as the need of dialysis in the first week, 244 (33.9\%) of the patients would have been classified as having DGF. This means that $26 \%$ of patients that were dialyzed post-operatively required dialysis treatment for other reasons than DGF and that $10 \%$ of the patients experiencing DGF did not need dialysis treatment.

Studies on transplant outcomes have traditionally focused on patient- and graft survival as end-points without consideration of graft function. Although graft loss is the worst type of graft dysfunction, grafts with an impaired function require the most intense follow-up and therapeutic management and are economically most costly (11). For this reason graft function as a parameter in studies on outcome of kidney transplantation, should be considered.

One of the possible mechanisms of the decreased GFR in DGF seems related to tubular damage resulting from ischemia/reperfusion injury. Tubular epithelial cell degeneration, tubular cell exfoliation, interstitial edema and interstitial cellular infiltration are usually observed in biopsies in DGF (12). In the early phase, tubular obstruction by exfoliated tubular cells results in a low net. filtration pressure (13). Later, decreased sodium reabsorption results in afferent vasoconstriction and diminished glomerular filtration pressures through the tubulo-glomerular feedback mechanism (14). Another factor related to DGF is brain 
death (15), but all the patients studied received a cadaveric transplant.

In the present study we found that DGF was significantly associated with the use of kidneys from older donors, particularly donors of more than 50 years of age, with the use of female donor kidneys transplanted into male recipients, a cold ischemia time of more than 28 hours, historic panel reactive antibodies of more than $50 \%$ and a recipient's pretransplant MAP of less than $100 \mathrm{mmHg}$. Other authors have also reported an increased incidence of DGF in grafts from older donors $(1,16-18)$. In human adults, total metabolism and renal function in terms of glomerular filtration rate and renal blood flow, decrease with age. This is associated with a decrease in the number of glomeruli, a decrease in the mean glomerular volume (19) and interstitial fibrosis $(20,21)$. It is conceivable that such kidneys are more susceptible to additional insults such as brain death and the transplantation procedure.

The higher incidence of DGF in female donor to male recipient combinations could be explained by the absence of estrogens in the male environment. In-vitro studies have shown that the administration of estrogens leads to dilation of aortic rings (22) as has been described in-vivo in human coronary arteries (23). It is therefore conceivable that female kidneys when transplanted into a male environment experience more vasoconstriction and thus are more prone to DGF. An interesting observation is the finding that a low pretransplant blood pressure level in the recipient confers a significant risk to DGF (Table 2). A stable hemodynamic condition and possibly some degree of extra-cellular volume expansion are associated with good perfusion of the graft immediately after recirculation $(24,25)$. Moreover, invasive hemodynamic studies have shown that a high pulmonary artery (26) or central venous pressures (27) before, during and after the transplantation surgery correlates inversely with the incidence of DGF. As ischemia-reperfusion injury results in the loss of auto-regulation (28), the beneficial effect of hypervolemia may result in an increased glomerular perfusion flow and pressure. It is unknown, whether the reduced incidence of DGF in patients treated with peritoneal dialysis, as found by some authors $(29,30,30)$, is also based on an increased total extracellular fluid volume. A CIT of longer than 28 hours was also independently associated with an increased risk of DGF, as found by others (31-34). This is probably also the result of increased vasoconstriction (35) and renal damage as a result of ischemic injury. Peak panel reactive antibodies constitute another independent risk factors for DGF, as was noted by others (5). In studies in which DGF was defined as the need of dialysis within the first week after transplantation, DGF could theoretically have included acute rejection episodes. Although we corrected for acute rejection episodes, peak panel reactive antibodies remained independently correlated with DGF. It is thus conceivable that we missed some very early rejection episodes, as we did not biopsy every graft experiencing DGF, within 1 week. In contrast to another study (5), we found no effect of the initial immunosuppressive regimen on early graft function.

The transplanted nephron mass and subsequent graft damage determine renal function and many of the riskfactors for graft loss operate very likely through these factors $(36,37)$. We found that the number of acute rejection episodes, donor age, donor gender, DGF and decreased sharing of CREGs are correlated independently with graft function at 1 year. Using either 50 or $30 \mathrm{ml} / \mathrm{min}$ as the cut-off point, DGF remained a risk factor for poor graft function at 1 year. Although DGF is a strong risk factor for acute rejection episodes (OR 1.63 $95 \% \mathrm{Cl} 1.11-2.33$ ), the effect of DGF on graft function was independent from the number of rejection episodes. Long term follow-up studies of native kidneys that have experienced 
ATN suggested a decrease in renal function in most cases, albeit it was not associated with chronic failure $(38,39)$. However, experimental studies in rats have shown that ischemia added to ongoing injury results in more severe tissue damage (40). Some authors found an effect of DGF on graft survival only in combination with acute rejection episodes $(3,4,41)$. In our model, DGF had no influence on graft loss at 1 year or after the first year. Renal function at 1 year is probably a more important determinant for late graft loss, as suggested in the Collaborative Transplant Study (42). To further study the effect of DGF on late outcome, we stratified renal function after 1 year in 4 strata (fig. 3) and demonstrated that renal function at 1 year is a risk factor of late graft-loss. When the contribution of DGF on late graft loss was analyzed in these strata, there was no additional effect of DGF on outcome.

In this retrospective study we found that DGF, defined as the absence of a decline in serum creatinine of $10 \%$ or more in three consecutive days for more than 1 week after transplantation has an independent effect on graft function at one year as well as on the incidence of acute rejection episodes, but it does not seem to influence early or late graft loss. Graft survival after 1 year is mainly determined by the creatinine clearance at 1 year, which suggests that the influence of DGF on graft survival is through graft function at 1 year. We also found that the incidence of DGF is related to pretransplantation MAP, probably as a marker for the effective circulating volume. Furthermore, the use of kidneys from older donors or from female donors transplanted into male recipients increases the risk for DGF. It remains to be seen whether changing these risk factors improves the rate of DGF as well as long term function. 


\section{REFERENCES}

1. Ojo AO, Wolfe RA, Held PhJ, Port FK, Schmouder RL. Delayed graft function: risk factors and Implications for renal allograft survival. Transplantation 1997:63:968-974.

2. Shoskes DA, Cecka JM. Deleterious effects of delayed graft function in cadaveric renal transplant recipients independent of acute rejection. Transplantation 1998:66: 1697-1701.

3. Lehtonen SRK, Isoniemi HM, Salmela KT, Taskinen El, Von willebrand EO, Ahonen JP. Long-term graft outcome is not necessarily affected by delayed onset of graft function and early acute rejection. Transplantation 1997:64: 103-107.

4. Troppmann C, Gillingham KJ, Gruessner RWG et al. Delayed graft function in the absence of rejection has no long-term impact. Transplantation 1996:61:1331-1337.

5. Giralclasse $M$, Hourmant $M$, Cantarovich $D$ et al. Delayed graft function of more than six days strongly decreases long-term survival of transplanted kidneys. Kidney Int 1998:54:972-978.

6. Sund S, Reisaeter AV, Fauchald P, Bentdal O, Hall KS, Hovig T. Living donor kidney transplants: a biopsy study 1 year after transplantation, compared with baseline changes and correlation to kidney function at 1 and 3 years [In Process Citation]. Nephrol Dial Transplant 1999:14:2445-2454.

7. Woo YM, Jardine AG, Clark AF et al. Early graft function and patient survival following cadaveric renal transplantation. Kidney Int 1999:692-699.

8. Kooijmans-Coutinho MF, Hermans J, Schrama E et al. Interstitial rejection, vascular rejection, and diffuse thrombosis of renal allografts. Predisposing factors, histology, immunohistochemistry, and relation to outcome. Transplantation 1996:61:1338-1344.

9. Cockcroft DW, Gault MH. Prediction of creatinine clearance from serum creatinine. Nephron 1976:16: 31-41.

10. Truong L, Shappell S, Solez K. Adhesion molecules as markers of acute cellular rejection of renal allografts. [Review].Transplant Proc 1996:28:519-522.

11. Anonymous. The high cost of delayed graft function in cadaveric transplantation. Transplantation 1991:51:1115-1118.

12. Marcussen N, Lai R, Olsen S., Solez K. Morphometric and immunohistochemical investigation of renal biopsies from patients with transplant ATN, native ATN or acute graft rejection. Transplant Proc 1996:28: 470-476.

13. Donohoe JF, Venkatachalam MA, Bernard DB, Levinsky NG. Tubular leakage and obstruction after renal ischemia: structural-functional correlations. Kidney Int 1978:13:208-222.

14. Alejandro VS, Nelson WJ, Huie P et al. Postischemic injury, delayed function and Na+/K(+)-ATPase distribution in the transplanted kidney. Kidney Int 1995:48: 1308-1315.

15. van der Hoeven JA, Ploeg RJ, Postema F et al. Induction of organ dysfunction and up-regulation of inflammatory markers in the liver and kidneys of hypotensive brain dead rats: a model to study marginal organ donors. Transplantation 1999:68: 1884-1890.

16. Koning $\mathrm{OHJ}$, Ploeg RJ, van Bockel JH et al. Risk factors for delayed graft function in cadaveric kidney transplantation - A prospective study of renal function and graft survival after preservation with University of Wisconsin solution in multi-organ donors. Transplantation 1997:63: 1620-1628.

17. Pfaff WW, Howard RJ, Patton PR, Adams VR, Rosen CB, Reed AI. Delayed graft function after renal transplantation. Transplantation 1998:65:219-223.

18. Terasaki P, Gjertson DW, Cecka JM, ÿ, Cho YW. Significance of the donor age effect on kidney transplants. Clin Transplant 1997:11:366-372. 
19. Nyengaard JR, Bendtsen TF. Glomerular number and size in relation to age, kidney weight, and body surface in normal man. Anat Rec 1992:232: 194-201.

20. Rodriguez-Puyol D. The aging kidney. Kidney Int 1998:54:2247-2265.

21. Seron D, Diaz-Gallo C, Grino JM et al. Characterization of interstitial infiltrate in early renal allograft biopsies in patients with stable renal function. Transplant Proc 1991:23: Pt 2):1267-9.

22. Ma L, Yu Z, Xiao S, Thadani U, Robinson CP, Patterson E. Supersensitivity to serotonin- and histamineinduced arterial contraction following ovariectomy. Eur J Pharmacol 1998:23: 191-200.

23. Reis SE, Holubkov R, Zell KA et al. Estrogen acutely abolishes abnormal cold-induced coronary constriction in men. Chest 1998:114:1556-1561.

24. Dawidson IJ, Sandor ZF, Coorpender L et al. Intraoperative albumin administration affects the outcome of cadaver renal transplantation. Transplantation 1992:53:774-782.

25. Toth M, Reti V, Gondos T. Effect of recipients' peri-operative parameters on the outcome of kidney transplantation. Clin Transplant 1998:12:511-517.

26. Carlier M, Squifflet JP, Pirson Y, Gribomont B, Alexandre GPJ. Maximal hydration during anesthesia increases PAP and improves early function of human renal transplants. Transplantation 1982:34: 201204.

27. Thomsen HS, Lokkegaard H, Munck O. Influence of normal central venous pressure on onset of function in renal allografts. Scand J Urology Nephrology 1987:21:143-145.

28. Morita K, Seki T, Nonomura K, Koyanagi T, Yoshioka M, Saito H. Changes in renal blood flow in response to sympathomimetics in the rat transplanted and denervated kidney. Int J Urol 1999:6:24-32.

29. Vanholder $R$, Heering $P$, Vanloo $A$ et al. Reduced incidence of acute renal graft failure in patients treated with peritoneal dialysis compared with hemodialysis. Amer J Kidney Dis 1999:33: 934-940.

30. Velasquez MT, Lew SQ, von Albertini B, Mishkin GJ, Bosch JP. Control of hypertension is better during hemodialysis than during continuous ambulatory peritoneal dialysis in ESRD patients. Clin Nephrol 1997:48: 341-345.

31. Jacobs U, Niese D, Klein B, Paar D, Miersch WD, Klehr HU. Cold ischemia, histocompatibility, donor and recipeints age: impact on early lymphocyte subsets and transplant outcome. Transplant Proc 1996:28: 3251-3252.

32. Kahan B., Mickey R., Flechner S.M. et al. Multivariate Analysis of risk Factors Impacting on Immediate and Eventual Cadaver Allograft Survival in Cyclosporine-Treated Recipients. Transplantation 1987:43: 65-70.

33. Lechevallier E, Dussol B, Luccioni A et al. Posttransplantation acute tubular necrosis: Risk factors and implications for graft survival. Amer J Kidney Dis 1998:32: 984-991.

34. Peters ThG., Shaver TR, Ames JE, Santiago-Delpin EA, Jones KW, Blanton JW. Cold ischemia and outcome in 17.937 cadaveric kidney transplants. Transpl Immunol 1995:59: 191-196.

35. Conger J, Weil JV. Abnormal vascular function following ischemia-reperfusion injury. J Investig Med 1995:43:431-442.

36. Azuma H, Nadeau K, Takada M, Mackenzie HS, Tilney NL. Cellular and molecular predictors of chronic renal dysfunction after initial ischemia/reperfusion injury of a single kidney. Transplantation 1997:64: 190-197.

37. Hostetter TH, Olson JL, Rennke HG, Venkatachalam MA, Brenner BM. Hyperfiltration in remnant nephrons: a potentially adverse response to renal ablation. Am J Physiol 1981:241: F85-F93.

38. Briggs JD, Kennedy AC, Young LN, Luke RG, Gray M. Renal function after acute tubular necrosis. Br Med J 1967:3:513-516.

39. Lewers DT, Mathew TH, Maher JF, Schreiner GE. Long-term follow-up of renal function and histology 
after acute tubular necrosis. Ann Intern Med 1970:73:523-529.

40. Yilmaz S, Paavonen T, Hayry P. Chronic rejection of rat renal allografts. II. The impact of prolonged ischemia time on transplant histology. Transplantation 1992:53: 823-827.

41. Troppmann C, Gillingham KJ, Benedetti E et al. Delayed graft function, acute rejection and outcome after cadaveral renal transplantation. Transplantation 1995:59:962-968.

42. Opelz G. Immunosuppression with FK 506 does not improve kidney graft survival. Collaborative Transplant Study. Transplant Proc 1999:31:1147-1148. 


\section{DELAYED GRAFT FUNCTION IS}

CHARACTERIZED BY REDUCED

FUNCTIONAL MASS MEASURED BY ${ }^{99}$ TC-MAG3 RENOGRAPHY

Henk Boom,Tarek A.F. El-Maghraby, Jan A.J.Camps, Koos A.K. Blokland, Aeilko H.Zwinderman, Leendert C. Paul, Ernest K.J. Pauwels, Johan W. de Fijter.

Transplantation 2002;74:203-208 
Background The mechanism that underlies delayed graft function (DGF) is still poorly defined. Previous studies using tubular function tests have shown that post-ischemic injury to the renal transplants results in profound impairment of para-immunohippurate extraction by the tubules.

Methods Using ${ }^{99 m}$ Technetium-mercaptoacetyltriglycine $\left.{ }^{99 m} T c-M A G 3\right)$ renography and tubular function slope (TFS) we studied the tubular uptake of ${ }^{99 m} \mathrm{Tc}-\mathrm{MAG} 3$ in a prospective study of renal transplant recipients with immediate graft function (IGF) and those with DGF.

Results Thirty-seven consecutive recipients of a cadaveric graft and 5 kidneys from living donors were evaluated within 48 hours after transplantation and in week 2, month 3 and 6 and 3 years after transplantation. In addition to the protocol scans, recipients with DGF were examined every other day until function was resumed. Repeated measurement twoway ANOVA and a change point analysis were performed to determine the difference in the follow-up of TFS values between the two groups. Fourteen patients were classified as having DGF and 28 IGF. In the DGF group, the initial TFS value was significantly lower than in the IGF group $[0.54(+0.01)$ and $1.75(+0.16)$ respectively; $P=0.002]$, a difference that persisted for up to 3 years. Change point analysis revealed that the post ischemic tubular excretion improved with time in both groups in the first 3 to 4 weeks, but both groups remained different up to 3 years after transplantation. Multi-variate analysis revealed that only the cold ischemic time (CIT) was an independent risk factor for a low TFS value. After the initial recovery from post-ischemic injury, the TFS may be used as a marker for functional renal mass.

Conclusion We propose that the tubular defect in DGF defined by ${ }^{99 m}$ Technetium-mercaptoacetyltriglycine $\left.{ }^{99 m} T c-M A G 3\right)$ renography is irreversible and may be a marker of initial graft function. 


\section{INTRODUCTION}

Despite increased donor awareness and actions, the actual number of organ donors has not increased to any meaningful extend. This disparity has led to an increased interest in the use marginal donors, including donors at the extremes of age, non-heart beating donors and donors with a history of hypertension or diabetes $(1,2)$.

DGF is a common complication after renal transplantation, with a reported incidence of up to $50 \%$, especially in recipients of kidneys from marginal donors (3-6). The underlying cause is usually ischemic damage, which may be further complicated by an increased likelihood of acute rejection episodes $(3,7)$ or drug-related nephrotoxicity (8). There is still debate on the impact of DGF on late graft outcome. Some authors find an effect of DGF on renal allograft survival $(9,10)$, but others only find an effect of DGF on graft survival in patients who experience acute rejection episodes (11).

To answer the question whether the occurrence of DGF is merely an expression of an insufficient amount of functional renal mass at the time of transplantation or an independent risk factor for subsequent graft loss, there is need for a marker of renal mass that is easy assessable and can be repeated frequently. Several studies have been undertaken to define the nature of graft dysfunction in DGF. Corrigan et al. found in human renal transplants with post-ischemic injury, a severely impaired para-aminohippurate (PAH) extraction, by the renal tubules that persisted for up to 7 days (12). The renal handling of ${ }^{99 m}$ Technetium-mercapto-acetyltriglycine ( ${ }^{99 m} \mathrm{Tc}-\mathrm{MAG} 3$ ) is similar to that of PAH (13). We prospectively evaluated serial ${ }^{99 m}$ Tc-MAG3 renographies in patients with immediate or delayed graft function, using a standardized method to estimate the active tubular extraction for up to 3 years.

\section{MATERIALS AND METHODS}

Between August 1997 and January 1998, 37 consecutive recipients of a first or second cadaveric renal transplant and 5 recipients of a living related donor transplant gave informed consent and were entered into the study. DGF was defined based on previously described criteria (3) and never functioning grafts were excluded from the study. In short, cases were categorized as DGF when the serum creatinine level increased, remained unchanged, or decreased by less than $10 \%$ per day immediately following surgery during three consecutive days within the first week. All other situations were designated as immediate graft function (IGF). An acute rejection episode as a cause of graft dysfunction was defined as a decline in renal function by more than $10 \%$ and was confirmed by a percutaneous renal biopsy unless this could not be obtained. Cyclosporine (CsA) nephrotoxicity was diagnosed based on the criteria described in the Banff 1997 classification of renal allograft pathology (14) or when there was a normalization of renal function after reduction of the CsA dose. Renal function, expressed as the creatinine clearance was estimated using the formula developed by Nankivell, which showed a good correlation with ${ }^{99 m} \mathrm{Tc}$ diethylenetriaminepentaacetate ${ }^{99 m}$ Tc DTPA) clearance (15): 
GFR $[\mathrm{ml} / \mathrm{min}]=6.7 /$ creat. $[\mathrm{mmol} / \mathrm{l}]+\mathrm{BW}[\mathrm{kg}] / 4$ - urea $[\mathrm{mmol} / \mathrm{l}] / 2-100 /(\text { height }[\mathrm{m}])^{2}+$ (35 [ male] or 25 [ female]).

All patients had an initial ${ }^{99 m}$ Tc-MAG3 study on the first post-transplant day. Protocol scans were performed at 2 weeks, 3 and 6 months and at three years after transplantation. In addition, patients experiencing DGF were followed with ${ }^{99 m}$ Tc-MAG3 studies every other day until the serum creatinine concentration decreased by more than $10 \%$ per day on three consecutive days.

${ }^{99 m}$ Tc-MAG3 renogram

${ }^{99 m}$ Tc-MAG3 studies were done by positioning a large field of a view gamma camera (Toshiba GCA 501S) anteriorly over the patient in the supine position. The field of view included the transplanted kidney, the lower abdominal aorta and iliac arteries as well as

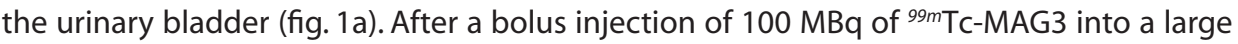
caliber vein (usually the medial antecubital vein), frames were recorded initially at $1 \mathrm{sec}$ intervals for 120 frames, followed by 90 frames at $20 \mathrm{sec}$ intervals to complete the 32 minute study as shown in figure $1 \mathrm{~b}$ and $2 \mathrm{a}$. To calculate the dose activity administered, the syringe was counted before and after injection and when there was extravasation, the injection site was counted as well. A dedicated nuclear medicine computer (MAPS 10000 Web Link Medical, UK) was programmed for processing the data and calculating the Tubular Function Slope (TFS).

\section{Tubular Function Slope}

To study the radiopharmaceutical uptake by the renal tubular cells the tubular function slope (TFS) was designed. Two regions of interest were drawn semi-automatically; one around the graft ( $\mathrm{ROI}$ - 1) and one representing the background (ROI - 2) (fig. 1a). The background region of interest was crescentic in shape and it was placed infero-lateral to the kidney, avoiding any vascular structures (fig. 1a). A background subtracted graft curve was generated during the first two minutes of the study. (fig. 2 a): it consists of a rapidly ascending initial phase until the first pass peak, which results from the initial perfusion, followed by a second phase which represents the tubular extraction phase (fig. 2 b). The graft curve was normalized to the injected dose and a measure of the slope was taken using a linear fit (least squared error estimate) of the curve between 50 seconds and 110 seconds (figure 2 b). This slope was defined as the TFS. As TFS is determined in the second phase of the time activity curves it is proposed to be independent of renal perfusion.

\section{Reproducibility and inter-observer reliability}

To assess the intra-observer variability the TFS was calculated twice in two different sessions by one observer in a random set of 33 scintigrams of 10 patients. The average difference between the TFS values between the first and second processing was 0.062 (SD 0.016 ) with a correlation of 0.992 . The intra-class correlation was 0.991 . The inter-observer variability was assessed by processing 59 scans twice by two different operators. The aver- 
Figure 1

a: ${ }^{99 m}$ Tc-MAG3 renogram. The region of interest 1 (ROI -1) describes the whole kidney activity. The region of interest 2 (ROI - 2) describes the avascular reference region.

b: Backgound subtracted and dose adjusted ${ }^{99 m}$ Tc-MAG3 extraction and secretion curve of a kidney experiencing immediate graft function

\section{Anterior}

A

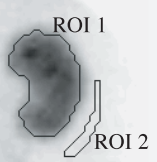

right

left

B

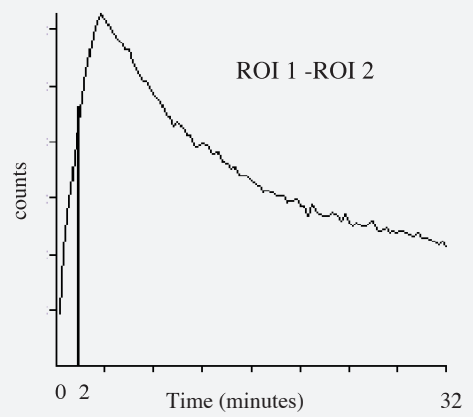

A

Figure 2

a: Backgound subtracted and dose adjusted ${ }^{99 m}$ TC-MAG3 extraction and secretion curve of kidney experiencing delayed graft function. (Note: time axis is until 32 minutes).

b: First 2 minutes of the ${ }^{99 m}$ Tc-MAG3 extraction and secretion curve in which Phase 1 (the rapidly ascending phase) represents the renal perfusion phase. Phase 2 represents the tubular extraction phase. Using a linear fit (least squared error estimate) the slope of this curve is determined and defined as tubular function slope (TFS).

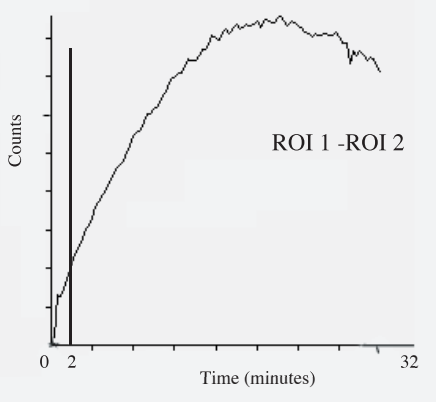

B

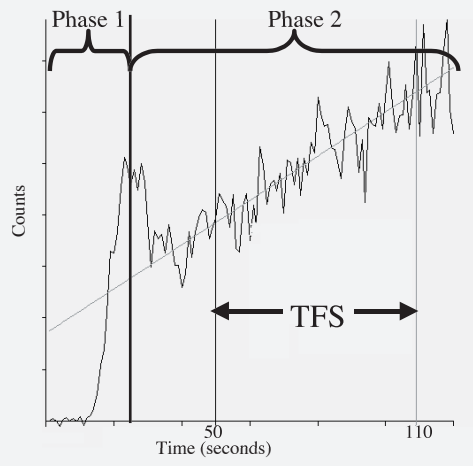


age difference in TFS values between the two was 0.011 (SD 0.019) with a correlation of 0.997. The inter-class correlation was estimated to be 0.997 . These excellent correlations were not unexpected as the calculations of the TFS, including drawing the regions of interest and the defined periods in the second phase of the early dynamic curve, are almost entirely automatic.

\section{Immunosuppressive Regimen}

The standard immunosuppressive regimen consisted of Prednisone, Cyclosporine (Neoral) and Mycophenolate Mofetil (MMF). Cyclosporine was administered intravenously in a dose of $3 \mathrm{mg} / \mathrm{kg} /$ day for the first 48 hours, starting at the onset of surgery. The initial oral dose of Neoral was $10 \mathrm{mg} / \mathrm{kg} /$ day from day 2 onward. Subsequent doses were adjusted according to cyclosporine 12-hour trough level monitoring. Mean cyclosporine dose and trough levels were not significantly different between the 2 groups. In the first three months, the target 12-hour trough level was $300 \mu \mathrm{g} / \mathrm{l}$ (range $250 \mu \mathrm{g} / \mathrm{l}-350 \mu \mathrm{g} / \mathrm{l}$ ) and beyond 3 months it was $150 \mu \mathrm{g} / \mathrm{l}$ (range $100 \mu \mathrm{g} / \mathrm{l}-200 \mu \mathrm{g} / \mathrm{l}$ ). All patients started on $20 \mathrm{mg} /$ day of Prednisolone followed by a dose reduction of $2.5 \mathrm{mg}$ every fortnight until the maintenance dose of $10 \mathrm{mg}$ was reached. MMF, 1 gram b.i.d. was given from day 1 onward. Acute rejection episodes were treated with $1 \mathrm{gram}$ of methylprednisolone intravenously for 3 consecutive days. Severe or steroid resistant acute rejection episodes or any second rejection episode were treated with rabbit anti-thymocyte globulin for 10 days, as previously described (16).

\section{Statistical analysis}

Risk factors for a low TFS were analyzed, using linear regression analysis. Differences in TFS and creatinine clearance were examined using the two way ANOVA for repeated measurements. Furthermore, the change of the $\ln$ (TFS)-parameter during follow-up was modeled using a change-point model with two linear regression lines, one involving a rapid increase after transplantation, and a second line, following a change-point, involving a slow change of the $\ln$ (TFS) parameter. The parameters of the regression lines, and the timing of the change-point was assumed to vary between patients. These parameters were handled as random, drawn from the multivariate normal distribution. The mean and (co)variance of this normal distribution were estimated separately for patients with immediate and delayed graft function. The change of the In(TFS) of the "averaged" patient according to the model thus defined, was calculated and illustrated graphically. A p-value of 0.05 or less was considered significant. Statistical analysis was done using the SPSS 9.0 software package (Version 9.0; SPSS, Inc., Chicago, IL).

\section{RESULTS}

A total of 42 patients were prospectively followed with ${ }^{99 m}$ Tc-MAG3 renography; 28 experienced IGF and 14 had DGF. The characteristics of the study population, grouped according to the presence or absence of DGF are summarized in Table 1. In this cohort of patients none of the factors summarized in Table 1 were statistically different between 
the two groups. Although donor age and the number of female donors were slightly higher in the DGF group, this did not reach significance. The type of rejection episodes was not different in the two groups. Three patients in the IGF group experienced recurrence of their renal disease (IgA nephropathy, membrano proliferative glomerulonephritis and focal segmental glomerulosclerosis) and one lost his graft after 432 days. Two patients in the DGF group lost their graft because of recurrence of their renal disease (micro angiopathic nephropathy and membrano proliferative glomerulonephritis) after 43 and 373 days respectively. A total of two hundred and seventy three ${ }^{99 m} \mathrm{TC}-M A G 3$ renographies were performed: 160 in the IGF group and 113 in the DGF group.

Table 1. Patient characteristics grouped according to the incidence of DGF

\begin{tabular}{|c|c|c|c|}
\hline Characteristic & $\begin{array}{l}\text { IGF } \\
(\mathrm{N}=28)\end{array}$ & $\begin{array}{l}\text { DGF } \\
(N=14)\end{array}$ & p-value \\
\hline \multicolumn{4}{|l|}{ Pretransplantation factors } \\
\hline HLA-AB mismatch & 1.25 (1.07) & $0.92(0.86)$ & 0.37 \\
\hline HLA-DR mismatch & $0.46(0.51)$ & $0.38(0.51)$ & 0.67 \\
\hline Panel reactive antibodies (historic) & $30(33)$ & $19(27)$ & 0.30 \\
\hline Donor age, years & $46(17)$ & $48(11)$ & 0.81 \\
\hline Recipient age, years & $45(13)$ & $44(11)$ & 0.80 \\
\hline \multicolumn{4}{|l|}{ Gender } \\
\hline Recipient male (\%) & 64.3 & 78.6 & 0.34 \\
\hline Donor female (\%) & 50 & 35.7 & 0.38 \\
\hline Mismatch: female to male (\%) & 32.1 & 35.7 & 0.91 \\
\hline Cold ischemia time, (hours) & $23(8)$ & $25(5)$ & 0.46 \\
\hline Warm ischemia time (minutes) & $35(12)$ & $36(11)$ & 0.80 \\
\hline Pretransplantation MAP, $(\mathrm{mmHg})$ & $105(16)$ & $104(12)$ & 0.70 \\
\hline Cadaveric transplantation (\%) & 82 & 100 & 0.15 \\
\hline \multicolumn{4}{|l|}{ Cause of donor death } \\
\hline Cardiovascular (\%) & 61 & 64 & 1.00 \\
\hline Retransplants (\%) & 21 & 14 & 0.39 \\
\hline \multicolumn{4}{|l|}{ Posttransplantation factors } \\
\hline \multicolumn{4}{|l|}{ Immunosuppression } \\
\hline Pred / neoral/ MMF (\%) & 100 & 100 & 1.00 \\
\hline Biopsy proven rejection episodes (\%) & 53 & 36 & 0.34 \\
\hline
\end{tabular}

Data are expressed as \pm SD, unless otherwise stated; MAP: mean arterial pressure; MMF:Cellcept; Pred.: prednisone 
Follow-up of TFS

The TFS values after predefined time intervals for both the IGF and the DGF group are shown in figure 3a. In the DGF group the average TFS values (mean \pm SEM) in the initial scans was $0.54 \pm 0.01$ and there was an increase to $1.8 \pm 0.19$ in the week 2 studies. In the IGF group the average TFS values (mean \pm SEM) at the first examination and the week two studies were $1.75 \pm 0.16$ and $2.52 \pm 0.22$, respectively. At the month 3 and 6 and year 3 time points, the initial difference in TFS values between the two groups persisted; for the DGF group the TFS values were $1.83 \pm 0.17,1.84 \pm 0.19$ and $1.38 \pm 0.24$ respectively and for the IGF group they were $2.91 \pm 0.22,3.17 \pm 0.23$ and $2.76 \pm 0.31$. The differences between the two groups were highly significant $(P<0.0001)$. After correction for the initial TFS value, by subtracting the initial TFS values from the follow-up TFS values of each group, these differences were no longer present $(p=0.85)$ and the curves were superimposable. These data show that in both groups, despite of comparable recovery of tubular function as measured by TFS, there is an initial difference in the TFS values that persists for up to 3 years.

\section{Change point model}

To further evaluate whether there was a difference in time to reach the maximum TFS values between the two groups, all 273 available scans were studied in a change-point analysis model. The results of this analysis are plotted in figure 4. The slopes of the sharp increasing regression lines prior to the change-point were not different for recipients with DGF and patients with IGF (0.06 In(TFS-units)/day and $0.06 \ln$ (TFS-units)/day respectively; $p$ $=0.87$ ), but the absolute level achieved was different between the two groups ( $P=0.005)$. Interestingly the timing of the change-point was also the same in DGF-and IGF-patients $(29 / 21$ days, $p=0.85)$ as was the slow $\ln ($ TFS)-increase thereafter $[-0.0003 \ln$ (TFS) units and $-0.00002 \ln$ (TFS) units $(p=0.24)$ ]. This means that TFS remains at a constant level from about 3 to 4 weeks onwards both in the presence or the absence of DGF.

\section{Factors influencing the TFS value and renal function}

To evaluate the risk factors that correlated with an initial low TFS value and the creatinine clearance and TFS values at the predefined time-points, several factors concerning the donor and the transplantation procedure were retrospectively evaluated. In the multivariate analysis, the only significant risk factor for a low post-transplant TFS value was the cold ischemia time (correlation coefficient (CC):- 0.27 per hour; $-0.54-0.00$ ). In the multivariate analysis, only the TFS value at 6 months was correlated with the TFS value found after 3 years (CC: $0.75 ; 0.58-1.16$ ) and the estimated creatinine clearance at 1 year was the only variable that was correlated with the graft function at 3 years (CC: $0.875 ; 0.59-0.89$ ). The creatinine clearances at 3 and 6 months and 1 and 3 years are plotted in figure $3 \mathrm{~b}$ and were $56 \mathrm{ml} / \mathrm{min}, 60 \mathrm{ml} / \mathrm{min}, 58 \mathrm{ml} / \mathrm{min}$ and $58 \mathrm{ml} / \mathrm{min}$ for the DGF group and $64 \mathrm{ml} / \mathrm{min}, 71 \mathrm{ml} /$ $\mathrm{min}, 67 \mathrm{ml} / \mathrm{min}$ and $63 \mathrm{ml} / \mathrm{min}$ for the IGF group. The estimated clearances tended to be lower in the DGF group, but this did not reach a statistical significance $(p=0.20)$, because of the limited sample size. 
Figure 3

a: TFS values in the two groups, which are different $(p=0.005)$. After correction for the initial TFS value, by subtracting the inital TFS values from the TFS values in the follow-up of each group, these differences were no longer present $(p=0.85)$ and the curves were superimposable, indicating that the differences between the two groups were determined by the differences already present in the early post transplant ${ }^{99 m}$ Tc-MAG3 renographies

( IGF: dashed rule ;DGF: solid rule )

b: Creatinine clearances at the different time points. The values tended to be lower in patients that experienced DGF but did not reach statistical difference $(p=0.2)$. (IGF: dashed rule ;DGF: solid rule).
A

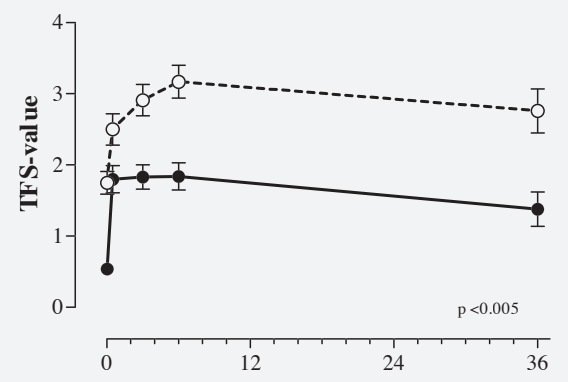

B

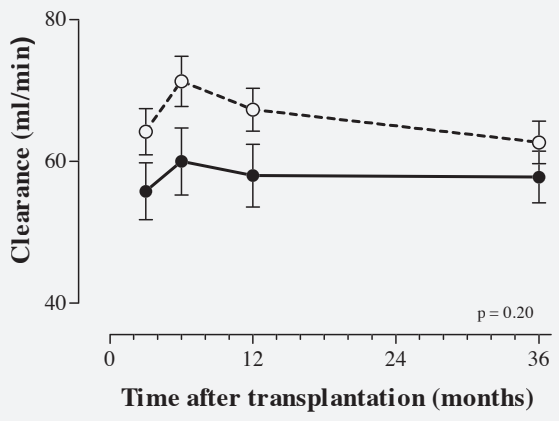

Figure 4

The change point model, using all 273 available renographies. The improving of the tubular extraction impairment occurred within the first post-transplant month and to the same extend in both groups.

(IGF: dashed rule ;DGF: solid rule)

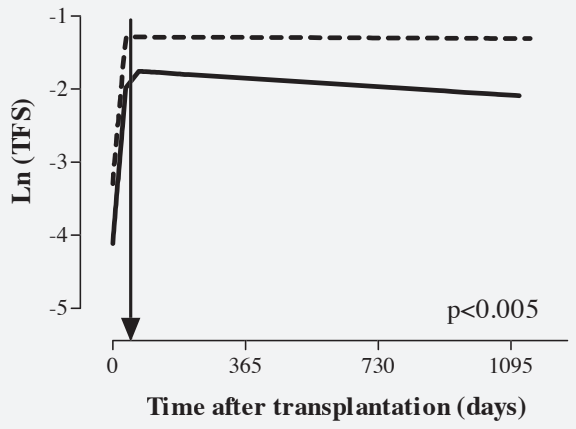


Use of TFS in detection of acute rejection episodes or cyclosporine toxicity

In the total study population 24 episodes were identified with a decline of TFS of more than $20 \%$ in 2 consecutive ${ }^{99 m}$ Tc-MAG3 renographies performed within the first three weeks. In $17(71 \%)$ cases an acute rejection episode or cyclosporine toxicity was identified and in 7 (29\%) cases no obvious explanation could be found. In the DGF group there were 14 cases with a decline of TFS of more than $20 \%$ in 2 consecutive scans. In 10 (71\%) cases an acute rejection episode or acute cyclosporine toxicity was identified, in 4 (29\%) cases no obvious explanation was found. Figure 5 shows two representative examples of the relation between the decline of TFS and an acute rejection episode (fig $5 \mathrm{a}$ ) and he TFS and cyclosporine toxicity (5b) in patients who experienced DGF. Both curves show complete reversibility of the TFS value after treatment of the acute rejection episode or lowering of the CsA dose respectively.

Figure 5

a: A representative case of an acute rejection episode ( Grade II a) in a patient experiencing DGF. TFS values are reversible after treatment.

b: $A$ representative case of an episode of cyclosporine toxicity (CyA 12 hours trough level: $600 \mu \mathrm{mol} / \mathrm{l}$ ) in a patient with DGF. TFS values are reversible after correction of the neoral dose.

\section{Cyclosporin toxicity}

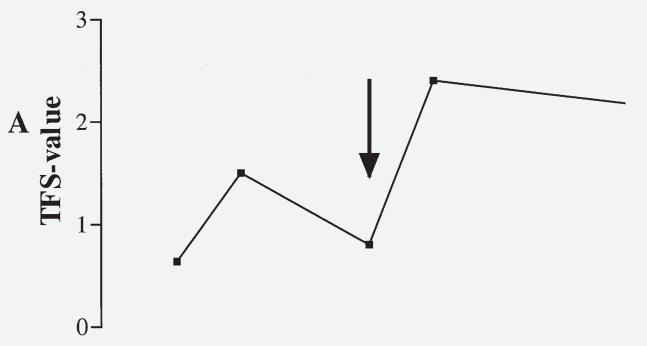

0

Acute rejection

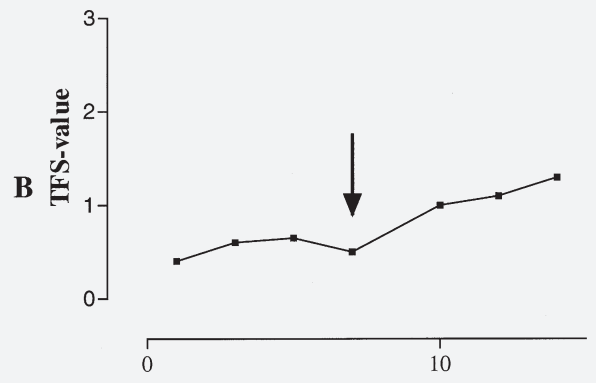

Days after transplantation 


\section{DISCUSSION}

In the present study we demonstrated that transplantation of a renal allograft is associated with a low level of active tubular extraction of the radiopharmaceutical ${ }^{99 m}$ Tc-MAG3 as assessed by renography. The tubular extraction is expressed as the tubular function slope (TFS). The TFS values were particularly decreased in the immediate post-operative days in recipients who experienced delayed graft function. Independent of whether the graft showed immediate good function or delayed function, this tubular extraction impairment of ${ }^{99 \mathrm{~m}} \mathrm{Tc}-\mathrm{MAG} 3$ showed improvement within the first post-transplantation weeks in both groups, but remained always lower in the DGF group.

After the first post-transplant month, the difference in the absolute TFS values between recipients with immediate function and those with delayed function persisted in subsequent ${ }^{99 m}$ Tc-MAG3 renographies for up to three years. The results where not different when the data of the living related transplants or the data of the grafts that showed recurrence of their renal disease were left out of the analysis.

Previously, a firm correlation between histological tubulo-interstitial changes and renal outcome has been established (17). Since the TFS is a marker of the proximal tubular function and the tubular compartment represents approximately 80 to $85 \%$ of the renal mass, we propose that it may serve as a marker of renal mass after the initial recovery from postischemic injury.

It has been difficult to quantitate the amount of renal mass transplanted. The degree of glomerulosclerosis and glomerular size have been proposed as a measure of functional renal mass but these parameters have a poor correlation with outcome $(18,19)$. However as stated before, there is a good correlation between tubulo-interstitial changes and outcome (17).

Radionuclide renography, using ${ }^{99 m}$ Tc-MAG3 is a non-invasive method that can be repeated frequently. To improve its diagnostic accuracy, various quantitative parameters have been proposed $(20,21)$. In ${ }^{99 m} \mathrm{Tc}-M A G 3$ renography the second part of the early phase of the curve represents the active extraction of the radiopharmaceutical by epithelial cells of the proximal renal tubules (22-26). No objective correlations with graft dysfunction have been established yet $(23,26,27)$. Therefore, we defined the tubular function slope (TFS) in ${ }^{99 m}$ Tc-MAG3 renography, as a putative objective marker of tubular extraction capacity. The hypothesis that the TFS value of the renogram measures active tubular excretion is supported by studies using para-aminohippurate (PAH). Since the renal handling of ${ }^{99 \mathrm{~m} T \mathrm{TC}-M A G 3}$ shares the same characteristics with the handling of PAH (13), it is likely that both substances are handled similarly. In a study of transplanted human kidneys with ischemic injury, no correlation between the PAH clearance and renal plasma flow, measured by phase contrast-cine-magnetic resonance imaging (MRI), was found (12). The authors concluded that the severely depressed clearance was explained by the reduced tubular extraction of the PAH. Because the tubular extraction of ${ }^{99 m} \mathrm{Tc}-\mathrm{MAG} 3$ is an energy dependent transport mechanism and occurs along the entire length of the proximal tubules, it will be affected by ischemic injury or inflammation. These conditions are characterized by the loss of tubular cell polarity, which impairs the transport of ${ }^{99 m} \mathrm{Tc}-\mathrm{MAG} 3$ by the dislocation of the $\mathrm{Na}+-\mathrm{K}+$ -ATP-ase from the basolateral cell membrane into the cytoplasm $(28,29)$. 
A decrease in TFS value of more than $20 \%$ in consecutive ${ }^{99 m}$ Tc-MAG3 renographies in the early post-transplant period can be helpful in detecting additional complicating factors, such as an acute rejection episode or cyclosporine toxicity. As these events also influence long term graft outcome $(3,7,8)$, we looked at the reversibility of the TFS values after treatment of an acute rejection episode or after adjusting the neoral dose. We found that TFS values were completely reversible in both conditions and we therefore assume that these events have a no major influence on the TFS values, during follow up.

We found that cold ischemic time (CIT) is an independent risk factor for a low initial TFS value in both groups. CIT has been reported as an independent risk factor for the occurrence of DGF(3,30-32). We hypothesize that in the presence of a long CIT, the quality and the amount of the functional renal mass transplanted, explains why some grafts experience DGF and others do not. It remains to be seen whether other risk factors for the occurrence of DGF, like donor age, pretransplant mean arterial pressure (MAP) and gender mismatch (3) are correlated with a low initial TFS-value when the numbers are increased.

We conclude that the TFS as defined in ${ }^{99 m}$ Tc-MAG3 renography may serve as a marker for the functional renal mass. Most renal transplants will experience a recovery phase from post-ischemic injury, but grafts from marginal donors are more likely to experience delayed graft function. After the initial recovery, grafts experiencing DGF have lower TFS values, than grafts without DGF, which remained evident up to 3 years of follow-up. Therefore, the TFS after for example 1 month provides information about renal mass that is not obtained by measures of glomerular filtration rate and may serve as a measure for initial renal mass post-transplantation. Furthermore a decrease of TFS in subsequent ${ }^{99 m}$ Tc-MAG3 renographies helps in the management of grafts experiencing DGF by planning additional diagnostic procedures like doing a renal biopsy or adapting calcineurine dose. Previous findings showed that DGF was associated with an increased risk of acute rejection episodes and a suboptimal function at 1 year but DGF alone was not independently associated with graft loss (3).The current study confirms that delayed graft function is associated with an initially decreased renal tubular function that appears irreversible. 


\section{REFERENCES}

1. Cecka JM and Terasaki PI. The UNOS scientific renal transplant registry, in Clinical Transplants 1996, edited by Terasaki PI, Cecka JM, Los Angeles, UCLA Tissue Typing Laboratory. 1997; 1-15.

2. Cohen B, Persijn GG, and De Meester J. Annual Report 1998 Leiden, Eurotransplant Foundation, 1999.

3. Boom H, Mallat MJ, De Fijter JW, Zwinderman AH, Paul LC. Delayed graft function influences renal function, but not survival. Kidney Int 2000:58: 859-866.

4. Koning $\mathrm{OHJ}$, Ploeg RJ, van Bockel JH et al. Risk factors for delayed graft function in cadaveric kidney transplantation - A prospective study of renal function and graft survival after preservation with University of Wisconsin solution in multi-organ donors. Transplantation 1997:63: 1620-1628.

5. Moreso F, Seron D, Gilvernet S et al. Donor age and delayed graft function as predictors of renal allograft survival in rejection-free patients. Nephrol Dialysis Transplant 1999:14: 930-935.

6. Ojo AO, Wolfe RA, Held PhJ, Port FK, Schmouder RL. Delayed graft function: risk factors and implications for renal allograft survival. Transplantation 1997:63: 968-974.

7. Halloran PF, Homik J, Goes N et al. The "injury response": a concept linking nonspecific injury, acute rejection, and long-term transplant outcomes. Transplant Proc 1997:29: 79-81.

8. Kahn D, Botha JF, Pascoe MD, Pontin AR, Halkett J, Tandon V. Withdrawal of cyclosporine in renal transplant recipients with acute tubular necrosis improves renal function. Transpl Int 2000:13 Suppl 1:S82S83.

9. Giralclasse $M$, Hourmant $M$, Cantarovich $D$ et al. Delayed graft function of more than six days strongly decreases long-term survival of transplanted kidneys. Kidney Int 1998:54:972-978.

10. Shoskes DA, Cecka JM. Deleterious effects of delayed graft function in cadaveric renal transplant recipients independent of acute rejection. Transplantation 1998:66: 1697-1701.

11. Troppmann C, Gillingham KJ, Benedetti E et al. Delayed graft function, acute rejection and outcome after cadaveral renal transplantation. Transplantation 1995:59: 962-968.

12. Corrigan $\mathrm{G}$, Ramaswamy D, Kwon $\mathrm{O}$ et al.PAH extraction and estimation of plasma flow in human postischemic acute renal failure. Am J Physiol 1999:277: F312-F318.

13. Muller-Suur C, Muller-Suur R. Handling of ${ }^{99 m}$ Tc-MAG3 in the kidney. Contrib Nephrol 1990:79:17-20.

14. Racusen LC, Solez K, Colvin RB et al. The Banff 97 working classification of renal allograft pathology. Kidney Int 1999:55: 713-723.

15. Nankivell BJ, Gruenewald SM, Allen RD, Chapman JR. Predicting glomerular filtration rate after kidney transplantation. Transplantation 1995:59: 1683-1689.

16. De Fijter, JW, Mallat, MJK, Doxiadis, IIN, Ringers, J, Rosendaal, FR, Claas, FHJ, and Paul, LC. Increased immunogenicity and cause of graft loss of old donor kidneys. J Am Soc Nephrol 12 2001; 1538-1546.

17. Cameron JS. Tubular and interstitial factors in the progression of glomerulonephritis. Pediatr Nephrol 1992:6: 292-303.

18. Gaber LW, Moore LW, Alloway RR, Amiri MH, Vera SR, Gaber AO. Glomerulosclerosis as a determinant of posttransplant function of older donor renal allografts. Transplantation 1995:60: 334-339.

19. Abdi R, Slakey D, Kittur D, Burdick J, Racusen L. Baseline glomerular size as a predictor of function in human renal transplantation. Transplantation 1998:66:329-333.

20. El maghraby, De Fijter JW, Van Eck Smit BLF, Zwinderman AH, El haddad SI, Pauwels EKJ. Renographic indices for evaluation of changes in graft function. Eur J Nucl Med 1998:25: 1575-1586.

21. Heaf JG, Iversen J. Uses and limitations of renal scintigraphy in renal transplantation monitoring. Eur J Nucl. Med. 2000:27:871-879. 
22. Bubeck B, Brandau W, Weber E, Pomer S, Georgi P, zum Winkel K. Renal function studies using ${ }^{99 m}$ TcMAG3: pharmacokinetics and slope clearance determination. Contrib Nephrol 1990:79:72-73.

23. Eshima D, Taylor JrA. Technetium- ${ }^{99 m}\left({ }^{99 m} \mathrm{Tc}\right)$ mercaptoacetyltriglycine: update on the new ${ }^{99 m} \mathrm{Tc}$ renal tubular function agent. Semin Nucl Med 1992:22:61-73.

24. Muller-Suur R, Bois-Svensson I, Mesko L. A comparative study of renal scintigraphy and clearance with technetium- ${ }^{99 m}$-MAG3 and iodine-123-hippurate in patients with renal disorders. J Nucl Med 1990:31: 1811-1817.

25. Taylor Jr. A, Eshima D, Christian PE, Wooten WW, Hansen L, McElvany K. Technetium- ${ }^{99 m}$ MAG3 kit formulation: preliminary results in normal volunteers and patients with renal failure. J Nucl Med 1988:29: 616-622.

26. Lin E, Alavi A.Significance of early tubular extraction in the first minute of Tc- ${ }^{99 m}$ MAG3 renal transplant scintigraphy. Clin Nucl Med 1998:23:217-222.

27. O'Malley JP, Ziessman HA, Chantarapitak N. Tc- ${ }^{99 m}$ MAG3 as an alternative to Tc- ${ }^{99 m}$ DTPA and I-131 hippuran for renal transplant evaluation. Clin Nucl Med 1993:18: 22-29.

28. Alejandro VS, Nelson WJ, Huie P et al. Post-ischemic injury, delayed function and Na+/K(+)-ATPase distribution in the transplanted kidney. Kidney Int 1995:48: 1308-1315.

29. Kwon O, Nelson WJ, Sibley R et al. Backleak, tight junctions, and cell- cell adhesion in postischemic injury to the renal allograft. J Clin Invest 1998:101:2054-2064.

30. Kahan B, Mickey R, Flechner SM et al. Multivariate Analysis of risk factors impacting on immediate and eventual cadaver allograft survival in cyclosporine-treated recipients. Transplantation 1987:43:65-70.

31. Troppmann C, Gillingham KJ, Gruessner RWG et al. Delayed graft function in the absence of rejection has no long-term impact. Transplantation 1996:61:1331-1337.

32. Lechevallier E, Dussol B, Luccioni A et al. Posttransplantation acute tubular necrosis: Risk factors and implications for graft survival. Am J Kidney Dis 1998:32: 984-991 


\section{CALCIUM LEVELS AS A RISK FACTOR FOR DELAYED GRAFT FUNCTION}

Henk Boom, Marko J. K. Mallat, Johan W. de Fijter, Leendert C. Paul, Jan A. Bruijn and Leendert A. van Es

Transplantation 2004; 77(6): 868-873 
Background Delayed graft function (DGF) occurs in upto $50 \%$ of renal transplants. Hypercalcemia and hyperparathyroidism are associated with impaired renal function. Little is known on the effects of serum calcium levels on delayed graft function. This issue was addressed in the current study.

Methods Patients receiving a cadaveric renal transplant between 1986-1996 were studied. Data on calcium metabolism and histological characteristics of nephrocalcinosis, acute tubular necrosis (ATN) and acute rejection in biopsies taken within the first week, were related to the occurrence of DGF.

Results The incidence of DGF in a cohort of 585 cadaveric transplants was 31\%. DGF correlated independently with serum calcium levels (OR 1.14 (95\% Cl 1.04-1.26) per $0.1 \mathrm{mmol} /$ I). The use of calcium channel blockers before transplantation protected against DGF (OR 0.5 ( $95 \% \mathrm{Cl} 0.29-0.87)$. In this selected group we found an association with histological signs of ATN and DGF. However, most of the biopsies also had features of acute rejection or nephrocalcinosis. Nephrocalcinosis was found in 12 of 71 biopsies and was not associated with serum calcium levels or the occurrence of DGF.

Conclusions In this study, serum calcium levels were independently associated with DGF. This could not be explained by the presence of microscopic nephrocalcinosis. Therefore DGF is attributed to high intracellular calcium levels. As calcium supplementation and vitamin $D$ analogues are commonly used in dialysis practice, hypercalcemia influences longterm graft outcome by its effect on DGF. The pretransplant use of calcium channel blockers has a protective effect on the occurrence of DGF. 


\section{INTRODUCTION}

The pathogenesis of acute tubular necrosis (ATN) in human kidneys remains enigmatic despite great scientific interest and investigative efforts. The poor correlation between the clinical occurrence of acute renal failure and the morphological manifestations of ATN in the renal biopsy has hampered progress in this field (1). The regular occurrence of delayed graft function (DGF) immediately following transplantation of postmortal kidneys has renewed this interest. However, the circumstances in which DGF occurs in renal allografts differ from the conditions in which acute renal failure develops in native kidneys. Conditions during explantation and implantation of the donor organ as well as calcineurin inhibitor toxicity and rejection episodes immediately following transplantation may be responsible for or contribute to the development of DGF (2). The interest in the occurrence of DGF was heightened by the finding that rejection episodes are more likely to occur in association with DGF $(3,4)$. In combination with rejection episodes DGF also influences the long term prognosis of graft function $(4,5)$.

The study of the morphological manifestations of DGF in renal allografts is facilitated by the fact that graft biopsies are more readily obtained than biopsies from native kidneys. It is conceivable that the discrepancy between DGF and ATN in allografts is not only explained by factors related to the transplantation procedure, including calcineurine toxicity, but also by elevated serum phosphate - and / or serum calcium levels at the time of transplantation.

The mechanism by which hypercalcemia causes acute renal failure remains largely hypothetical. In dogs and rats, acute renal failure has been reported as a result of experimentally induced hypercalcemia (6). In animal models 3 types of nephrocalcinosis are distinguished: chemical nephrocalcinosis, microscopic nephrocalcinosis and macroscopic nephrocalcinosis (7). The latter is characterized by gross calcium deposits found on radiographic investigations. Microscopic nephrocalcinosis is characterized by microscopic calcium deposits, mainly located in the lumen of the tubules. Its effect on renal function is thought to be caused by tubular obstruction and tubular back-leak. Chemical nephrocalcinosis, assumed to be present when macroscopic and microscopic nephrocalcinosis are excluded, affects glomerular filtration rate by vasoconstriction and natriuresis induced volume constriction (8). Calcium is a co-factor in the activation of proteolytic enzymes that are linked with tubular integrity. Several factors have been described to play a role in the development of tubular damage caused by oxygen deprivation such as abnormal calcium homeostasis, reactive oxygen species and activation of enzymes (9). Hypoxia has been found to reduce the cellular pool of adenosine triphosphate (ATP) leading initially to accumulation of adenosine di- and monophosphate and further catabolism to hypoxanthine and xanthine and the generation of reactive oxygen species (10). As a consequence of ATP depletion, cells are no longer able to extrude calcium. The influx of calcium occurs predominantly during reperfusion and reoxygenation. High cytosolic concentration of free calcium has been shown to activate calcium-dependent enzymes such as phospholipases, nucleases, and cysteine proteases (11). The wide spread use of vitamin $D$ analogues in dialysis patients and the experimental evidence for a nephrotoxic effect of hypercalcemia prompted us to investigate the role of serum calcium in the occurrence of DGF. 


\section{METHODS}

\section{Patients}

Patients who received a cadaveric renal transplant between January 1986 and December 1996 were included in this study. Twenty-six patients experiencing primary non-function (PNF) were excluded from the study. PNF was defined as grafts that never functioned and led to transplantectomy. A total of 585 transplants were analyzed. DGF was defined when serum creatinine levels increased, remained unchanged or decreased less than $10 \%$ per day in three consecutive days in the first week after transplantation. Primary function (PF) was present when they did not meet the definition of DGF and acute rejection was excluded. All patients that received anti-rejection treatment in the first week were categorized as primary function and acute rejection. Cold ischemia time (CIT) was calculated from the Eurotransplant report; warm ischemia time (WIT) was registered by the attending transplant physician. Only three patients received a graft from a non heart beating donor.

The standard immunosuppressive regimen consisted of prednisone and Cyclosporine-A (Sandimmune ${ }^{\circledR}$ soft gelatin capsules). CsA was administered intravenously in a dose of 3 $\mathrm{mg} / \mathrm{kg} /$ day for the first 48 hours, starting at the onset of surgery. The initial oral dose of CsA was $8-10 \mathrm{mg} / \mathrm{kg} /$ day from day 2 onward, divided in three daily doses and subsequently tapered. Doses were adjusted according to CsA trough level monitoring. All patients received $20 \mathrm{mg}$ of prednisone starting on day one. Rejection episodes were treated with 1 gram of methylprednisolone intravenously for 3 days or rabbit anti-thymocyte globulin for 10 days, as described previously (12).

\section{Calcium, phosphate, parathormone (PTH)}

Concentrations of calcium, phosphate, parathormone (PTH) and albumin were determined on serum samples, obtained on admission for transplantation. Calcium values were corrected for protein binding. Intact PTH was measured with a two-side radioimmunometric assay (IRMA) from Nichols Institute, San Juan Capistrano CA, USA.

\section{Histological examination}

During the study period 85 biopsies were taken within 7 days after transplantation. Fourteen biopsies were excluded because sufficient material was not available for analysis. A total of 71 biopsies could be studied. Transplantectomy specimens were not included in the analysis. Needle biopsies were taken to detect or exclude early graft rejection, not to confirm ATN. Three wedge biopsies were taken during surgical re-interventions.

Kidney sections stained by hematoxylin-eosin (HE), periodic acid-Schiff (PAS) and silvermethenamine were scored for manifestations of ATN, according to Olsen et al (13). Signs and severity of rejection in allograft biopsies were taken from the light microscopic description of these biopsies in the clinical records. Acute rejection episodes were retrospectively classified based on the criteria described in the Banff 1997 classification of renal allograft pathology (14). 
In order to detect calcium deposits, paraffin sections were stained according to Von Kossa (15) and scored independently by JAB and LES. Nephrocalcinosis was defined when Von Kossa positive material was seen at the same location in at least two consecutive sections (fig.2).

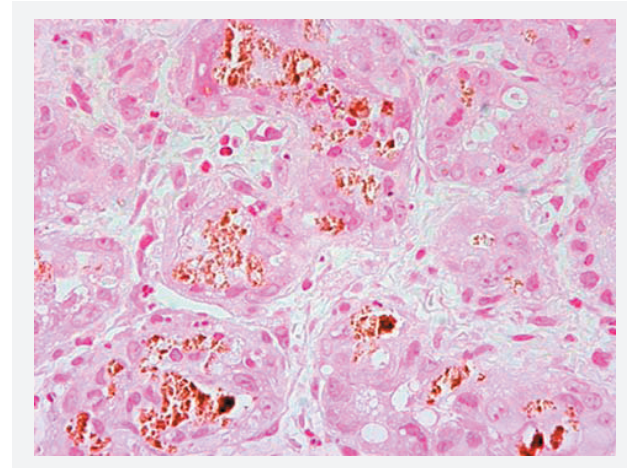

\section{Figure 2}

Representative slide showing

nephrocalcinosis (Von Kossa staining).

\section{Statistical analysis}

Characteristics among groups were compared using Student's t-test for independent continuous variables and Pearson chi-square test for categorical variables. Binary logistic regression analysis was used to assess the risk for occurrence of DGF for each variable separately. Multivariate binary logistic regression was used to adjust for confounding. All analyses were performed using SPSS statistical software package (version 10.07;SPSS Inc. Chicago III.)

\section{RESULTS}

DGF occurred in 183 (31\%) of the 585 patients studied (Table 1). Sex of either donor or recipient did not correlate with DGF, but a higher donor age correlated significantly ( $p=$ 0.0005 ) with the occurrence of DGF, whereas recipient age had no influence. Warm ischemia time (WIT) was longer for patients with DGF, but this difference was not significant, whereas cold ischemia time (CIT) was significantly longer in recipients with DGF. Recipients who used calcium channel entry blockers before transplantation experienced less frequent $\operatorname{DGF}(p=0.01)$.

\section{Calcium, phosphate and PTH}

Recipients with DGF had significantly higher total serum calcium $(p=0.001)$ and albumen corrected calcium levels $(p=0.01$ ) than recipients without DGF (Table 1$)$. In a logistic regression model in which several risk factors for the occurrence of DGF were included the Odds Ratio for serum albumen corrected serum calcium was 1.14 for each $0.1 \mathrm{mmol} / \mathrm{l}$ increase of serum calcium. When serum calcium levels were stratified, calcium levels of $2.75 \mathrm{mmol} / \mathrm{l}$ or higher were associated with a higher occurrence of DGF compared to levels of less than 2.55 
$\mathrm{mmol} / \mathrm{I}$ (OR: $2.51 ; 95 \% \mathrm{Cl}: 1.59$ - 3.98; $\mathrm{p}<0.0005)$ (Table 2). However, the individual calcium levels of recipients with and without DGF shows a considerable overlap (fig.1). The relation between corrected calcium levels and the predicted probability of DGF did not show a critical value above which DGF was more likely to occur. Serum phosphate levels did not correlate with the occurrence of DGF and neither did the calcium phosphate product or the albumin corrected calcium-phosphate product. Serum PTH levels were higher in recipients with DGF, but this difference was not significant $(p=0.81)$.

Table 1: Transplantation characteristics and of calcium and phosphate metabolism of 585 kidney

\begin{tabular}{|c|c|c|c|c|}
\hline & $\begin{array}{l}\text { Total } \\
\mathrm{n}=585\end{array}$ & $\begin{array}{l}\mathrm{PF} \\
\mathrm{n}=402\end{array}$ & $\begin{array}{l}\text { DGF } \\
\mathrm{n}=183\end{array}$ & P-value \\
\hline \multicolumn{5}{|l|}{ Transplantation characteristics } \\
\hline Sex recipient male (\%) & 63 & 62 & 66 & 0.34 \\
\hline Sex donor male (\%) & 58 & 59 & 56 & 0.66 \\
\hline Recipient age (years) & $46.2(12.8)$ & $45.9(12.5)$ & $47.1(13.5)$ & 0.68 \\
\hline Donor age (years) & $38.4(15.0)$ & $36.7(14.6)$ & $42.3(15.2)$ & $<0.0005$ \\
\hline Number of transplants & $1.20(0.48)$ & $1.22(0.49)$ & $1.17(0.46)$ & 0.34 \\
\hline Pre-Tx CCB's (\%) & 17.5 & 20 & 11.4 & 0.01 \\
\hline CIT (hours) & $28.7(6.7)$ & $28.0(6.6)$ & $30.1(6.7)$ & $<0.00001$ \\
\hline \multicolumn{5}{|l|}{ Pre-Tx dialysis mode } \\
\hline Time on dialysis (months) & $40.5(69)$ & $43(80)$ & $35(31)$ & 0.61 \\
\hline Preservation fluid (\% EC) & 41 & 40 & 43 & 0.62 \\
\hline WIT (minutes) & $27.8(9.3)$ & $27.5(9.1)$ & $28.4(9.6)$ & 0.58 \\
\hline \multicolumn{5}{|l|}{ Laboratory parameters } \\
\hline Serum calcium (mmol/l) & $2.57(0.24)$ & $2.54(0.24)$ & $2.63(0.25)$ & $<0.001$ \\
\hline Corrected serum calcium (mmol/l) & $2.47(0.22)$ & $2.46(0.22)$ & $2.51(0.21)$ & 0.01 \\
\hline Serum phosphate (mmol/l) & $1.67(0.51)$ & $1.68(0.50)$ & $1.66(0.52)$ & 0.72 \\
\hline Calcium*Phosphate product & $4.27(1.28)$ & $4.23(1.24)$ & $4.34(1.35)$ & 0.39 \\
\hline $\begin{array}{l}\text { Corrected Calcium* Phosphate } \\
\text { product }\end{array}$ & $4.11(1.26)$ & $4.10(1.23)$ & $4.14(1.33)$ & 0.72 \\
\hline Serum PTH (pmol/l)* & $18.5(23.6)$ & $18.3(24.8)$ & $19.1(21.1)$ & 0.81 \\
\hline
\end{tabular}

*Since February 1993 ( $\mathrm{n}=179) ;$ () mean (SD) () Mean (SD);CCB's : calcium channel blockers; EC: Euro Collins 
Calcium levels as a risk factor for delayed graft function

Table 2: Independent risk factors for DGF in the cohort 585 patients transplanted in the period 1986 - 1996

\begin{tabular}{llll}
\hline & OR & $95 \% \mathrm{Cl}$ & p-value \\
\hline Adjusted serum calcium & & & \\
$\quad<2.54$ & 1 & & \\
$2.55-2.74$ & 1.54 & $0.98-2.43$ & 0.062 \\
$>2.74$ & 2.51 & $1.59-3.98$ & $<0.0005$ \\
$\mathrm{CIT}>28 \mathrm{~h}$ & 1.70 & $1.16-2.48$ & 0.007 \\
Donor age $>=50$ years & 1.48 & $0.975-2.23$ & 0.07 \\
Use of $\mathrm{CCB}$ & 0.49 & $0.28-0.85$ & 0.01 \\
\hline
\end{tabular}

${ }^{*}$ CCB: Calcium Channel Blocker

\section{Figure 1}

The distribution of albumen corrected serum calcium levels, showing a considerable overlap between patients with PF and DGF
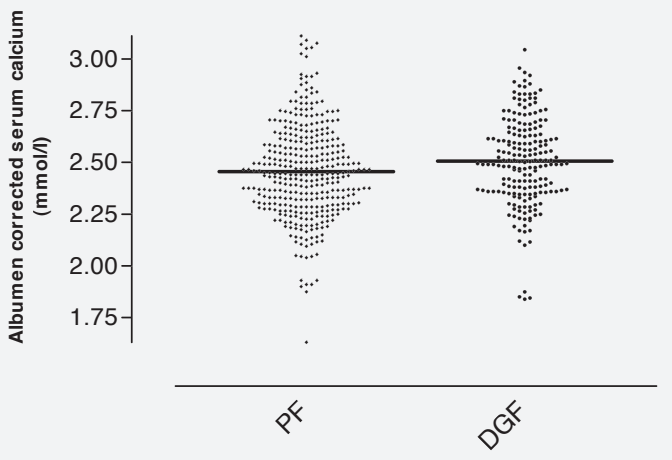

\section{Histological examination}

To determine whether the results could have been influenced by the biopsy strategy used to take graft biopsies, characteristics of biopsied $(n=85)$ and non-biopsied $(n=500)$ patients were compared (Table 3). No differences were found, except for the serum phosphate levels $(p=0.05)$. Despite the significant higher phosphate levels in the biopsied patients, hyperphosphatemia was not associated with DGF.

In a total of 71 biopsies, 34 were taken from patients with DGF and 37 were taken from patients with PF.DGF correlated significantly with the presence of ATN in the renal biopsies (Table 4A). However most biopsies, except six had other morphological changes such as acute rejection or nephrocalcinosis. The association of these changes with DGF was therefore analyzed (Table 4B and 4C). DGF did not correlate with histological signs of acute rejection, except when it was accompanied with ATN (Table 4B). Nephrocalcinosis did not correlate with DGF, but it could have been associated with other histological abnormalities. It did not occur in the absence of other abnormalities like acute rejection or ATN (Table 4C). 
Table 3: Characteristics of 585 patients with an allograft biopsy or no biopsy within 7 days after transplantation

\begin{tabular}{llll}
\hline & Biopsy & No biopsy & p-value \\
& $\mathrm{n}=85$ & $\mathrm{n}=500$ & \\
\hline Sex recipient male (\%) & 65 & 63 & 0.82 \\
Sex donor male (\%) & 57 & 59 & 0.91 \\
Age recipient & $44(13)$ & $47(13)$ & 0.14 \\
Age donor & $38(15)$ & $39(15)$ & 0.58 \\
CIT (hours) & $23.0(6.6)$ & $28.6(6.8)$ & 0.67 \\
WIT (min.) & $26.8(8.5)$ & $28.0(9.4)$ & 0.26 \\
DGF & $29(36 \%)$ & $178(36 \%)$ & 0.94 \\
Use of CCB's (\%) & 18 & 17 & 0.77 \\
Serum calcium (mmol/l) & $2.54(0.22)$ & $2.58(0.25)$ & 0.21 \\
Corrected Calcium (mmol/l) & $2.44(0.22)$ & $2.48(0.22)$ & 0.11 \\
Serum phosphate (mmol/l) & $1.77(0.49)$ & $1.65(0.51)$ & 0.05 \\
Calcium / phospate product & $4.47(1.24)$ & $4.23(1.28)$ & 0.12 \\
Calcium / phospate product (albumen corr.) & $4.32(1.28)$ & $4.07(1.26)$ & 0.10 \\
PTH (pmol/l ) * & $25.9(32.2)$ & $17.3(21.0)$ & 0.16 \\
\hline
\end{tabular}

() = mean (SD); *since February 1993 ( $N=179)$; CCB: Calcium channel blocker

Table 4 A: Histological characteristics of ATN and initial graft function, showing a relation between the presence of ATN and DGF, especially in combination with signs of acute rejection or nephrocalcinosis

\begin{tabular}{llll}
\hline & $\begin{array}{l}\text { PF } \\
\mathrm{n}=37\end{array}$ & $\begin{array}{l}\text { DGF } \\
\mathrm{n}=34\end{array}$ & p-value \\
\hline No ATN & $33(89)$ & $19(56)$ & 0.002 \\
\hline ATN total & $4(11)$ & $15(44)$ & 0.002 \\
\hline ATN only & $2(5)$ & $6(18)$ & NS \\
ATN + rejection & $2(5)$ & $4(12)$ & 0.04 \\
ATN + NC* & 0 & $5(18)$ & 0.001 \\
\hline
\end{tabular}

* NC: nephrocalcinosis; $(\%)$

Table 4 B: Histological characteristics of acute rejection and initial graft function, showing a lack of correlation in the absence of signs of ATN.

\begin{tabular}{llll}
\hline & $\begin{array}{l}\text { PF } \\
\mathrm{N}=37\end{array}$ & $\begin{array}{l}\text { DGF } \\
\mathrm{n}=34\end{array}$ & p-value \\
\hline No rejection & $11(30)$ & $14(41)$ & NS \\
\hline Total rejection & $26(70)$ & $20(59)$ & NS \\
\hline Rejection only & $21(57)$ & $12(35)$ & NS \\
Rejection + ATN & $2(5)$ & $4(12)$ & 0.04 \\
Rejection + NC* & $3(7)$ & $4(12)$ & NS \\
\hline
\end{tabular}

* NC: nephrocalcinosis; (\%) 
Table 4 C: Histological characteristics of nephrocalcinosis and initial graft function, showing a lack of correlation in the absence of signs of ATN.

\begin{tabular}{llll}
\hline & $\begin{array}{l}\text { PF } \\
\mathrm{n}=37\end{array}$ & $\begin{array}{l}\text { DGF } \\
\mathrm{n}=34\end{array}$ & p-value \\
\hline No NC & $34(92)$ & $25(73)$ & 0.05 \\
\hline Total NC & $3(11)$ & $9(27)$ & 0.05 \\
\hline NC only & 0 & 0 & \\
NC + ATN & 0 & $5(15)$ & 0.001 \\
NC + Rejection & $3(7)$ & $4(12)$ & NS \\
\hline
\end{tabular}

NC: nephrocalcinosis; (\%)

\section{Role of calcineurin inhibitors on DGF}

In the current study with the use of continuous infusion of Cyclosporin all patients received a standardized systemic exposure to cyclosporin in the first postoperative days. To asses a possible confounding effect of this intravenous regimen, we extended our study with a cohort of patients transplanted between 1997 and 2002, who received triple immunosuppressive therapy with the micro-emulsion formula of cyclosporin (Neoral $\left.{ }^{\circledR}\right)$, Prednison and Mycophenolate Mofetil (CellCept ${ }^{\circledR}$ ). The oral doses of cyclosporine were adjusted according to 12 hours serum trough level monitoring, aiming at $250-350 \mu \mathrm{g} / \mathrm{l}$. Fifty five out of 222 patients (25\%) experienced DGF. Cold ischemia time (CIT) and donor age were again significantly associated with DGF. Also in this cohort, the increased pretransplant serum calcium levels were associated with the occurrence of DGF (PF vs. DGF: $2.49 \mathrm{mmol} / \mathrm{l} \mathrm{vs.} 2.54$ $\mathrm{mmol} / \mathrm{l} ; \mathrm{p}: 0.11)$. The results of the multivariate analysis are shown in table 5. The increased serum calcium levels were associated with an increased occurrence of DGF (OR: 3.12: calcium $>2.75 \mathrm{mmol} / \mathrm{l}$ vs. serum calcium $<2.55 \mathrm{mmol} / \mathrm{l} ; 95 \% \mathrm{Cl}: 1.18-8.26 ; \mathrm{p}: 0.02$ ). Patients who used calcium channel blockers prior to and at the time of transplantation were protected against DGF (OR $0.57 ; 95 \% \mathrm{Cl}: 0.26-1.23 ; \mathrm{p}=0.15)$, although no statistical significance was reached, probably because of the limited number of patients that could be analyzed in this additional cohort of patients (Table 5).

Table 5: Independent risk factors for DGF in the cohort 222 patients transplanted in the period 1997 - 2002

\begin{tabular}{llll}
\hline & OR & $95 \% \mathrm{Cl}$ & p-value \\
\hline Serum calcium & & & \\
$<2.55 \mathrm{mmol} / \mathrm{I}$ & 1 & & \\
$2.55-2.74 \mathrm{mmol} / \mathrm{I}$ & 1.60 & $0.73-3.51$ & 0.24 \\
$>2.75 \mathrm{mmol} / \mathrm{I}$ & 3.12 & $1.18-8.26$ & 0.02 \\
$\mathrm{CIT}>28 \mathrm{~h}$ & 2.00 & $0.93-4.30$ & 0.07 \\
Donor age $>50$ yrs & 3.49 & $1.71-7.11$ & 0.001 \\
Use of CCB & 0.57 & $0.26-1.24$ & 0.16 \\
\hline
\end{tabular}

${ }^{*}$ CCB: Calcium Channel Blocker 


\section{DISCUSSION}

In this retrospective study, the occurrence of DGF was 35\%. We also found that among other known risk factors, like donor age and CIT, pre-transplant serum calcium level was independently associated with DGF. Although the differences between the two groups seem to be small $(0.09 \mathrm{mmol} / \mathrm{l}$ in serum calcium levels and $0.05 \mathrm{mmol} / \mathrm{l}$ for the albumen corrected serum calcium levels), the risk of DGF increased $14 \%$, with each $0.1 \mathrm{mmol} / \mathrm{l}$ rise in serum calcium level (OR 1.14 [95\%Cl: $1.04-1.26]$ ). Dialysis mode, duration of dialysis before transplantation and the preservation fluid used on the other hand did not correlate with DGF. When a commonly used definition for DGF (e.g. the need of at least 1 dialysis treatment in the first week), was used similar results were obtained. However the need of dialysis treatment in the first week also includes patients that are dialyzed for other reasons than DGF (16). In our study 12 out of 358 patients (3.4\%) were dialyzed in the first week after transplantation for other reasons than DGF.

Also the possibility of calcineurin toxicity as a risk factor for the occurrence of DGF was considered. Although histological proof of acute cyclosporin toxicity was only found in 2 biopsies in the PF group, we cannot exclude a role of cyclosporin in the development of DGF. However differences in cyclosporin assays over time and the high inter patient variability of systemic exposure to cyclosporin preclude any meaningful retrospective evaluation of systemic exposure. $(17,18)$. To asses a possible confounding effect of this intravenous regimen, we analyzed an other group of patient that was transplanted in the period 1997-2002 and that were treated with $\mathrm{Neora}^{\circledR}$ soft gelatin capsules twice daily, assuming that the occurrence of high serum levels is limited. Again albumen corrected serum calcium levels tend to be higher in the DGF group (OR 3.12; p: 0.02 calcium $>2.75 \mathrm{mmol} / \mathrm{l} \mathrm{vs} .<2.55 \mathrm{mmol} / \mathrm{l}$ ). We also found that the use of calcium channel blockers (CCB) prior to the transplantation protected against the occurrence of DGF. Unfortunately the level of significance was not reach, probably because of the limited number of patients that could be analyzed.

No relation was found between microscopic nephrocalcinosis and serum calcium levels ( $2.43 \mathrm{mmol} / \mathrm{l}$ in each group). Acute renal failure in native kidneys has been reported in patients with serum calcium levels above $3.5 \mathrm{~mol} / \mathrm{l}$. In native kidneys, these conditions occur in the milk alkali syndrome (19), severe hyperparathyroidism, PTH-related proteins (PTHrP) associated conditions(20), multiple myeloma (21) or vitamin D intoxication (22). Although the corrected serum calcium exceeded the serum level of $2.7 \mathrm{mmol} / \mathrm{l}$ in only $19 \%$ of the cases, we found an effect of serum calcium levels on initial graft function. Since we did not find a critical value above which DGF occurred more often, it is difficult to predict DGF on the basis of serum calcium levels alone.

Little is known about the effects of hypercalcemia on the initial graft function. Torregosa et al. (23) reported a significant effect of elevated PTH levels on the incidence of DGF, whereas serum Vitamin D levels and serum calcium levels did not differ. Therefore we analyzed the pre-transplant PTH-levels that were routinely measured; 179 pre-transplant PTH levels were measured but no significant differences were found between the two groups (PF vs. DGF: $18.3 \mathrm{pmol} / \mathrm{lvs} .19 .1 \mathrm{pmol} / \mathrm{l} ; \mathrm{p}=0.81$ ).

Vitamin $\mathrm{D}$ analogues and calcium-containing phosphate binders are prescribed on a regular basis in patients that are on renal replacement therapy to prevent renal bone disease. 
Serum calcium levels and the total body calcium load therefore will rise. This is associated with a higher incidence of calcifications of the coronary arteries and possibly with inferior patient survival $(24,25)$. Since DGF is associated with inferior long-term graft outcome (26) this prophylactic regimen may not only influence patient survival but also graft survival. Unfortunately Vitamin D levels were not routinely measured in our study. A prospective study is required to determine the risk of developing DGF as a consequence of calcium and vitamin $D$ supplements in transplant recipients.

Of the 26 biopsied patients with DGF only 13 biopsies showed signs of ATN whereas in the other 13 biopsies no signs of ATN were observed. Therefore attempts were made to find other morphological explanations for DGF in these patients. ATN was only associated with DGF when it coincided with acute rejection or nephrocalcinosis. Acute rejection and nephrocalcinosis alone or in combination with other histological features than ATN were not associated with DGF. This means that DGF and ATN are firmly correlated in this cohort. In contrast to the whole group of patients, we found no differences in serum calcium levels between the PF and DGF group that had renal biopsies in the first week $(p=0.3)$.

This study can not explain the effects of high pre-transplant serum calcium levels on initial graft function by the development of calcium depositions. Very few clinical studies have looked at the morphological substrate of hypercalcemia in the kidney. Prospective studies have not been performed and on the basis of the relative benign outcome of DGF it will be hard to justify protocolized prospective renal biopsies in recipients with and without DGF. In animal models 3 types of nephrocalcinosis can be distinguished: chemical nephrocalcinosis, microscopic nephrocalcinosis and macroscopic nephrocalcinosis (7). The latter is characterized by gross calcium deposits found on radiographic investigations. As macroscopic nephrocalcinosis is not present in this study, microscopic and chemical nephrocalcinosis might explain the effect of high serum calcium levels on initial graft function. Microscopic nephrocalcinosis is characterized by microscopic calcium deposits, mainly located in the lumen of the tubules. It is supposed to be a transitional phase between chemical and macroscopic nephrocalcinosis. Microscopic nephrocalcinosis is associated with increased calcium $x$ phosphate product and with chronic renal failure. Especially high serum phosphate levels seem to trigger this process. Its effect on renal function is thought to be caused by tubular obstruction and tubular back-leak. Chemical nephrocalcinosis, assumed when macroscopic and microscopic nephrocalcinosis are excluded, affects glomerular filtration rate by vasoconstriction and natriuresis induced volume constriction (8) and is histological characterized by areas of focal necrosis in the distal tubules and medullary collecting duct. High calcium content of the medullary area was found and the functional substrate was characterized by impaired function of the distal tubules. The role of cytoplasmatic calcium as an intracellular messenger in many important cell functions might explain these functional changes associated with the high cytoplasmatic calcium content. Calcium dependent enzymes that were analyzed in vitro in this respect are the cystein proteases, like calpaine and the caspases (27). In this study, we found a correlation of DGF with serum calcium levels but not with calcium deposits. This suggests that chemical nephrocalcinosis rather than microscopic nephrocalcinosis is the underlying cause of the effect of serum calcium levels on initial graft function.

In this context our finding that the use of pre-transplant calcium channel blockers (CCB's) protects against DGF is interesting. CCB's are reported to ameliorate initial graft function 
(28). The mechanisms by which CCB's have this protective effect may be related to an increase of renal perfusion because of vasodilatation of the glomerular arterioles (29). On the other hand it is conceivable that the CCB induced reduction of calcium influx into tubular cells during ischemic and reperfusion periods and thereby reduce the generation of oxygen-free radicals and activation of cystein proteases (11).

In this study we found that serum calcium levels were independently correlated with the occurrence of DGF. In contrast to other studies a significant correlation was found between DGF and ATN, but not with microscopic nephrocalcinosis. Therefore DGF should be attributed to high intracellular calcium levels (chemical nephrocalcinosis). As calcium supplementation and vitamin $\mathrm{D}$ analogues are commonly used in dialysis practice, hypercalcemia might cause a higher incidence of DGF and therefore influence long-term outcome of renal transplantation. The use of calcium channel blockers protects against the occurrence of DGF. 


\section{REFERENCES}

1. Rosen S, Heyman SN. Difficulties in understanding human "acute tubular necrosis": limited data and flawed animal models. Kidney Int 2001:60: 1220-1224.

2. Lechevallier E, Dussol B, Luccioni $A$ et al. Posttransplantation acute tubular necrosis: Risk factors and implications for graft survival. Amer J Kidney Dis 1998:32: 984-991.

3. Howard R.J., Pfaff W.W., Brunson M.E. et al. Increased incidence of rejection in patients with delayed graft function. Clinical Transplantation 1994:8: 527-531.

4. Shoskes DA, Cecka JM. Deleterious effects of delayed graft function in cadaveric renal transplant recipients independent of acute rejection. Transplantation 1998:66: 1697-1701.

5. Troppmann C, Gillingham KJ, Gruessner RWG et al. Delayed graft function in the absence of rejection has no long-term impact. Transplantation 1996:61:1331-1337.

6. Epstein FH. Calcium and the kidney. Am J Med 1968:45: 700-714.

7. Wrong O.Nephrocalcinosis. In:Davidsen AM, Cameron JS, Grunfeld JP, Kerr DNS, Ritz E, Winearls CG, eds. Oxford Textbook of Clinical Nephrology. Oxford, New York,Tokyo: Oxford University Press, 1998: 13751396.

8. Levi M, Ellis MA, Berl T. Control of renal hemodynamics and glomerular filtration rate in chronic hypercalcemia. Role of prostaglandins, renin-angiotensin system, and calcium. J Clin Invest 1983:71: 16241632.

9. Cheung J.Y., Bonventre J.V., Malis Ch.D., Leaf A. Calcium and Ischemic Injury. New England Journal of Medicine 1986:314: 1670-1675.

10. Takeda M, Shirato I, Kobayashi M, Endou H. Hydrogen peroxide induces necrosis, apoptosis, oncosis and apoptotic oncosis of mouse terminal proximal straight tubule cells. Nephron 1999:81:234-238.

11. Edelstein CL, Shi Y, Schrier RW. Role of caspases in hypoxia-induced necrosis of rat renal proximal tubules. J Am Soc Nephrol 1999:10: 1940-1949.

12. Johan de Fijter, Marko JK Mallat, Ilias N Doxiadis, Jan Ringers, Frits R Rosendaal, Frans HJ Claas, and Leendert C Paul. Increased immunogenicity and cause of graft loss of old donor kidneys. J Am Soc Nephrol July, 2001; 12 (7) 2001.

13. Olsen S, Burdick JF, Keown PA, Wallace AC, Racusen LC, Solez K. Primary acute renal failure („, acute tubular necrosis") in the transplanted kidney:morphology and pathogenesis. Medicine (Baltimore) 1989:68: 173-187.

14. Racusen LC, Solez K, Colvin RB et al. The Banff 97 working classification of renal allograft pathology. Kidney Int 1999:55: 713-723.

15. Von Kossa, J. Uber die in organismus kunstlich erzeugbaren Verkalkungen. Beitr.Pathol.Anat.Allg.Pathol. 29 1901; 163-202.

16. Giralclasse $M$, Hourmant $M$, Cantarovich $D$ et al. Delayed graft function of more than six days strongly decreases long-term survival of transplanted kidneys. Kidney Int 1998:54:972-978.

17. Cremers SC, Scholten EM, Schoemaker RC et al. A compartmental pharmacokinetic model of cyclosporin and its predictive performance after Bayesian estimation in kidney and simultaneous pancreaskidney transplant recipients. Nephrol Dial Transplant 2003:18: 1201-1208.

18. Kovarik JM, Mueller EA, van Bree JB, Arns W, Renner E, Kutz K. Within-day consistency in cyclosporine pharmacokinetics from a microemulsion formulation in renal transplant patients. Ther Drug Monit 1994:16: 232-237.

19. Abreo K, Adlakha A, Kilpatrick S, Flanagan R, Webb R, Shakamuri S. The milk-alkali syndrome. A reversi- 
ble form of acute renal failure. Arch Intern Med 1993:153: 1005-1010.

20. Abraham P, Ralston SH, Hewison M, Fraser WD, Bevan JS. Presentation of a PTHrP-secreting pancreatic neuroendocrine tumour, with hypercalcaemic crisis, pre-eclampsia, and renal failure. Postgrad Med J 2002:78: 752-753.

21. Rota S, Mougenot B, Baudouin B et al. Multiple myeloma and severe renal failure: a clinicopathologic study of outcome and prognosis in 34 patients. Medicine (Baltimore) 1987:66: 126-137.

22. Zawada ET, Jr., Sanderson EW, Rossing D, Ohrt D, Simmons J. Hypercalcemia and acute renal insufficiency in a 24-year-old white male with lung disease. Am J Nephrol 1986:6: 152-157.

23. Torregosa, Campistol, Fenollasa, Montesinos, Romar, Martinez de Osaba. Secondary Hyperparathyroidism and Post-Transplant Acute Tubular Necrosis. Nephron 1996:73:67-72.

24. Chertow GM, Burke SK, Raggi P. Sevelamer attenuates the progression of coronary and aortic calcification in hemodialysis patients. Kidney Int 2002:62: 245-252.

25. Raggi P, Boulay A, Chasan-Taber $\mathrm{S}$ et al. Cardiac calcification in adult hemodialysis patients. A link between end-stage renal disease and cardiovascular disease? J Am Coll Cardiol 2002:39:695-701.

26. Boom H, Mallat MJ, De Fijter JW, Zwinderman AH, Paul LC. Delayed graft function influences renal function, but not survival. Kidney Int 2000:58: 859-866.

27. Edelstein, C. L., Ling H., and Schrier, R.W.The Nature of renal cell injury. Kidney Int 51 1997; 1341-1351.

28. Epstein M. Calcium antagonists and the kidney. Implications for renal protection. Am J Hypertens 1991:4: 482S-486S.

29. Epstein A, Beall A, Wynn J, Mulloy L, Brophy CM. Cyclosporine, but not FK506, selectively induces renal and coronary artery smooth muscle contraction. Surgery 1998:123:456-460. 


\section{THE EXPRESSION OF CASPASE-3}

AND MANGANESE SOD IN DISTAL TUBULES PREDICTS POST -TRANSPLANT ACUTE TUBULAR NECROSIS OR DGF

Henk Boom; Emile de Heer; Annemieke van der Wal; Laurens Kruidenier; Johan W. de Fijter; Hallgrimur Benediktsson; Leendert C. Paul and Leendert A. van Es

Submitted 
Background Acute tubular necrosis (ATN) in renal allograft biopsies correlates poorly with delayed graft function (DGF). Factors involved in the pathogenesis of DGF were evaluated in biopsies in an attempt to refine the recognition of DGF.

Methods Of a total of 85 biopsies taken within the first week after transplantation, 41 biopsies were suitable for this study: ten from patients with DGF, and 31 from patients without DGF. Anti-cubulin and anti-AE-1/AE-3 antibodies were used to identify proximal and distal tubules respectively. The TUNEL technique and staining for active caspase-3 were used to demonstrate apoptosis. Antibodies against three types of super oxide dismutase (SOD) were used as markers of the protective tubular response. Tubular regeneration was evaluated using anti-ki 67 and anti-vimentin antibodies.

Results DGF occurred in $24 \%$ of the transplant recipients. ATN coincided with DGF in $31 \%$ of the cases. The predictive value of finding ATN in the biopsy of a graft with DGF was only $50 \%$. Absence of distal caspase- 3 staining predicted the absence of ATN in $78 \%$ of cases. The presence of caspase- 3 predicted ATN in $75 \%$ of cases. The detection of Mn-SOD in distal tubules predicts the absence of DGF in $78 \%$ of the cases.

Conclusions The use of immuno-histochemical staining on renal biopsies improved its predictive value with respect to ATN and DGF:The absence of active caspase- 3 in distal tubular epithelium predicts the absence of ATN in $78 \%$ of cases, whereas its presence predicts ATN in $75 \%$ of cases. The presence of Mn-SOD in distal tubules predicts the absence of DGF in $78 \%$ of cases. 


\section{INTRODUCTION}

The relationship between the functional and the morphological manifestations of acute renal failure remains enigmatic. It is generally accepted that acute tubular necrosis (ATN) is the morphological expression of acute renal failure due to ischemia-reperfusion injury. However, extensive tubular necrosis is not a typical feature of ATN (1). The tubular changes in human ATN are subtle and consist of flattening of proximal tubules with loss of brush border and necrosis of individual cells with focal denudation of the tubular basement membrane (2). Acute tubular injury therefore might be a better term to describe the more subtle morphological markers as a substrate of acute renal failure. In clinical practice renal biopsies are rarely performed to confirm ATN, but rather to exclude other causes of ARF. Delayed graft function of renal transplants (DGF) has renewed the clinical and scientific interest in the histopathological characteristics of ARF, as it has been described as a possible risk factor for acute rejection (3) and for the development of chronic allograft nephropathy (4). Olsen reported, that although there are a lot of similarities between ATN in native kidneys and graft ATN, the latter shows more apoptosis and tubular necrosis, sometimes extending to whole tubular cross sections $(5,6)$. Although circumstances leading to ATN in transplants may differ from those leading to ATN in native kidneys, ischemia/reperfusion injury plays a role in both conditions.

Several mechanisms have been described in the development of acute tubular damage caused by oxygen deprivation such as abnormal calcium homeostasis, reactive oxygen species and activation of enzymes involved in the oxidative stress response. Hypoxia has been found to reduce the cellular pool of adenosine triphosphate (ATP) initially leading to accumulation of adenosine di- and monophosphate and the generation of reactive oxygen species. Moderately elevated levels of reactive oxygen species have been shown to cause apoptosis, whereas higher levels cause necrosis of proximal tubules (7). As a consequence of ATP depletion, cells are no longer able to excrete calcium. The influx of calcium occurs predominantly during reperfusion and reoxygenation. High cytosolic concentration of free calcium has been shown to activate calcium-dependent enzymes such as phospholipases, nucleases, and cysteine proteases (8). Activated cysteine proteases are responsible for the disruption of the microtubular and cytoskeletal network and are involved in apoptosis $(9,10)$ whereas administration of inhibitors of cysteine proteases reduce reperfusion injury in rat kidneys (11). Intact structural polarity of proximal tubules is vital for their function. Disruption of the cytoskeletal network has been reported to be associated with disturbed polarity, apoptosis and delayed graft function (12)

Protection against the toxic effects of reactive oxygen species is mediated by antioxidant enzymes, such as catalase, glutathione peroxidase (GPX) and superoxide dismutases (SODs). Extracellular-SOD (Ec-SOD) is mainly present in the extracellular fluid and matrix. Copper-Zinc SOD (Cu/Zn-SOD) is expressed constitutively throughout the body, whereas Manganese-SOD (Mn-SOD) is located exclusively in mitochondria (13) and is easily induced by oxidant stress and inflammatory conditions (14). The kidney has a remarkable capacity to recover from ischemic injury. Signs of regeneration and proliferation such as mitotic activity are characteristic of previous ischemic injury. Renal regeneration recapitulates certain aspects of renal development. Renal tubules are of mesenchymal origin, but they normally 
do not express vimentin, unless they are recovering from ischemia (15).

Renal biopsies are generally not taken from renal allografts to confirm ATN; they are taken to detect acute rejection in patients with DGF. Since DGF is a transient and treatable disorder, prospective studies to investigate the relationship between DGF and ATN are not likely to be performed. It is therefore extremely difficult to study ATN in human kidneys. Therefore we performed a retrospective study in order to determine whether molecular markers for cell death, protection against oxidative stress, regeneration and proliferation provide a better correlation with DGF than the histological features of ATN in human renal transplants.

\section{METHODS}

\section{Study Design}

DGF was defined as a failure of the graft to reduce serum creatinine concentration by more than $10 \%$ over three consecutive days for more than 1 week after transplantation (3). Using this definition DGF occurred in $24 \%$ of the transplantation procedures. Ninety percent of the patients were initially treated with prednisone and cyclosporine (Sandimmune) and $10 \%$ with prednisone and azathioprine. Between 1983 and 1996, 85 biopsies were taken within the first 7 days.

To be included in the study, a renal biopsy taken in the first week after transplantation had to be available. These biopsies were taken for clinical reasons to detect or exclude additional acute rejection or cyclosporin toxicity. No biopsies were done to confirm ATN. When acute rejection was not found in the biopsy and cyclosporin toxicity could be excluded on clinical data and histology, the biopsy was included in the study. Patients who had a renal biopsy taken within the first week and who fulfilled these criteria were divided into a group with DGF and a group with primary function (PF). Forty one biopsies were selected. Eleven wedge biopsies were taken during surgical re-interventions and 30 needle biopsies were taken for diagnostic purposes. These biopsies were subsequently evaluated using light microscopy for the presence of acute tubular necrosis (16).

The pathophysiology of acute tubular necrosis is generally divided in an ischemic phase, characterized by tubular cell apoptosis or necrosis, a protective response against superoxide radicals, and a recovery phase in which tubular cell regeneration is prominent (17). Biopsies were therefore stained for markers of apoptosis, using the TUNEL technique and by immunostaining for active caspase-3, as well as for Ki 67 and vimentin, markers for proliferation and regeneration respectively. Staining for Cu/Zn-SOD, EC-SOD and Mn-SOD was performed to evaluate the protective response of the graft against reactive oxygen species. Differences between the 2 groups were subsequently scored in a blinded protocol.

\section{Histopathological examination and scoring}

Biopsies were fixed in $3 \%$ formaldehyde and embedded in paraffin. Tissue sections were cut and stained with hematoxylin and eosin $(\mathrm{H}+\mathrm{E})$, Periodic acid-Schiff (PAS) and periodic acid-silver methenamine (PASM). The biopsies were reviewed by a pathologist without 
knowledge of the clinical findings (He.Be.). Each biopsy was evaluated for the following attributes: number of glomeruli present, extent of global and segmental glomerulosclerosis, interstitial inflammatory infiltrate, interstitial fibrosis, tubular atrophy, tubular cell shedding, focal denudation of the tubular basement membrane ("non-replacement phenomenon"), presence of necrotic tubular cells in the lumen (tubular necrosis), tubular cross-sectional necrosis, tubular nucleolar prominence (tubular cell "activation"), and tubular mitotic activity. A semiquantitative evaluation was applied and each characteristic scored on a scale of 0 - 3. (16). For a better analysis of the relation between these histological parameters and clinical and immuno-histochemical features, this histological score was simplified into 2 point scale The Banff Schema for transplant pathology was applied (18). A biopsy was classified as having morphologic changes characteristic of ATN, when tubular cells were necrotic or showed cross-sectional necrosis or when at least score 2 was reached for tubular regenerative changes (16).

Immunohistochemical techniques

TUNEL Paraffin sections $(4 \mu \mathrm{m})$ were mounted on Superfrost plus glass slides (MenselGlaser, Omnilabo, Breda, The Netherlands) and deparaffinized in xylol and ethanol $96 \%$ and dried overnight at $370 \mathrm{C}$. After inactivation of endogenous peroxidases with $1.2 \% \mathrm{H} 2 \mathrm{O} 2$ in methanol and hydration, sections were pretreated with blocking buffer consisting of $0.1 \mathrm{M}$ TRIS, 3\% BSA and 20\% normal calf serum for 30 minutes at room temperature. After rinsing with PBS, TUNEL mix (Roche Diagnostics, Almere, the Netherlands) was applied for only 30 minutes at $370 \mathrm{C}$. The prescribed incubation for 60 minutes resulted in our hands in unacceptable level of false positives in normal controls. The reaction was stopped with a mixture consisting of $40 \mathrm{cc} 0.75 \mathrm{M}$ PBS, $30 \mathrm{cc} 0.1 \mathrm{M}$ citrate and $30 \mathrm{cc}$ demineralized water for 15 minutes. After rinsing with phosphate buffered saline (PBS), horse radish peroxidase (HRP) conjugated rabbit anti-FITC was applied in blocking buffer for 30 minutes. After three rinses in PBS, the peroxidase reaction was visualized with Novared (Vector Lab Inc., Burlingame; CA 940100).

Antibodies Rabbit anti-caspase-3 (Idun Pharmaceuticals, Inc., La Jolla, CA, USA), rabbit anti-MnSOD (a gift from dr.H.Verspaget, Dept. of Gastroenterology, LUMC, The Netherlands), rabbit anti Cu/Zn - SOD and rabbit anti Extra - Cellular (EC-) SOD (a gift from Dr. S. Marklund, Umea, Sweden), rabbit anti-vimentin (Euro Diagnostica, Arnhem, The Netherlands), mouse monoclonal anti-Ki 67 (DAKO, Glostrup, Denmark), mouse monoclonal anti cubulin (clone 2A3)(19)) and rabbit/mouse anti - AE1/AE3 (Neomarkers, LabVision, Fremont, CA, USA) were obtained as indicated.

Antigen retrieval Deparaffinized sections were first inactivated in $1,2 \% \mathrm{H}_{2} \mathrm{O}_{2}$ in methanol. In order to retrieve the antigen in the renal biopsy, sections were either boiled in $0.1 \mathrm{M}$ citrate $(\mathrm{pH} 6)$ for 1 minute or in $0.01 \mathrm{M}$ citrate $(\mathrm{pH} 6)$ for 10 minutes.

Double staining Specific antibodies directed against the brush border antigen cubulin of the proximal tubules and against the cytokeratin AE1/AE3 were used to achieve a sharper distinction between proximal and distal tubules. When anti-cubulin was used to localize proximal tubules it was applied first and followed by antigen retrieval with $0.1 \mathrm{M}$ citrate and incubation with anti-caspase-3, anti- Mn-SOD or anti Ki 67. Double staining for vimentin did not require antigen retrieval. When anti AE1/AE3 antibodies were used to localize 
distal tubules, kidney sections were incubated overnight with anti-vimentin followed by antigen retrieval for 10 minutes with $0.01 \mathrm{M}$ citrate and staining for AE1/AE3. When sections were stained for AE1/AE3 and Mn-SOD, antigen retrieval was performed first in $0.01 \mathrm{M}$ citrate followed by incubation with AE1/AE3 and subsequently with anti-Mn-SOD. When antibody binding was visualized by peroxidation of 3.3 diaminobenzidine tetrahydrochloride (DAB), either mouse or rabbit envision (Dako, Glostrup, Denmark) was used as a secondary antibody conjugated to HRP.When tissue binding was visualized using Fast Red (Klinipath, Duiven; The Netherlands) anti-mouse or anti-rabbit IgG antibodies were used conjugated to alkaline phosphatase (AF, Sigma, Zwijndrecht, The Netherlands). Finally double stained sections were counterstained for 1-2 minutes in hematoxylin.

\section{Immunohistochemical scoring}

The intensity of staining in tubular cells was scored semi quantitatively using a 3-point scale; 0: negative or weak intensity; $1+$ : intermediate intensity; 2+: strong intensity. The extent of staining was similarly scored as negative: $0-10 \%$ of tubules; focal: $10-50 \%$ of tubules or diffuse: $>50 \%$ of tubules. This scoring system was performed on proximal as well as on distal tubules, which were recognized on their immuno-histochemical or morphological characteristics. Scoring was performed at a magnification of $200 \mathrm{x}$. At time of scoring the investigators (LEs \& HeBo) were blinded for clinical and histological data. When immuno-histochemical markers to identify proximal or distal tubules were absent, their morphological characteristics were taken into account. Proximal tubular epithelial cells in ATN kidneys show a flat cytoplasm with few nuclei, giving them the impression of distal tubules or "distalisation". However, distal tubules have a high nucleus/cytoplasm ratio and their nuclei are better preserved in ATN than the proximal tubular cells, giving them a bead like appearance.

\section{Statistics}

The intensity and extent scores of the immunohistochemical stainings were compared between biopsies with and without histological ATN. The groups were compared with regard to the ordinal semiquantative scale of the scoring system of staining intensity and - extent, using non-parametric tests (Mantel-Haenzel chi-square test for linear association). The Kendall's tau-b coefficient shows the direction of this correlation. A p-value of 0.05 or less was considered significant. Statistical analysis was done using the SPSS software package (Version 10.0; SPSS, Inc., Chicago, IL).

\section{RESULTS}

Clinical characteristics of patients with or without delayed graft function (DGF) and with or without acute tubular necrosis (ATN)

A total of 41 biopsies was studied. Ten were taken from patients with DGF (Table 1) The remaining 31 were taken from patients with primary function (PF). Donor age for patients with DGF was significantly higher $(p<0.05)$ than for patients without DGF (Table 1$)$. Re- 
cipient age and cold ischemia time (CIT) did not differ between the 2 groups. ATN was observed in 16 biopsies (table 2), 5 (50\%) coincided with DGF, but 11 did not. The interval between transplantation and the timing of the biopsy did not differ between the two groups. Patients who experienced DGF but were not biopsied were slightly younger, had a younger donor and had lower Panel reactive antibodies (PRA). Other clinical characteristics summarized in table 2 , did not differ between the ATN and non-ATN group.

Table 1: Clinical characteristics of recipients with primary function (PF) or delayed graft function (DGF)

\begin{tabular}{l|ll} 
& PF & DGF \\
\hline Number of patients & 31 & 10 \\
Mean recipient age (yrs.) & $45(12)$ & $53(12)$ \\
Mean donor age (yrs.) & $35(14)$ & $48(11)^{*}$ \\
Mean Cold Ischemia Time (hrs.) & $23(12)$ & $28(11)$ \\
Biopsy interval (days) & $4.35(2.2)$ & $3.2(2.3)$ \\
ATN (\%) & $11(36)$ & $5(50)$ \\
PRA (\%) & $29(28)$ & $57(43)$
\end{tabular}

Chi-square test ${ }^{*} \mathrm{p}<0.05 ;$ (SD)

Table 2: Clinical characteristics of recipients with or without histological evidence ATN

\begin{tabular}{l|ll} 
& Non-ATN & ATN \\
\hline Number of biopsies & 25 & 16 \\
Mean recipient age (yrs.) & $45(11)$ & $50(15)$ \\
Mean donor age (yrs.) & $36(13)$ & $40(16)$ \\
Mean Cold ischemia time (hrs.) & $22(13)$ & $26(11)$ \\
Biopsy interval (days) & $3.9(2.1)$ & $4.4(2.4)$ \\
DGF (\%) & $5(20)$ & $5(31)$ \\
PRA (\%) & $38(35)$ & $31(35)$
\end{tabular}

\section{Histological parameters}

The morphological features of tubular necrosis in the biopsies with or without DGF are shown in Table 3. No correlation was found between the occurrence of DGF and the histological changes. Evaluation of the biopsies according to the Banff 1997 classification for transplant pathology (18) revealed no differences between the ATN and non-ATN group for the acute tubulitis ( $t$ )-score, mononuclear cell interstitial inflammation (i)-score and allograft glomerulitis (g)-score. All biopsies except for 2 biopsies in the non-ATN group and one in the ATN group, had grade $0 \mathrm{t}$-score. Six biopsies in the ATN group and 5 in the nonATN group showed a grade 1 interstitial inflammation (i) - score. No biopsy had a positive intimal arteritis $(\mathrm{v})$ - score. No histological differences between needle biopsies and wedge biopsies were present. 
Table 3: Histological changes observed in renal biopsies classified as Primary Function (PF) or. Delayed Graft Function (DGF)

\begin{tabular}{|c|c|c|c|}
\hline & $\operatorname{PF}(n=31)$ & $\operatorname{DGF}(n=10)$ & $\mathrm{p}$ \\
\hline \multicolumn{4}{|l|}{ Glomeruli } \\
\hline Mean number (SD) & $18.1(8.0)$ & $20.4(6.6)$ & \\
\hline Glomerulosclerosis (SD) & $0.61(0.89)$ & $0.59(0.71)$ & \\
\hline \multicolumn{4}{|l|}{ Tubules } \\
\hline \multicolumn{4}{|l|}{ Tubular necrosis } \\
\hline Present & 7 & 4 & 0.22 \\
\hline Absent & 24 & 6 & \\
\hline \multicolumn{4}{|l|}{ Cross sectional necrosis } \\
\hline Yes & 3 & 3 & 0.14 \\
\hline No & 28 & 7 & \\
\hline \multicolumn{4}{|l|}{ Tubular Shedding } \\
\hline Present & 12 & 7 & 0.21 \\
\hline Absent & 19 & 3 & \\
\hline \multicolumn{4}{|c|}{ Non-replacement phenomenon } \\
\hline Present & 8 & 3 & 0.86 \\
\hline Absent & 23 & 7 & \\
\hline \multicolumn{4}{|l|}{ Activated tubular cells } \\
\hline Present & 15 & 8 & 0.055 \\
\hline Absent & 16 & 2 & \\
\hline \multicolumn{4}{|l|}{ Mitosis } \\
\hline Present & 13 & 5 & 0.32 \\
\hline Absent & 18 & 5 & \\
\hline
\end{tabular}

\section{Markers for proximal and distal tubules}

In eleven out of 16 biopsies of the ATN group and 18 out of 25 biopsies of the non-ATN group, the cubulin positive brush border was absent, indicating damage to the brush border also in renal allografts without ATN. The presence or absence of cubulin in the brush border of proximal tubules did not differentiate between the presence or absence of ATN or DGF. The same applied to AE-1/AE-3 positivity and ATN or DGF: the AE-1/AE-3 marker for distal tubules was not only present in 23 out of 26 non-ATN biopsies, but also in 14 out of 16 ATN biopsies. However, when AE-1/AE-3 positivity was related to histological scores for ATN, it was correlated with the absence of necrosis or cross sectional necrosis. Twenty eight of the 37 biopsies with a positive staining for $\mathrm{AE}-1 / \mathrm{AE}-3$, had no signs of necrosis, whereas 2 biopsies with a negative staining for $A E-1 / A E-3$ had signs of necrosis (P: $0.02 ;:-0.23$ ). Thirty three of the 37 biopsies with a positive staining for $A E-1 / A E-3$, had no signs of cross sectional necrosis, whereas 2 biopsies with a negative staining for $A E-1 / A E-3$ had cross sectional necrosis ( $P: 0.03 ; \kappa:-0.33$ ) 
Active Caspase-3 and TUNEL staining

Of the sixteen biopsies classified as ATN, 5 showed no necrosis in the individual score. In these cases, the diagnosis of ATN was made on other diagnostic criteria: 3 showed "non replacement", 3 showed shedding of tubular epithelial cells in the lumen and in 3 biopsies activated tubular epithelial cells and mitosis were seen. The absence of necrosis prompted us to test markers for apoptosis.

Table 4 a shows that ATN was significantly associated with intensive staining for active caspase- 3 in the distal tubules $(p=0.04)$. The negative predictive value of a negative caspase-3 staining was calculated to be $78 \%$. On the other hand the positive predictive value was only 54\%. Five biopsies had ATN and DGF, whereas 21 biopsies were categorized as nonATN without DGF (non-ATN/non-DGF). When these categories were analyzed separately, the active caspase- 3 staining is positive in the distal compartment of all 5 biopsies from the ATN/DGF group, whereas 13 out of 20 biopsies in the non-ATN/ non DGF group are negative $(p=0.010)$ (Table $4 b)$. No statistical significant difference in the extent of the caspase-3 staining in these biopsies was found.

Interestingly, in the absence of ATN and subcomponents of ATN like tubular necrosis and cross-sectional necrosis, the distal tubules showed more often apoptosis as assessed by the staining with the TUNEL technique than in the presence of ATN $(p=0.01)$. The presence of TUNEL staining in the distal tubules predicted the absence of ATN in $96 \%$ of cases, whereas the absence of TUNEL predicted the presence of ATN in $60 \%$ of the cases. Furthermore tubular atrophy was positively correlated with the extent of TUNEL positivity (data not shown). In the proximal tubules TUNEL staining correlated with the intensity of the active caspase-3 staining $(p=0.04 ; \kappa: 0.23)$ (Table 5$)$. This was not the case in the other compartments.
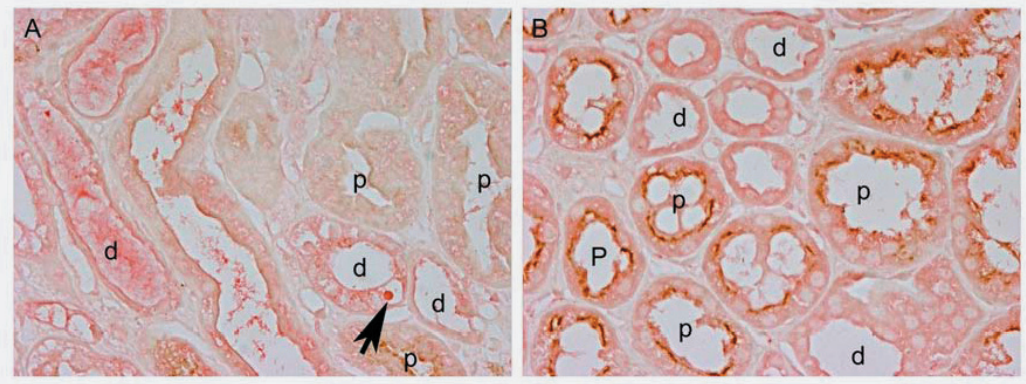

Figure 1

Active caspase-3 staining differentiating between the ATN (a) and the non-ATN group (b), in the distal tubules: Figure a shows the more prominent presence of active caspase-3 positive cells in the ATN group. The arrow points to a caspase-3 positive cell with histological apoptotic characteristics. $(\rightarrow)$ Original magnification: $\mathbf{4 0 0 x}$; proximal tubules: $\mathbf{p}$; Distal tubules: $\mathbf{d}$ 
Table 4a: Expression of active Caspase-3 in distal tubules in relation to ATN

\begin{tabular}{cllll} 
& ATN & & $\mathrm{p}$ & $\mathrm{K}$ \\
& Absent & Present & \\
\hline Active Caspase-3 (intensity) & & & & \\
Negative & 14 & 4 & & \\
+ & 4 & 4 & 0.04 & 0.31 \\
++ & 6 & 8 & & \\
\hline
\end{tabular}

${ }^{*} \mathrm{p}$ - value of the Mantel-Haenzel $\chi 2$ test for linear association ; ${ }^{* *}$ Kendall's tau $\beta$-correlation coefficient

Table 4b: Expression of active Caspase-3 in distal tubules in relation to ATN in 21 biopsies classified as histological non-ATN and clinical non-DGF versus 5 biopsies classified as histological ATN and clinical DGF

\begin{tabular}{cllll} 
& ATN & $\mathrm{p}$ & $\mathrm{K}$ \\
& Absent & Present & \\
\hline Active Caspase-3 (intensity) & & & \\
Negative & 13 & 0 & \\
+ & 3 & 1 & 0.010 & 0.49 \\
++ & 5 & 4 & & \\
\hline
\end{tabular}

${ }^{*} \mathrm{p}$ - value of the Mantel-Haenzel $\chi 2$ test for linear association ; ${ }^{* *}$ Kendall's tau $\beta$ - correlation coefficient

Table 5: Correlation between active caspase 3 staining and TUNEL staining in proximal tubules.

\begin{tabular}{cllll} 
& TUNEL & P & $\kappa$ \\
\hline Active Caspase-3 (intensity ) & $<10$ & $>10$ & & \\
Negative & 29 & 1 & & \\
+ & 5 & 0 & 0.04 & 0.23 \\
++ & 1 & 1 & & \\
\hline
\end{tabular}

${ }^{*} \mathrm{p}$ - value of the Mantel-Haenzel $\chi 2$ test for linear association ; ${ }^{* *}$ Kendall's tau $\beta$-correlation coefficient

\section{ROS deactivating enzymes}

As expressions of a protective response against ischemia, tubular localization of $\mathrm{Cu} / \mathrm{Zn}$ SOD, Ec-SOD and Mn-SOD were compared between the DGF and ATN groups. Cu/Zn-SOD localization was found in both proximal and distal tubules and the intensity and extent of staining did not differ between the groups. Ec-SOD localization was found in both proximal and distal tubules, but it was slightly more present in the distal tubules. However, there was no difference in intensity and extent of localization between the two groups (data not shown).

The intensity and extent of the Mn-SOD staining in distal tubules were related with the absence of DGF suggesting a protective role of Mn-SOD against the development of DGF (Table 6). This relationship was also found in the proximal tubules, but this relation was not significant. The predictive value for the absence of DGF, of distally located Mn-SOD was calculated to be $76 \%$, whereas the predictive value of absent Mn-SOD was not associated with the presence of ATN. 
Figure 2
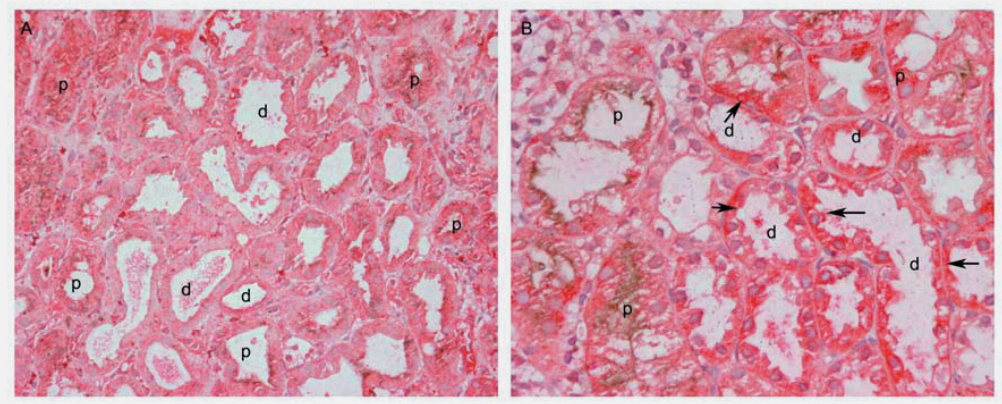

Distal tubular localization of Mn SOD in the presence (a) and absence (b) of DGF. Note the increased staining intensity in biopsies with DGF $(\rightarrow)$.

Original magnification: 400x; Proximal tubules: $\mathbf{p}$; Distal tubules: $\mathbf{d}$

Table 6: Relation presence of $\mathrm{Mn}$ SOD in distal tubules in relation to the occurrence of DGF.

\begin{tabular}{cllll} 
& $\begin{array}{l}\text { DGF } \\
\text { Absent }\end{array}$ & Present & $\mathrm{K}$ \\
\hline Mn SOD (Intensity) & & & \\
Negative & 5 & 3 & & \\
+ & 11 & 6 & 0.05 & -0.30 \\
++ & 15 & 1 & & \\
Mn SOD (Extent) & & & & \\
Negative & 5 & 3 & 0.06 & -0.31 \\
Focaal & 4 & 4 & & \\
Diffuse & 22 & 3 & & \\
\hline
\end{tabular}

${ }^{*} \mathrm{p}$ - value of the Mantel-Haenzel $\chi 2$ test for linear association ; ${ }^{* *}$ Kendall's tau $\beta$ - correlation coefficient

\section{Vimentin staining}

Regeneration was assessed by staining for vimentin. Vimentin localization was seen relatively more often in ATN biopsies than in the non-ATN biopsies. The basolateral localization of vimentin was exclusively seen in cubulin positive proximal tubules. However, the difference with non ATN biopsies was not significant ( $p=0.07$ ).

\section{Ki 67 staining}

The proliferation marker Ki 67 was not associated with the occurrence of ATN or DGF. However the extent of the activated Mn-SOD staining in the proximal tubules was associated with a higher extent of staining for Ki 67, but this association was not significant ( $p$ 0.09; data not shown). No significant correlation was found between Ki-67 staining and the expression of vimentin. 
Figure 3

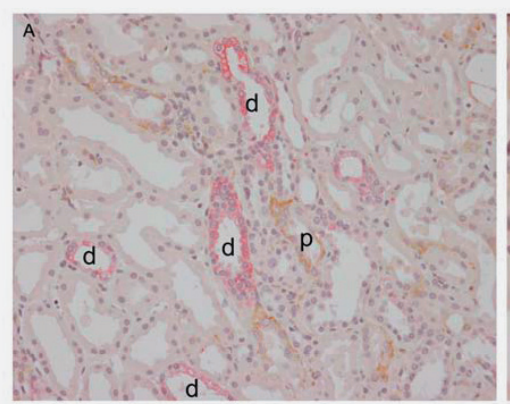

Magnification $200 x$

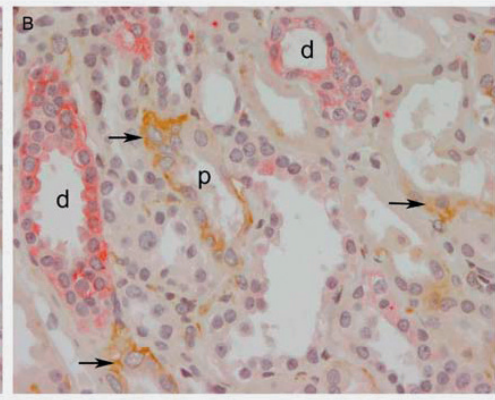

Magnification $400 \mathrm{x}$

Basolateral vimentin staining in proximal tubules $(\rightarrow)$, which is present relatively more frequent in the presence of ATN. Note the increased staining intensity in biopsies with ATN.

Original magnification: A :200x; B : 400x. Proximal tubules: $\mathbf{p}$; Distal tubules: d

\section{DISCUSSION}

This study confirms earlier reports documenting the poor correlation between the defined clinical syndrome of delayed graft function (DGF) and the morphological changes of ATN in renal allografts $(2,20)$. The ATN score in this study was performed according to the criteria of Olsen and Solez (16) and did not correlate with the presence of DGF (Table 1). When a functional definition of DGF was used, excluding acute rejection episodes and cyclosporin as a cause of DGF, the presence of ATN in renal allografts predicted DGF, in only $50 \%$ of the cases, whereas the absence of ATN predicted the absence of DGF in $80 \%$ of our cases (Table 2). This prompted us to study molecular markers allegedly involved in the pathogenesis of DGF hoping to obtain a more reliable histological marker for the presence of DGF and to gain more insight in the pathophysiology of DGF. The markers were selected on the basis of the generally accepted pathogenetic events in ATN, consisting of an initiation phase of ischemic and reperfusion injury, an extension or a maintenance phase in which the renal tubular protective response is important and a recovery phase in which tubular regeneration and proliferation are characteristic (17).

Necrotic tubular cells have been described to undergo the following morphological changes: first proximal tubular cells form "blebs" in their apical membranes and lose their brush border (21). They lose their polarity and the integrity of their tight junctions is disrupted, presumably as a consequence of the redistribution of the Na/K-ATPases, resulting in alterations in the cytoskeletal network and disruption of tubular cells. Dead tubular cells slough into the tubular lumina and therefore are responsible for the denuded aspect of the lumen (non-replacement phenomenon) and the tubular obstruction (20). The denuded tubular basement membrane (TBM) is repopulated by proliferation of adjacent viable cells, under the influence of growth factors and by dedifferentiated stem cells that first spread out over the membrane, giving the proximal tubules the aspect of distal tubules (distalisation of proximal tubules). In contrast to normal distal tubules these recovering proximal tubules show very few nuclei. After the TBM has been repopulated cells will proliferate and dedif- 
ferentiate until normal morphology and function is reinstalled.

Since the morphological identification of damaged renal tubular segments during ATN is difficult, we tried to improve the recognition of these segments by introducing proximal and distal specific markers (cubulin and AE- $1 / \mathrm{AE}-3$ respectively). These markers enabled us to identify the distal tubules in the presence as well as in the absence of ATN. The presence of $\mathrm{AE}-1 / \mathrm{AE}-3$ positivity, allowed us to identify proximal tubules, as tubules that were $\mathrm{AE}-1 /$ $A E-3$ negative in $A E-1 / A E-3$ positive biopsies. In the four AE-1/AE-3 negative biopsies, distal tubules were identified on morphological grounds.

The paradox of severe reduction of glomerular filtration rate (GFR) and mild tubular necrosis could be explained by cell death via apoptosis instead of necrosis (22). The contribution of apoptosis was evaluated using the TUNEL technique and an immuno-histochemical staining for active caspase-3. In our hands the TUNEL technique had to be modified to obtain a higher specificity at the expense of sensitivity, because of the high percentage of false positive results in biopsies of normal kidneys. TUNEL positivity in the proximal tubules of both groups did not differ. Proximal tubules were by regular light microscopy either vital or necrotic. In the latter case intra-luminal sludging of cubulin positive material was present. Necrosis of proximal tubular cells is probably based on the high sensitivity of the proximal tubules for oxygen deprivation and ATP-depletion (23). Staining for active caspase-3 on the other hand was more prominent in the distal tubules in biopsies with ATN. The presence of apoptosis instead of necrosis in the distal tubules of the ATN group can be explained by a lower susceptibility of the distal tubules to ischemia and reperfusion injury followed by subsequent necrosis. Only when ATP depletion becomes more severe distal tubules will react with necrosis instead of apoptosis (24). Our finding that in selected cases in which extremes of ATN and DGF and non-ATN and non-DGF are compared, the relation of DGF with the extent and intensity of active caspase- 3 staining in distal tubules is even stronger, supports this hypothesis. Experimental studies illustrate that various zones within the kidney, are differently susceptible to ischemic damage (25). Since the amount of tissue in human biopsies is very limited in a retrospective study, identification of these separate segments within the nephron was not attempted.

Activated caspases affect the cytoskeletal filaments, including actin. Recent studies have shown that the disruption of cytoskeletal proteins may in itself induce apoptosis and cellular detachment (9). ATN is characterized ultrastructurally by disruption of apical and basolateral membranes of proximal tubules, with redistribution and diminished intensity of cytoskeletal proteins of the apicolateral membrane (21). These mechanisms might contribute to the pathogenesis of ATN. Because of the resemblance between these features of ATN and the mechanisms of action of activated caspases, apoptosis might be the "missing link" in the pathogenesis of ATN. Activation of caspase-3 has been described to be the final step in the apoptotic pathway (27). When biopsies were stained for active caspase-3, cytoplasm of distal tubules stained significantly more intense in the ATN group (Table 4a). This made us conclude that the absence of distal active caspase-3 staining predicted the absence of ATN in $78 \%$ of cases, whereas the presence of caspase- 3 predicted ATN in $75 \%$ of cases. No differences were found in the proximal tubules between the two groups.

TUNEL positivity was more prevalent in the distal tubules, especially in the non-ATN group. This unexpected finding could be explained by experimental evidence that apoptosis is not only an expression of damage, but also of repair $(28,29)$. The number of apoptotic cells 
in rat kidneys with experimental ATN were found to peak in the early phase after ischemic injury and in a later stage during regeneration. The first phase of apoptosis occurs early on, between 12 and 48 hours after the acute ischemic or nephrotoxic insult and is a result of the damaging effect of the insult. In contrast, the apoptosis associated with the recovery phase has been postulated to contribute to the remodeling of injured tubules and to facilitate their return to a normal structural and functional state. The discrepancy between the TUNEL and active caspase-3 staining might be explained by the fact that the specificity for apoptosis of the TUNEL staining is not high enough. It is known from experimental models that after treatment with carbon tetrachloride $(\mathrm{CCl} 4)$ or N-nitrosomorpholine (NNM), rat livers showed a high percentage of TUNEL positive necrotic liver cells (26)

Since reactive oxygen species play an important role in the pathogenesis of ATN, the expression of enzymes with a protective effect against these oxygen radicals was studied. Superoxide dismutases have been found to be involved in the detoxification of reactive oxygen species (30) and have been described to play a role in experimental ischemia reperfusion injury (31). In humans at least three isoforms of SOD have been identified, each with a distinct metal component: Copper/Zinc-SOD (Cu/Zn-SOD), Manganese-SOD (MnSOD) and Extracellular-SOD (Ec-SOD). We did not find any difference for the Cu/Zn-SOD or Ec-SOD expression, between the two groups. Mn- SOD was found to be the only inducible form and to be exclusively located in the mitochondria (32). The overexpression of Mn-SOD was reported to correlate with the protection against pro-apoptotic stimuli and ischemic damage whereas decline of $\mathrm{Mn}-\mathrm{SOD}$ expression has been associated with disease activity, like cancer and transplant rejection and cis-platinum tubulotoxicity (33-35). In biopsies from the non-DGF group, we found a more extensive presence of Mn-SOD in the distal tubules, compared with biopsies from the DGF group. Mn-SOD expression was also present in the proximal tubules, but there was no difference between the groups. These findings suggest that distal tubules are more resistant against Reactive Oxygen Species because they are capable of generating protective upregulation of Mn-SOD. The negative predictive value of the presence of Mn-SOD in the distal tubules predicts the absence of DGF in $76 \%$ of cases. This could explain the absence of necrosis but the presence of apoptosis in distal tubules: Proximal tubular cells have been reported to be more vulnerable to necrosis (36) whereas distal tubules may be protected to some extent against necrosis because of their upregulation of Mn-SOD (14). The clinical use of recombinant SOD (rh-SOD), to prevent ischemia reperfusion injury and subsequent DGF was tested in two randomized trials $(37,38)$. Both studies showed that with the pharmacological doses that were used, no effect on the incidence of DGF was found. However one study showed a lower incidence of acute and chronic rejection and a better long-term graft function for patients treated with RhSOD (38). We therefore hypothesize that at the time of transplantation, the presence of $\mathrm{Mn}$ SOD, either structurally, upregulated or substituted, may be a parameter for the intrinsic potential to recover and may be a surrogate marker for long-term outcome of grafts.

After necrosis and/or apoptosis have occurred, tubular cells will regenerate (39). We found that the vimentin expression in proximal tubular cells was more prominent in biopsies, showing denudation, one of the morphological characteristics of ATN in grafts. However, this did not reach the level of significance. Tubular cells are of mesenchymal origin, but normal tubular cells do not express the mesenchymal intermediate filament protein vimentin. After being damaged, the recovery of the tubular cells is characterized by replace- 
ment of irreversibly injured tubular epithelial cells by surviving tubular cells, renal stem cells or cells originating from the bone marrow. These cells differentiate to tubular cells, meanwhile expressing antigens, mimicking renal organogenesis $(40,41)$. Vimentin expression has been described to be increased in damaged tubular cells and in renal carcinomas as a sign of tubular dedifferentiation $(42,43)$. However, we could not confirm their findings in our study, because of the lack of significance. The expression of vimentin that we observed in proximal tubules of the ATN group can be interpreted as a sign of regeneration. Unfortunately no correlation was found between the presence of the proliferation marker Ki 67 in tubular cells and the occurrence of ATN or DGF. However,Ki-67 positivity in proximal tubules was more extensive in biopsies with a high expression of the protective marker $\mathrm{Mn}-\mathrm{SOD}$. This means that also the presence of $\mathrm{Mn}-\mathrm{SOD}$ in proximal tubules correlated with its capacity for regeneration and recovery.

Since biopsies of human kidneys with ATN are difficult to obtain, we studied allograft biopsies taken to detect rejection during the first week after transplantation. Because acute rejection and calcineurine toxicity were excluded, only 41 biopsies were available for this study. This study shows that the use of molecular markers in the evaluation of early posttransplant renal biopsies does not discriminate between patients with DGF and/or ATN. However, in distal tubules correlations were found between the expression of active caspase- 3 and the presence of ATN. This could explain why half of our patients with DGF did not show signs of ATN in their biopsies. Furthermore the expression of Mn-SOD in distal tubules is related to a lower occurrence of DGF, suggesting a protective role. Since the absence of distal localization of active caspase- 3 is associated with the absence of ATN in $78 \%$ of patients with DGF and the absence of Mn-SOD in the distal tubules is associated with the absence of DGF, these markers should be evaluated in a prospective study with protocolized biopsies. 


\section{REFERENCES}

1. Rosen S, Heyman SN. Difficulties in understanding human "acute tubular necrosis": limited data and flawed animal models. Kidney Int 2001:60: 1220-1224.

2. Solez K, Lilliane Morel-Maroger, Jean-Daniel Sraer. The Morphology of "Acute Tubular Necrosis" in man: Analysis of 57 Renal Biopsies and a comparison with the glycerol model. Medicine 1979:58:362-376.

3. Boom H, Mallat MJ, De Fijter JW, Zwinderman AH, Paul LC. Delayed graft function influences renal function, but not survival. Kidney Int 2000:58: 859-866.

4. van Es LA, Sijpkens YW, Paul LC. Surrogate markers of chronic allograft nephropathy. Ann Transplant 2000:5: 7-11.

5. Olsen S, Burdick JF, Keown PA, Wallace AC, Racusen LC, Solez K. Primary acute renal failure ("acute tubular necrosis") in the transplanted kidney:morphology and pathogenesis. Medicine (Baltimore) 1989:68: 173-187.

6. Solez K, Racusen LC, Marcussen N et al. Morphology of ischemic acute renal failure, normal function, and cyclosporine toxicity in cyclosporine-treated renal allograft recipients. Kidney Int 1993:43: 10581067.

7. Takeda M, Shirato I, Kobayashi M, Endou H. Hydrogen peroxide induces necrosis, apoptosis, oncosis and apoptotic oncosis of mouse terminal proximal straight tubule cells. Nephron 1999:81:234-238.

8. Edelstein CL, Shi Y, Schrier RW. Role of caspases in hypoxia-induced necrosis of rat renal proximal tubules.J Am Soc Nephrol 1999:10: 1940-1949.

9. White SR, Williams P, Wojcik KR et al. Initiation of apoptosis by actin cytoskeletal derangement in human airway epithelial cells. Am J Respir Cell Mol Biol 2001:24: 282-294.

10. Brown SB, Bailey K, Savill J. Actin is cleaved during constitutive apoptosis. Biochem J 1997:323: 233237.

11. Chatterjee PK, Brown PA, Cuzzocrea S et al. Calpain inhibitor-1 reduces renal ischemia/reperfusion injury in the rat. Kidney Int 2001:59: 2073-2083.

12. Alejandro V, Scandling JD, Jr., Sibley RK et al. Mechanisms of filtration failure during postischemic injury of the human kidney. A study of the reperfused renal allograft. J Clin Invest 1995:95: 820-831.

13. Oury TD, Chang LY, Marklund SL, Day BJ, Crapo JD. Immunocytochemical localization of extracellular superoxide dismutase in human lung. Lab Invest 1994:70: 889-898.

14. Dobashi K, Ghosh B, Orak JK, Singh I, Singh AK. Kidney ischemia-reperfusion: modulation of antioxidant defenses. Mol Cell Biochem 2000:205: 1-11.

15. Holthofer $\mathrm{H}$, Miettinen A, Lehto VP, Lehtonen $\mathrm{E}$, Virtanen I. Expression of vimentin and cytokeratin types of intermediate filament proteins in developing and adult human kidneys. Lab Invest 1984:50: 552559.

16. Olsen S., Burdick J.F., Keown P.A., Wallace A.C., Racusen L.C., Solez K. Primary Acute Renal Failure („Acute Tubular Necrosis") in the transplanted Kidney: Morphology and Pathogenesis. Medicine 1989: 173187.

17. Sutton TA, Fisher CJ, Molitoris BA. Microvascular endothelial injury and dysfunction during ischemic acute renal failure. Kidney Int 2002:62: 1539-1549.

18. Racusen LC, Solez K, Colvin RB et al. The Banff 97 working classification of renal allograft pathology. Kidney Int 1999:55: 713-723.

19. Verroust PJ, Christensen El. Megalin and cubilin--the story of two multipurpose receptors unfolds. Nephrol Dial Transplant 2002:17: 1867-1871. 
20. Racusen L.C., Fivush B.A., Li Y.L., Slatnik I., Solez K. Dissociation of tubular cell detachement and tubular cell death in clnicial and experimental "acute tubular necrosis". Laboratory Investigation 1991:64: 546556.

21. Molitoris B.A. Ischemia-induced loss of epithelial polarity: potent role of the actin skeleton. Am J Physiol 1991:260:F769-F778.

22. Lieberthal W, Levine JS. Mechanisms of apoptosis and its potential role in renal tubular epithelial cell injury. Am J Physiol 1996:271:F477-F488.

23. Brezis M, Rosen S. Hypoxia of the renal medulla--its implications for disease. N Engl J Med 1995:332: 647-655.

24. Vaux DL, Korsmeyer SJ. Cell death in development. Cell 1999:96:245-254.

25. Brezis M, Rosen S, Silva P, Epstein FH. Selective vulnerability of the medullary thick ascending limb to anoxia in the isolated perfused rat kidney. J Clin Invest 1984:73: 182-190.

26. Grasl-Kraupp B, Ruttkay-Nedecky B, Koudelka H, Bukowska K, Bursch W, Schulte-Hermann R. In situ detection of fragmented DNA (TUNEL assay) fails to discriminate among apoptosis, necrosis, and autolytic cell death: a cautionary note. Hepatology 1995:21:1465-1468.

27. Thornberry NA, Lazebnik Y. Caspases: enemies within. science 1998:281:1312-1316.

28. Lieberthal W, Koh JS, Levine JS. Necrosis and apoptosis in acute renal failure. Semin Nephrol 1998:18: 505-518.

29. Zager RA, Fuerstenberg SM, Baehr PH, Myerson D, Torok-Storb B. An evaluation of antioxidant effects on recovery from postischemic acute renal failure. J Am Soc Nephrol 1994:4: 1588-1597.

30. Fridovich I. Superoxide radical and superoxide dismutases. Annu Rev Biochem 1995:64:97-112.: 97112.

31. Yin M, Wheeler MD, Connor HD et al. Cu/Zn-superoxide dismutase gene attenuates ischemia-reperfusion injury in the rat kidney. J Am Soc Nephrol 2001:12:2691-2700.

32. Zhao Y, Xue Y, Oberley TD et al. Overexpression of manganese superoxide dismutase suppresses tumor formation by modulation of activator protein-1 signaling in a multistage skin carcinogenesis model. Cancer Res 2001:61:6082-6088.

33. Davis CA, Nick HS, Agarwal A. Manganese superoxide dismutase attenuates Cisplatin-induced renal injury: importance of superoxide. J Am Soc Nephrol 2001:12: 2683-2690.

34. Janssen AM, Bosman CB, van Duijn $W$ et al. Superoxide dismutases in gastric and esophageal cancer and the prognostic impact in gastric cancer. Clin Cancer Res 2000:6:3183-3192.

35. Macmillan-Crow LA, Cruthirds DL. Invited review: manganese superoxide dismutase in disease. Free Radic Res 2001:34:325-336.

36. Shanley PF, Rosen MD, Brezis M, Silva P, Epstein FH, Rosen S. Topography of focal proximal tubular necrosis after ischemia with reflow in the rat kidney. Am J Pathol 1986:122:462-468.

37. Pollak R., Andrisevic JH, Maddux MS, Gruber SA, Paller MS. A randomized double-blind trial of the use of human recombinant superoxide dismutase in renal transplantation. Transplantation 1993:55:57-60.

38. Land W, Schneeberger H., Schleibner S. et al. The beneficial effect of human recombinant superoxide dismutase on acute and chronic rejection events in recipients of cadaveric renal transplants. Transplantation 1994:2:211-217.

39. Imgrund $\mathrm{M}$, Grone $\mathrm{E}$, Grone $\mathrm{HJ}$ et al. Re-expression of the developmental gene Pax-2 during experimental acute tubular necrosis in mice 1. Kidney Int 1999:56: 1423-1431.

40. Safirstein R. Gene expression in nephrotoxic and ischemic acute renal failure.J Am Soc Nephrol 1994:4: 1387-1395.

41. Kale S, Karihaloo A, Clark PR, Kashgarian M, Krause DS, Cantley LG. Bone marrow stem cells contribute 
to repair of the ischemically injured renal tubule.J Clin Invest 2003:112:42-49.

42. Ward JM, Stevens JL, Konishi N et al. Vimentin metaplasia in renal cortical tubules of preneoplastic, neoplastic, aging, and regenerative lesions of rats and humans. Am J Pathol 1992:141:955-964.

43. Grone HJ, Weber K, Grone E, Helmchen U, Osborn M. Coexpression of keratin and vimentin in damaged and regenerating tubular epithelia of the kidney. Am J Pathol 1987:129: 1-8. 


\section{DELAYED GRAFT FUNCTION IN RENAL TRANSPLANTATION}

Henk Boom, Leendert C. Paul, Johan W. de Fijter

Transplantation Reviews 2004 ;18; 3: 139-152 


\section{INTRODUCTION}

Delayed graft function (DGF) in renal transplantation is an enigmatic problem.

Progress in the research after the etiology and consequences of DGF are hampered because the clinical consequences of DGF on long term graft function are unclear and renal biopsies are usually not taken to document the cause of the DGF syndrome but rather to exclude additional acute rejection episodes. Our knowledge on DGF is mainly based on studies in experimental animals and on clinical data on acute renal failure in native kidneys. This comparison has major flaws, because the risk factors and clinical setting for acute renal failure in the transplantation setting are substantially different from the risk factors for acute renal failure in native kidneys.

The interest in DGF has increased with the increased use of marginal donors, including non-heart-beating donors, donors at the extremes of age, and donors with hypertension and diabetes, in order to resolve the shortage of kidney donors. This group of donors experience DGF more frequently, with an incidence of upto $50 \%$ (1-6).

The underlying mechanism is considered to be related to ischemic and reperfusion damage, which may be further complicated by an increased likelihood of acute rejection episodes $(3,7)$ or drug-related nephrotoxicity $(8)$.

There is debate on the impact of DGF on late graft outcome. Some authors reported an effect of DGF on renal allograft survival $(9,10,10)$, while others only found inferior graft survival in patients who also experienced acute rejection episodes $(11,12)$.

One possible explanation for this apparent difference in outcome may be the definition of DGF that is used. To study risk factors for DGF and its clinical consequences, it is therefore very important to use a definition of DGF, in which the contribution of ischemia and reperfusion injury is stressed.

\section{Definition of DGF}

In most studies DGF is defined as the need of dialysis treatment in the first week after renal transplantation. This is a criterion that is easy to register and to obtain from large databases (13). However, dialysis during the first week after transplantation is also performed for other reasons than DGF, like hyperkaliemia and / or fluid overload. Another flaw in this definition is the inability to exclude acute rejection and calcineurin toxicity as an additional cause of impaired graft function. For that reason others have defined DGF as a functional parameter distinct from the need of dialysis and used the time needed to achieve an arbitrarily defined creatinine clearance as a marker for delayed graft function (9),(3,14). Using this definition they found an impact of DGF on short term graft survival and function, when DGF lasted for at least 1 week and rejection episodes were excluded.

Because the pathogenesis of DGF is supposed to be of ischemic origin we propose to define DGF in clinical studies retrospectively on the basis of renal function, distinct from the need of dialysis treatment. Using the absence of a spontaneous decrease of serum creatinine of more than $10 \%$ per day for at least 3 consecutive days within 1 week after transplantation, excluding acute rejection, and calcineurin inhibitor toxicity as a possible cause of this DGF, allows a more meaningful analysis of the risk factors and consequences of 
ischemic damage and associated reperfusion injury that is supposed to be the underlying cause of DGF.

\section{Acute ischemic renal injury and recovery}

In the pathogenesis of acute ischemic failure 3 stages can be recognized (15). The first stage is the ischemic phase in which ischemic and reperfusion injury takes place and in which renal epithelial and endothelial cells are subjected to lethal insults leading to apoptosis and /or necrosis (16). The maintenance phase represents a phase of stabilization of injury by intrinsic or upregulated defense mechanisms. During this phase, events leading to cellular repair, proliferation and redifferentiation. lead to the recovery phase in which epithelial en endothelial function improve, leading to the recovery of renal function.

\section{Ischemic phase of ATN}

Ischemia and reperfusion injury in acute tubular necrosis (ATN)

During the ischemic phase, renal metabolism is characterized by severe ATP depletion due to a lack of oxygen and a subsequent shift from aerobic to anaerobic metabolism (Fig 1.) (17). This leads to a disruption of cellular homeostasis, resulting in failure of the cellular sodium-potassium pumps as well as the calcium pumps (18). (19). Because of an increase in cytosolic calcium levels calcium dependent enzymes like cystein proteases, phospholipases and endothelial nitric oxide synthetase (e-NOS) will be activated (20). These enzymes are able to break-down the cytoskeleton of the cells, eventually leading to cell death. Next to this purely ischemic condition, hypoxanthine is formed from xanthine which are degradation products of adenosine triphosphate (ATP) (21).

After the reinstitution of perfusion several factors contribute to the further damage. First of all the sudden increase of perfusion pressure causes endothelial damage and inflammation. Next molecular oxygen $\left(\mathrm{O}_{2}\right)$ is reintroduced, during reperfusion, which reacts with hypoxanthine which forms Reactive Oxygen Species (ROS). The free radical members of $\mathrm{ROS}$ are Superoxide $\left({ }^{\circ} \mathrm{O}_{2}\right)$ and the hydroxyl radical $\left({ }^{\circ} \mathrm{OH}\right)$ and are probably the most important biologically active free radicals, leading to extensive damage of the cell membranes by affecting its integrity. These processes eventually result in cellular apoptosis and / or cell death (22-24),(25). The cellular characteristics of this ischemic phase consist mainly of apoptosis and necrosis.

Apoptosis or programmed cell death is an active, energy dependent process with morphological characteristics that differ markedly from necrosis. The essence of apoptosis is a process of cellular auto-digestion, which is regulated by activation and inhibition of enzymes and which are identical for all human tissues. The key molecules are proteases named caspases of which the caspase- 3 is the enzyme that is the end of the final common path way (Fig.2). Upon activation of caspase-3, DNA is fragmented leading to the characteristic apoptotic bodies. Because of subsequent changes in the plasma membranes, the cells are removed by phagocytic cells.

Necrosis is seen in experimental animal models but only in a minority of the human cases (26-28). It is usually patchy involving individual cells or small clusters of cells sometimes re- 
Figure 1. Schematic representation of the apoptotic cascade Ischemia

Reperfusion

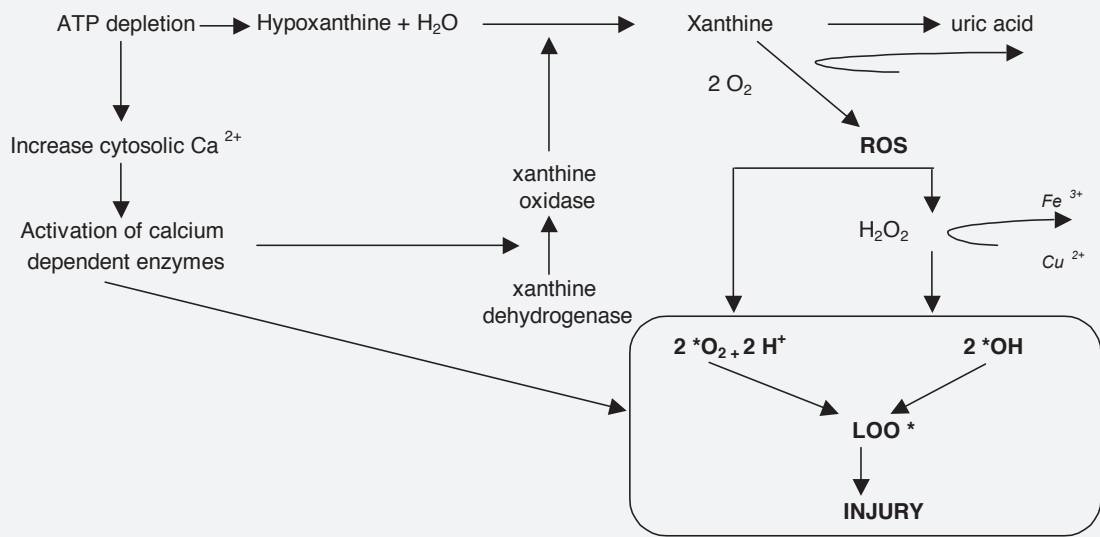

sulting in small areas of denuded basement membrane (non-replacement phenomenon). Tubular cell necrosis is associated with a rapid metabolic collapse, cell swelling and early loss of plasma membrane integrity and the loss of the polarity and the integrity of their tight junctions is disrupted, perhaps as a consequence of alterations in the cytoskeletal network $(18,29)$. Because of the redistribution of the $\mathrm{Na} / \mathrm{K}$-ATPases, tubular function is disturbed and cells die. This in turn leads to the release of proteolytic enzymes and other injurious cytosolic components into the extracellular space that incites an inflammatory response. More subtle changes include loss of brush border, flattening of the epithelium, detachment of cells, intratubular cast formation and dilation of the tubules (26-28) (Fig.3).

Figure 2. Schematic representation of the ischemia and reperfusion injury cascade
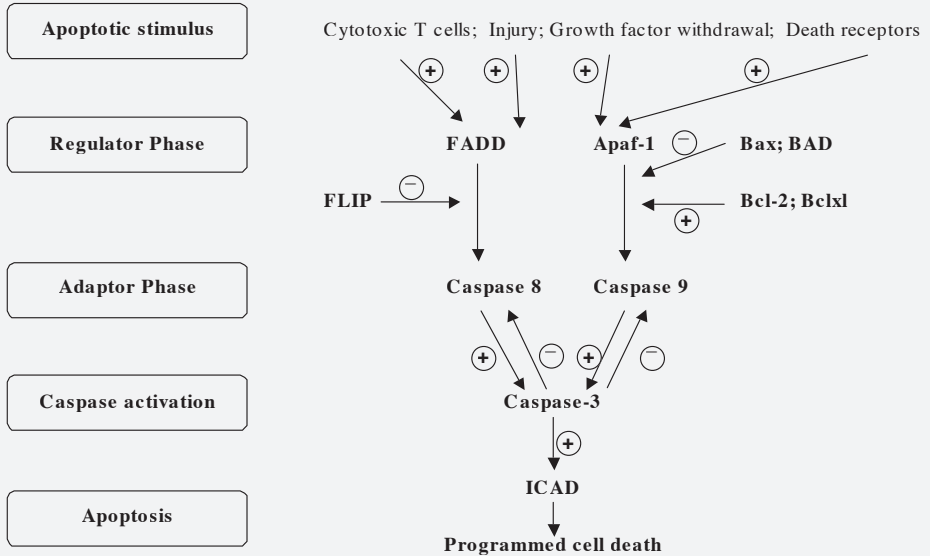
Figure 3. Cell biological characteristics of the acute tubular necrosis

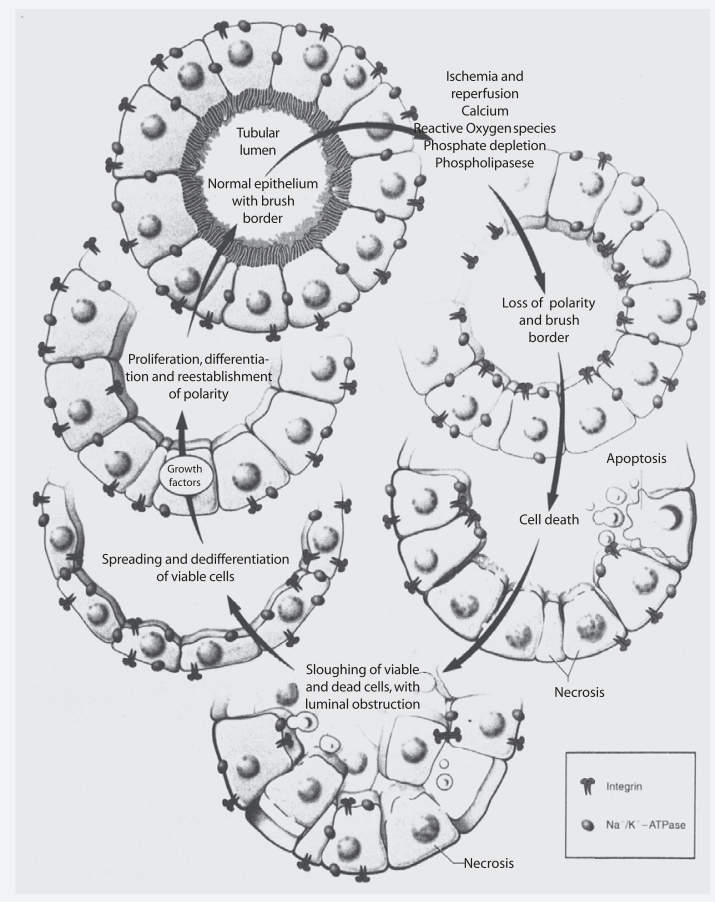

Reprinted with permission (98)

\section{Maintenance phase of ATN}

The kidney has naturally present anti-oxidant enzymes to counteract the effects of the free radicals. The catalases and gluthathion peroxidase act by safely decomposing the peroxides. The superoxide dismutases (SOD) act by scavenging the free radicals especially the ${ }^{\circ} \mathrm{O}_{2}$. In humans it is present in at least two forms, the cytoplasmatic copper/zinc (CuZn)-SOD and the mitochondrial manganese (Mn)-SOD.SODs are enzymes that catalyze the dismutation of ${ }^{\circ} \mathrm{O}_{2}$ to hydrogen peroxide $\left(\mathrm{H}_{2} \mathrm{O}_{2}\right)$, which is decomposed, by other enzymes like catalase and gluthation peroxidase (22). The presence and the (down regulated) activity of these SOD's seem to be related to the amount of damage induced by ROS in rat kidneys $(30,31)$. However, the clinical use of human recombinant superoxide dismutase, did not protect against DGF in human kidneys $(32,33)$. During the maintenance phase tubular cells share characteristics of the ischemic phase in which necrosis, apoptosis and the interstitial infiltrate are present and characteristics of the recovery and regeneration phase in which proliferation and redifferentiation are present. 


\section{Recovery phase of ATN}

This process is regulated by the expression of a number of transcription factors, structural proteins and growth factors and is a copy of kidney organo-genesis in respect to the high rate of DNA synthesis, like PCNA, the expression of apoptosis and the expression of genes that encode for processes during renal organogenesis like keratin and vimentin (34) $(35,36)$. On ischemia and reperfusion injury growth factors like the hepatic growth factor, insulin like growth factors and fibroblast growth factors are upregulated $(37,38)$. Others such as epidermal growth factors are down regulated in injured proximal tubules (39). Treatment with these growth factors in animal models was promising $(37,40,41)$ but the use in humans is still limited (42). During recovery from ischemia and reperfusion injury, surviving tubular epithelial or renal stem cells, differentiate and proliferate, eventually replacing the irreversibly injured tubular epithelial cells and restoring tubular integrity $(43,44)$. Morphologically this is characterized by the presence of mitotic figures and signs of cell proliferation. This process enables the replacement of the damaged epithelium and is maximal at 24 to 48 hours after ischemic injury in the rat. (45).

\section{Risk factors of DGF}

Using the earlier-mentioned functional definition of DGF, risk factors for post transplant acute renal failure (DGF) can be divided in donor-related factors, transplantation-related factors and recipient related risk factors. The cadaveric kidney is theoretically subjected to the cumulative damage at every step along the way from procurement to reperfusion whereas kidneys from living donors rarely develop DGF.

\section{Risk factors related to the donor}

Well known donor related risk factors for DGF are donors of over 50 years of age and an elevated serum creatinine or suboptimal renal function of the donor. In human adults, renal blood flow (RBF) declines by approximately $10 \%$ per decade after the age of 40 . This reduction of RBF is partially explained by a reduction of renal mass but is accompanied by an increased afferent and efferent arteriolar resistance especially in the renal cortex $(46,47)$. This change in glomerular filtration rate (GFR) is hard to detect when serum creatinine alone is taken into account: Muscle mass and consequently urinary creatinine excretion, decreases with age. This implies that for the same serum creatinine concentration, GFR in the elderly can be severely impaired in comparison to younger adults. Kidneys from older individuals have several structural and functional changes compared with kidneys from younger donors. Longitudinal studies of elderly individuals have shown a diminution in renal reserve, along with functional constraints on the kidney's ability to respond appropriately to challenges of either excesses or deficits (48). Studies of kidneys obtained at autopsies demonstrated a progressive decrease in the number and size of glomeruli with age, resulting in a progressive decrease of the glomerular filtration volume $(49,50)$. In addition to the loss of glomeruli, there is an age-dependent increase in the cortical interstitial volume as a result of progressive interstitial fibrosis $(49,51)$. Most renal biopsies from kidney donors who are older than $40 \mathrm{yr}$ show intimal fibrosis in the smaller arteries, arteriolar hyalinosis, and inter- 
stitial fibrosis (52). Not only factors intrinsically related to the donor, but also events preceding brain death and harvest of the kidney contribute to the occurrence of DGF. Before the establishment of brain death of the potential donor, the kidney may be damaged by the underlying disease process (e.g. hypotension or shock), or by the therapeutic maneuvers instituted in an attempt to revive the patient or to maintain circulation after brain death, like the use of dopaminergic medication and resuscitation procedures (53). Decreasing platelet count and disseminated intravascular coagulation are frequently found and at least suggest that endothelial injury or dysfunction may already be present before the organs are harvested. During episodes of cardiac arrest or prolonged hypotension, the kidney will suffer from warm ischemia and reperfusion injury. Catecholamine release and pharmacological inotrope agents may contribute to intrarenal vasospasms leading to areas in the kidney subjected to relative hypoperfusion. Because the donor generally is in a catabolic state, recovery from ischemic damage is more difficult. After brain death but before explantation, the potential donor may not be considered a high priority for surgery in the setting of a busy intensive care unit and resuscitation may be delegated to those with limited experience in appropriate care (54).

\section{Risk factors related to the transplantation procedure}

Organ procurement also contributes to the development of DGF. This starts with hypoperfusion after circulation of the donor has stopped (warm ischemia time [WIT]). However, with the multi organ procurement procedures currently this WIT is almost reduced to zero. However, with the raising interest of non-heart beating procedures, WIT has nowadays become a serious contributor to DGF (55), (56). During surgery errors in line placement can result in inadequate flushing of blood and / or cooling, and undue manipulation of renal arteries can induce vascular spasm (53). The most important independent and robust risk factor is the time that it takes from the explantation of the kidney until its transplantation into the recipient, defined by the cold ischemia time (CIT) (57-63). The type of preservation fluid also is recognized as a risk factor for DGF in a study in the Eurotransplant area, in which the preservation fluid developed by the University of Wisconsin (UW) appeared to be superior to Euro Collins (EC) (64). After perfusion is reestablished several mechanisms exist that can damage the renal allograft including the generation of free radicals, mechanical injury to blood vessels from sudden high blood flow, vasomotor derangement from prostaglandins and other regulatory peptide imbalances and cytokine release from inflammatory infiltrates (65).

\section{Risk factors related to the recipient}

Recipient age is a risk factor for DGF especially when kidneys from pediatric donors to adult recipients are involved (6). The relation between the occurrence of DGF and the discrepancy between donor and recipient Body Mass Indexes (BMI) supports this hypothesis (66).

The lower occurrence rate of DGF with zero HLA mismatch and low levels of panel reactive antibodies (PRA) (67), suggests that immune factors are responsible for this relation. Because the studies that describe this effect, used dialysis treatment in the first week as their definition of DGF, it is conceivable that early acute rejection activity constitutes the missing link $(4,6,60)$. In addition, the use of calcineurin inhibitors is a riskfactor because their vasoconstrictive properties influence renal perfusion and enhance ischemic damage (68). 
Finally abnormalities in the calcium and phosphate metabolism are not uncommon in patients on the waiting list for a renal transplant. For instance secondary hyperparathyroidism has been associated with a higher incidence of DGF $(69,70)$. But studies on the effect of hypercalcemia and hyperparathyroidism in renal transplants are lacking.

\section{DGF and long-term graft survival}

The effect of DGF on short and long term patient and graft survival is unclear. Some authors reported an effect of DGF on graft survival $(6,71)$ while others did not or only found this effect when it coincided with the occurrence of acute rejection episodes (72). Recent data on the outcome of grafts from non heart beating donors have shown, that if strict selection criteria were applied with respect to donor age, warm ischemia time and duration of oliguria of the donor, long term graft survival was good, despite the high incidence of DGF (2). Brook et al (1).found that when long term graft survival of grafts from heart beating donors (HBDs) experiencing DGF are compared with grafts from non heart beating donors (NHBDs) with DGF, graft survival after 6 years of follow up is even better in the NHBD group

When the survival curves are closely analyzed it is striking that 1 year after transplantation the survival curves run parallel. This suggests that DGF has its effect in the first year posttransplantation, but has no negative impact on graft survival beyond the first year. This finding is supported by studies that analyzed risk factors on graft survival after 1 year and found that progression of chronic graft failure was mainly associated with donor age, creatinine clearance, proteinuria and the presence of hypertension in the recipient and not with the occurrence of DGF per se (73).

\section{DGF and renal function}

One of the enigmatic problems of DGF is why some grafts react with DGF and others do not whereas the risk profiles are comparable Furthermore the effect of DGF on long term graft survival is unclear.

\section{Slope and intercept}

We proposed a theory on the course of graft function after transplantation. We presumed two variables to be important in the final evolution of graft function over time. The intercept determines the boundaries within which graft function develops. We suppose that this intercept reflects the quality of the transplanted organ. The slope exemplifies the long term course of graft function. Using this concept we analyzed renal function of 654 patients that were transplanted between 1983 and 1997. The creatinine clearance at 6 months was used at the determinant of the intercept of the graph that describes graft function over time and was categorized as more or less than $50 \mathrm{ml} / \mathrm{min}$. Chronic decline in function, modeled by one or two least square fitted regression lines after 6 months, was determined as a negative slope significantly different from zero. We created four patterns of evolution of graft function over time (Fig 4). $41 \%$ of grafts resumed and maintained optimal function post im- 


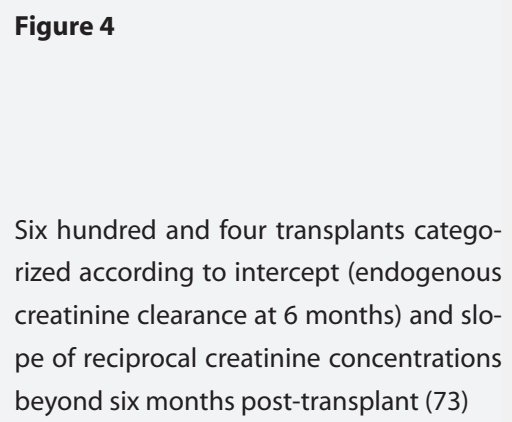

Figure 4

plantation (creatinine clearance $>50 \mathrm{ml} / \mathrm{min}$ ) whereas $28 \%$ achieved a similar function but experienced function deterioration afterwards (negative slope) The remaining $31 \%$ had a creatinine clearance at 6 months of $<50 \mathrm{ml} / \mathrm{min}$, of which half maintained stable function and the remaining grafts displayed progressive loss of function. Using logistic regression analysis we found that old donors and female gender of the donor, histoincompatibility ,the incidence of delayed graft function and the incidence of acute rejection episodes in the first 6 months were independent risk factors for a low intercept, whereas younger recipient age, previous sensitization, class I histoincompatibility, baseline immunosuppression and late acute rejection episodes were associated with a negative slope. In the multivariate analysis proteinuria and diastolic blood pressure at 6 months were determinants of graft function deterioration. We also found that the rate of deterioration is dependent on graft function at 6 or 12 months (73), in which grafts with a function of $<50 \mathrm{ml} / \mathrm{min}$ that deteriorate have a faster decline in renal function than grafts with a creatinine clearance of over $50 \mathrm{ml} / \mathrm{min}$.

\section{DGF and short term and long term graft function}

In a recent study (3), we analyzed the risk factors and the impact of DGF on graft loss and renal function. DGF was diagnosed, when serum creatinine level increased, remained unchanged or decreased less than $10 \%$ per day immediately after surgery during three consecutive days for more than one week excluding acute rejection when anti rejection treatment was started within this first week. Grafts that never functioned, ultimately leading to graft nephrectomy, were also excluded, because this is most often caused by surgical complications like renal vein or artery thrombosis or by hyperacute rejection. The incidence of DGF was related to a donor age of more than 50 years (odds ratio [OR] 2.21; confidence interval [Cl]:1.49-3.26), cold ischemia time (CIT) of more than 28 hours (OR 1.78; $\mathrm{Cl} 1.19-2.63)$, mean arterial pressure (MAP) of the donor of less than $100 \mathrm{mmHg}$ (OR 2.08; $\mathrm{Cl}: 1.43-3.03$ ) and the transplantation of a female donor kidney to a male recipient (OR 1.55; $\mathrm{Cl}: 1.15-2.55)$

Analyzing the impact of DGF on graft survival and graft function we found that DGF was associated with a suboptimal 1 year graft function but neither with inferior long term nor short term graft survival. Suboptimal graft function after 1 year, defined as a serum creatinine clearance of less than $50 \mathrm{ml} / \mathrm{min}$ was apart from the incidence of DGF associated with risk factors that can be classified as non-immunological [donors over 50 years of age 
(OR 2.39; 95\% Cl: 1.61-3.57) or female donors (OR 1.99; 95\% Cl: 1.42-2.78)] and immunological (more than 1 acute rejection episodes in the first year [OR 2.66; 95\% Cl: 1.87-3.78], peak panel reactive antibody level of more than $50 \%$ [OR $1.67 ; 95 \% \mathrm{Cl}: 1.15-2.47$ ] and the number of shared Cross reactive groups major histocompatibility complex class I antigens (CREG ) [OR 1.65; 95\% Cl: 1.09-2.49]. Long term graft survival was related to graft function at one year and the number of acute rejection episodes or treatments during this first year (Fig.5).

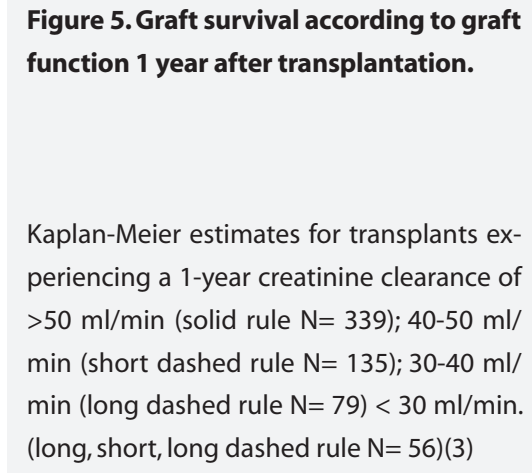

Figure 5. Graft survival according to graft function 1 year after transplantation.

Tubular Function Slope (TFS) in MAG-3 renal scintigraphy

To analyze the underlying functional mechanisms of DGF, we studied a group of 28 patients with immediate graft function (IGF) and 14 patients with DGF, with ${ }^{99 m}$ Technetiummercapto-acetyltriglycine (MAG-3) renography. The renal handling of MAG-3 is equivalent to paraminohippurate (PAH) (74). We defined TFS in a background subtracted MAG-3 graft curve: the first 2 minutes consist of a rapidly ascending phase as a result from initial perfusion and a second phase representing the tubular extraction phase. The slope of this second phase was defined as the tubular function slope TFS (Fig.6 ). Because this can only be done by active proximal tubular cells we used this TFS as a marker for functional renal mass. We found that all grafts had an initial recovery phase of the TFS from the moment of transplantation until a maximum level was reached after 3 to 4 weeks, indicating that initial damage from the transplantation procedure was comparable in both groups and that both groups recovered equally. However, in absolute terms, grafts with Immediate Graft Function (IGF) always had a higher TFS as compared with grafts with DGF and this difference persisted until the end of follow up at 3 years after transplantation. When creatinine clearance was analyzed for these grafts there also was a clear difference between the groups, but this did not reach the level of statistical significance (14). These findings suggested that grafts with DGF have an initial lower functional renal mass than grafts with IGF. Unfortunately this could not be translated in a difference in renal function, because of a lack of power of this study.or lack of accuracy in renal function estimation. 


\section{Figure.6}

a: Values of the tubular function slope (TFS) in the groups with and without DGF (14). After correction for the initial TFS value, the curves were superimposable, $(p=0.85)$ indicating that the differences between the two groups were determined by the differences already present in the early post transplant ${ }^{99 m}$ Tc-MAG3 renographies ( dashed rule, IGF ;solid rule, DGF).

b: Creatinine clearances at $3,6,12$ and 36 months after transplantation. The values tended to be lower in patients that experienced DGF but did not reach statistical difference ( dashed rule, IGF ;solid rule, DGF).

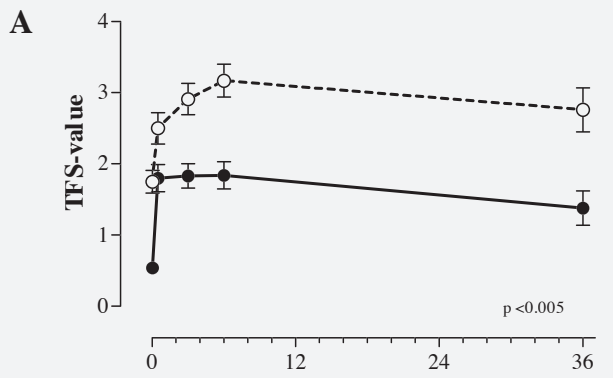

B

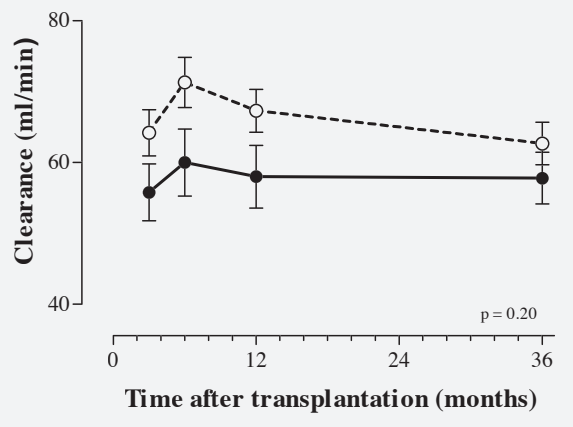

Figure.7 Creatinin clearances of grafts with and without DGF during five years follow-up.

a: At 3,6,12,24 and 60 months grafts experiencing DGF had lower creatinin clearances than grafts that did not.

b: After correction for the initial TFS value, the curves were superimposable.

( dashed rule ,IGF ;solid rule, DGF).
A

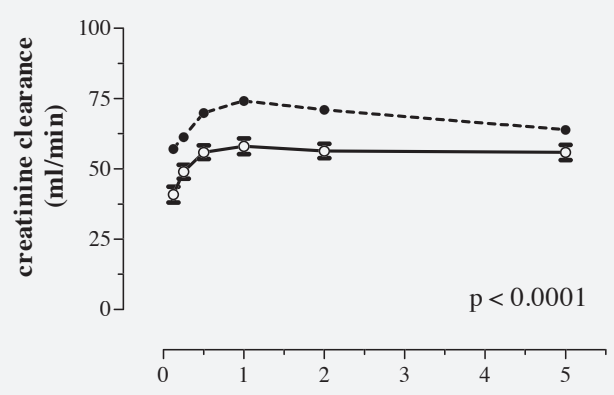

B

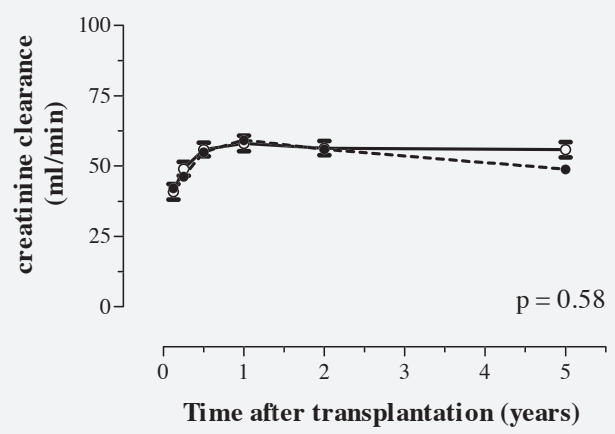


Therefore we expanded the groups with a population of seven hundred and ninety recipients of cadaveric renal transplants in the era 1983 until 1997 from a former study and analyzed creatinine clearances in a the IGF group and DGF group during a 5 years follow up period. This analysis confirmed that grafts with DGF had lower creatinine clearances than grafts that experience IGF, which was present during the whole period of follow-up $(p<0.0001)$. After correction for the initial difference in creatinine clearance between the 2 groups, the curves show a identical pattern over time $(p=0.58)$. There was a decrease in creatinine clearance at 5 years follow up, which was mainly present in the IGF group. The relatively better results in the DGF group at 5 years are presumably caused by the lower number of grafts in the DGF group after 5 years, and the loss of badly functioning grafts at that moment (Fig.7)

\section{Vulnerability and DGF}

\section{Damage versus protection}

The MAG-3 study gave us insight in the possible role of DGF in long-term graft function. We found that all grafts irrespective whether they develop DGF or not, experience an episode shortly after transplantation of impaired tubular extraction of MAG-3 that recovers within 3 weeks. This initial deprivation is caused by ischemia and subsequent tubular dysfunction, apoptosis and necrosis characteristic for the initiation phase of acute tubular necrosis (15) and are identical for grafts with and without DGF. However, the reason why a graft responds to this initial peri-transplantation injury with anuria, remains to be explained:Kidneys have a capacity to resist or to recover from ischemic or chemical induced tubular necrosis. These protective and repair mechanisms are characteristic for the maintenance and recovery phase of acute renal failure in native kidneys. Well known enzymes that are involved in tissue protection against ischemia and reperfusion injury are the (SODs)(75) and the hemoxygenase-1 (HO-1)(76,77). Theoretically grafts experiencing DGF have less protective and recovering capacity and therefore are more vulnerable to ischemic damage. A possible explanation for the difference between grafts reacting with DGF and grafts with immediate graft function lies in their ability to protect itself against ischemic and other damage during the whole transplantation procedure.

We therefore compared the expression of superoxide dismutases between grafts with and without DGF. We found that when manganese SOD was present, DGF occurs less frequently (Fig 8), whereas the presence of Cupper/Zinc and extra-cellular SOD had no effect. No relations were found between the incidence of the histological presence of ATN and the occurrence of DGF. However the presence of ATN correlated with a more extensive presence of active caspase- 3 staining. These findings support the idea that the way a kidney reacts on ischemia and reperfusion injury depends on the quality of the transplanted tissue, which is represented by its protective enzymes. However, clinical trials with the use of rh-SOD in humans have been disappointing with respect to the incidence of DGF $(32,78)$. Nevertheless a protective effective effect of treatment with Rh-SOD on long-term graft survival was found(78). Also a protective effect of hemo oxygenase-1 (HO-1) in rats has been found in vivo models with rats $(79,80)$. 
Figure 8
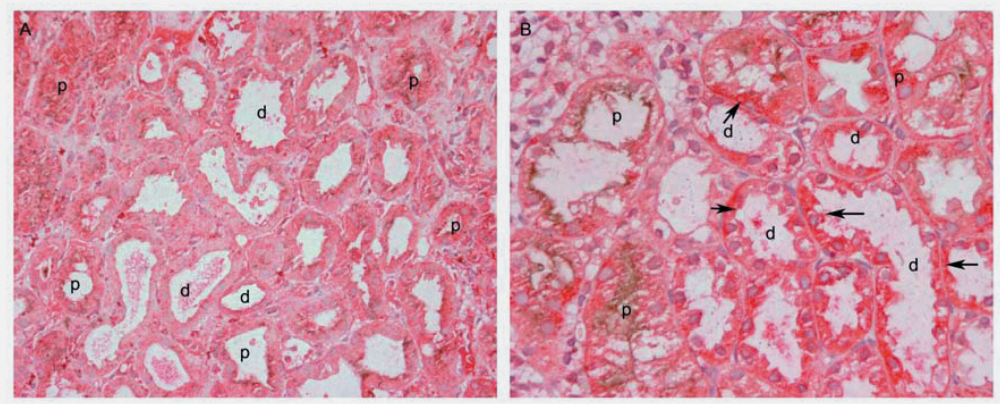

Distal tubular localization of Mn SOD in the presence (A) and absence (B) of DGF. Note the increased staining intensity of Mn SOD (red) in biopsies with DGF (right arrow). (Counter staining: anti-cubulin (brown), identifying the brush border of proximal tubules. p, proximal tubules; d, distal tubules.

Original magnification: 400x)

\section{Calcium and calcium channel blockers and DGF}

The role of calcium channel blockers in the protection against DGF is controversial (81-85). The protective role of CCB's against DGF is thought to be due to its vasodilatory capacity, which counteracts the vasoconstrictive effects of the calcineurin inhibitors, cyclosporine A or tacrolimus. Disturbance of calcium homeostasis is important in the pathogenesis of ATN.

Cytosolic calcium is a co-factor in the activation of cystein proteases like calpain and caspase-3 (86-88), enzymes that have an important role in the process of ATN. Acute renal failure in native kidneys has been reported in patients with serum calcium levels above 3.5 $\mathrm{mmol} / \mathrm{L}$. In native kidneys, acute renal failure due to hypercalcemia is describer in the milk alkali syndrome (89), severe hyperparathyroidism or PTH-related proteins (PTHrP) associated conditions(90) or multiple myeloma (91) and vitamin D intoxication (92). Little is known about the effects of hypercalcemia on the initial function of renal allografts. Torregosa et al. and Traindl et al. $(69,70)$ reported a significant effect of elevated PTH levels on the incidence of DGF, whereas serum vitamin D levels and serum calcium levels did not differ between the group with immediate graft function and the group with DGF.

The underlying mechanism explaining how calcium causes acute renal failure remains unresolved, but nephrocalcinosis may play a role. In animal models 3 types of nephrocalcinosis can be distinguished: chemical nephrocalcinosis, microscopic nephrocalcinosis and macroscopic nephrocalcinosis (93). The latter is characterized by gross calcium deposits found on radiographic investigations. Microscopic nephrocalcinosis is characterized by microscopic calcium deposits, mainly located in the lumen of the tubules. It is supposed to be a transitional phase between chemical and macroscopic nephrocalcinosis. Microscopic nephrocalcinosis is associated with increased calcium $x$ phosphate product and with chronic renal failure. Its effect on renal function is thought to be caused by tubular obstruction and tubular back-leak. Chemical nephrocalcinosis, assumed when macroscopic and microscopic are excluded, affects glomerular filtration rate by vasoconstriction and natriuresis 
induced volume constriction (94).It is histological characterized by areas of focal necrosis in the distal tubules and medullary collecting duct. High calcium content of the medullary area was found and the functional substrate was characterized by impaired function of the distal tubules. The role of cytoplasmatic calcium as an intracellular messenger in many important cell functions might explain these functional changes associated with the high cytoplasmatic calcium content. Calcium dependent enzymes that were analyzed in vitro in this respect are the cystein proteases, like calpaine and the caspases (20).

We therefore performed a study in which we analyzed the role of hypercalcemia in the occurrence of DGF. Serum calcium levels were correlated with the incidence of DGF in relation to other well known risk factors for DGF like donor age and cold ischemia time Furthermore the presence of calcium deposits in renal biopsies were correlated with the presence of DGF and serum calcium levels.

We found that hypercalcemia was an independent risk factor for DGF and that the pretransplant use of calcium channel blockers had a protective effect on the incidence of DGF. DGF was not associated with the presence of calcium deposits in renal biopsies, suggesting calcium influences tubular function by chemical nephrocalcinosis (95). This also suggested that the protective effect of calcium channel blockers is not only due to the formerly mentioned mechanisms but also by the prevention of the uncontrolled influx of calcium into tubular cells and subsequent activation of cystein proteases.

\section{DGF as a herald rather than a risk factor for poor long term outcome}

DGF in renal transplantation is a syndrome that is caused by ischemia and reperfusion injury. This syndrome of acute renal failure is a result of the interaction between the defense mechanisms of the graft on the one hand and the ischemic insults during the whole transplantation procedure on the other hand. Because these ischemic insults do not fully explain the graft's reaction upon transplantation, the differences in the defense mechanisms, which can be translated as differences in quality of the graft, probably are the key to the explanation why a graft reacts with DGF or not.

The often found relation between DGF and poor graft outcome therefore should also be explained in this scope of differences in quality of the graft, rather than that DGF is an independent risk factor for long-term graft outcome. The following findings support this hypothesis: First of all, we found that DGF was associated with a low intercept rather than with a negative slope in the curves that describe the evolution of graft function over time. Furthermore we showed during a follow up of 5 years that grafts from heart beating donors (HBD) reacting with DGF have an inferior long term graft function than grafts that do not react with DGF. Because this discrepancy was already present shortly after transplantation, we presume that differences in graft quality are also responsible for this phenomenon. The quality of the graft in essence determines, the full functional potentials of the graft which are determined by traditional risk factors for DGF, of which donor age and donor gender are important determinants. We found other arguments for our hypothesis in a MAG-III renography study in which a determinant of the functional renal mass, the tubular function slope (TFS), was found to be significantly lower during a follow up period of 3 years, in the DGF group compared to the non-DGF group. This difference was also already present 
shortly after transplantation. In an immuno-histochemical study we found a higher tissue expression of Mn SOD, a protective enzyme against ischemia and reperfusion injury and a possible marker of graft quality, in grafts experiencing immediate function.

Other risk factors like cold ischemia time, brain death of the donor, the use vaso-active agents in the procurement of the donor and warm ischemia time represent the amount of ischemic insults that the graft must resist. When these ischemic insults are large enough also a graft with a good quality ultimately will react with DGF. However when the graft overcomes this insult, its function should recover to a level that corresponds with its intrinsic quality. Findings in non-heart beating donations (NHBD) in which warm ischemia is prolonged as a representative of the intensified ischemic insults, illustrate that unless a high incidence of DGF, long term graft function and survival is equal in the NHBD and HBD group. Strikingly in the NHBD group donor age was lower and male gender more frequent. This may explain the comparable outcomes between the two groups, because this is determined by organ quality is guaranteed $(1,2)$. In heart beating donations we showed that the difference in function expressed as creatinine clearance between grafts experiencing DGF or not, is already present shortly after transplantation. Therefore it is not amazing that grafts experiencing DGF have a lower function during follow up. This means that DGF is an expression of poor quality rather than a cause of this graft deterioration.

\section{Conclusion}

DGF in renal transplantation is a syndrome that depends largely on the quality of the transplanted organ. Because the number of nephrons or the functional renal mass that is transplanted is determined at the time of the transplantation, the frame work within which graft function develops is set in the early post-transplant period. This functional renal mass is determined by factors like donor age and donor gender and this explains why these traditional risk factors are related with graft outcome. The occurrence of DGF is determined by the balance between ischemia reperfusion injury on the one hand and the functional renal mass and its protective mechanisms on the other hand.

Renal function is inversely correlated with the incidence of cardio vascular events and the main cause of patient death on hemo- and peritoneal dialysis is cardio vascular disease, this is of extreme importance in our search to solve the problem of organ donor shortage. Despite increased donor awareness and actions, the actual number of organ donors has not increased to any meaningful extend. This disparity has led to an increased interest in the use marginal donors, including donors at the extremes of age, donors with a history of hypertension or diabetes and non heart beating donations. To use these donors in an appropriate way in order to achieve a high percentage of transplantation in patients with end stage renal disease and above all good graft function, the quality of the graft should be assessed at the time of donation. Separate from a strict risk stratification in the case of non heart beating donation (96) and the use of histo-morphological criteria (97), measurement of MnSOD in the pretransplant biopsy might be of additive value to determine the quality of the graft. After having assessed this quality to some extend the ischemic insults to grafts with inferior function should be minimized. This can be done by minimizing the ischemic insults limiting cold and warm ischemia time and the use of a proper immunosuppressive 
regimen. The peri-operative use of calcium channel blockers within the framework of ischemia reperfusion injury should be reevaluated. On the other hand interventions should be developed to augment the intrinsic resistance against ischemic reperfusion injury. The application of protective enzymes like manganese Super Oxide Dismutase (Mn-SOD) and Heme-Oxygenase-1 (HO-1) should be considered. 


\section{REFERENCES}

1. Brook NR, White SA, Waller JR, Veitch PS, Nicholson ML. Non-heart beating donor kidneys with delayed graft function have superior graft survival compared with conventional heart-beating donor kidneys that develop delayed graft function. Am J Transplant 2003:3: 614-618.

2. Sanchez-Fructuoso Al, Prats $D$, Torrente J et al. Renal transplantation from non-heart beating donors: a promising alternative to enlarge the donor pool. J Am Soc Nephrol 2000:11:350-358.

3. Boom H, Mallat MJ, De Fijter JW, Zwinderman AH, Paul LC. Delayed graft function influences renal function, but not survival. Kidney Int 2000:58: 859-866.

4. Koning $\mathrm{OHJ}$, Bockel van JH, Woude vd FJ, Persijn GG, Hermans J, Ploeg RJ. Risk factors for delayed graft function in University of Wisconsin solution preserved kidneys from multiorgan donors. Transplant Proc 1995:27: 752-753.

5. Moreso F, Seron D, Gilvernet S et al. Donor age and delayed graft function as predictors of renal allograft survival in rejection-free patients. Nephrol Dialysis Transplant 1999:14:930-935.

6. Ojo AO, Wolfe RA, Held PhJ, Port FK, Schmouder RL. Delayed graft function: risk factors and Implications for renal allograft survival.Transplantation 1997:63: 968-974.

7. Halloran PF, Homik J, Goes $\mathrm{N}$ et al. The "injury response": a concept linking nonspecific injury, acute rejection, and long-term transplant outcomes. Transplant Proc 1997:29: 79-81.

8. Kahn D, Botha JF, Pascoe MD, Pontin AR, Halkett J, Tandon V. Withdrawal of cyclosporine in renal transplant recipients with acute tubular necrosis improves renal function. Transpl Int 2000:13 Suppl 1:S823.: S82-S83.

9. Giralclasse $M$, Hourmant $M$, Cantarovich $D$ et al. Delayed graft function of more than six days strongly decreases long-term survival of transplanted kidneys. Kidney Int 1998:54: 972-978.

10. Shoskes DA, Cecka JM. Deleterious effects of delayed graft function in cadaveric renal transplant recipients independent of acute rejection. Transplantation 1998:66: 1697-1701.

11. Troppmann C, Gillingham KJ, Benedetti E et al. Delayed graft function, acute rejection and outcome after cadaveral renal transplantation. Transplantation 1995:59: 962-968.

12. Lehtonen SRK, Isoniemi HM, Salmela KT, Taskinen El, Von willebrand EO, Ahonen JP. Long-term graft outcome is not necessarily affected by delayed onset of graft function and early acute rejection. Transplantation 1997:64: 103-107.

13. Gjertson DW. A multi-factor analysis of kidney graft outcomes at one and five years posttransplantation: 1996 UNOS Update. Clin Transpl 1996:343-60:343-360.

14. El Maghraby TA, Boom H, Camps JA et al. Delayed graft function is characterized by reduced functional mass measured by ${ }^{\left({ }^{99 m}\right)}$ Technetium-mercaptoacetyltriglycine renography. Transplantation 2002:74: 203-208.

15. Sutton TA, Fisher CJ, Molitoris BA. Microvascular endothelial injury and dysfunction during ischemic acute renal failure. Kidney Int 2002:62: 1539-1549.

16. Lieberthal W, Levine JS. Mechanisms of apoptosis and its potential role in renal tubular epithelial cell injury. Am J Physiol 1996:271:F477-F488.

17. Weinberg JM. The cell biology of ischemic renal injury. Kidney Int 1991:39:476-500.

18. Molitoris B.A. Ischemia-induced loss of epithelial polarity: potent role of the actin skeleton. Am J Physiol 1991:260:F769-F778.

19. Kribben A, Wieder ED, Wetzels JF et al. Evidence for role of cytosolic free calcium in hypoxia-induced proximal tubule injury. J Clin Invest 1994:93: 1922-1929. 
20. Edelstein CL, Ling H., Schrier RW. The Nature of renal cell injury. Kidney Int 1997:51:1341-1351.

21. Granger DN. Role of xanthine oxidase and granulocytes in ischemia-reperfusion injury. Am J Physiol 1988:255: H1269-H1275.

22. Cheeseman K.H., Slater T.F. An introduction to free radical biochemistry. Br Med Bull 1993:49:481-493.

23. Granger DN, Korthuis RJ. Physiologic mechanisms of postischemic tissue injury. [Review]. Annu Rev Physiol 1995:57:311-332.

24. Zimmerman BJ, Granger DN. Mechanisms of reperfusion injury. Am J Med Sci 1994:307: 284-292.

25. Baud L, Ardaillou R. Involvement of reactive oxygen species in kidney damage. Br Med Bull 1993:49: 621-629.

26. Olsen S., Solez K. Acute Renal Failure in man: pathogenesis in light of new morphological data. Clinical Nephrology 1987:27:271-277.

27. Racusen LC. The histopathology of acute renal failure. New Horiz 1995:3: 662-668.

28. Solez K, Lilliane Morel-Maroger, Jean-Daniel Sraer. The Morphology of "Acute Tubular Necrosis" in man: Analysis of 57 Renal Biopsies and a comparison with the glycerol model. Medicine 1979:58:362-376.

29. Molitoris BA, Leiser J, Wagner MC. Role of the actin cytoskeleton in ischemia-induced cell injury and repair. Pediatr Nephrol 1997:11:761-767.

30. Morita K, Seki T, Nonomura K, Koyanagi T, Yoshioka M, Saito H. Changes in renal blood flow in response to sympathomimetics in the rat transplanted and denervated kidney. Int J Urol 1999:6:24-32.

31. Singh I, Gulati S, Orak JK, Singh AK. Expression of antioxidant enzymes in rat kidney during ischemiareperfusion injury. Mol Cell Biochem 1993:125: 97-104.

32. Pollak R., Andrisevic JH, Maddux MS, Gruber SA, Paller MS. A randomized double-blind trial of the use of human recombinant superoxide dismutase in renal transplantation. Transplantation 1993:55:57-60.

33. Schneeberger $\mathrm{H}$, Illner WD, Abendroth $\mathrm{D}$ et al. First clinical experiences with superoxide dismutase in kidney transplantation--results of a double-blind randomized study. Transplant Proc 1989:21: 12451246.

34. Grone HJ, Weber K, Grone E, Helmchen U, Osborn M. Coexpression of keratin and vimentin in damaged and regenerating tubular epithelia of the kidney. Am J Pathol 1987:129: 1-8.

35. Wallin A, Zhang G, Jones TW, Jaken S, Stevens JL. Mechanism of the nephrogenic repair response. Studies on proliferation and vimentin expression after 35S-1,2-dichlorovinyl-L-cysteine nephrotoxicity in vivo and in cultured proximal tubule epithelial cells. Lab Invest 1992:66:474-484.

36. Witzgall R, Brown D, Schwarz C, Bonventre JV. Localization of proliferating cell nuclear antigen, vimentin, c-Fos, and clusterin in the postischemic kidney. Evidence for a heterogenous genetic response among nephron segments, and a large pool of mitotically active and dedifferentiated cells. J Clin Invest 1994:93:2175-2188.

37. Miller SB, Martin DR, Kissane J, Hammerman MR. Rat models for clinical use of insulin-like growth factor I in acute renal failure. Am J Physiol 1994:266: F949-F956.

38. Wang S., Hirschberg R. Role of growth factors in acute renal failure. Nephrol Dial Transplant 1997:12: 1560-1563.

39. Verstrepen WA, Nouwen EJ, Yue XS, De Broe ME. Altered growth factor expression during toxic proximal tubular necrosis and regeneration. Kidney Int 1993:43: 1267-1279.

40. Humes HD, Cieslinski DA, Coimbra TM, Messana JM, Galvao C. Epidermal growth factor enhances renal tubule cell regeneration and repair and accelerates the recovery of renal function in postischemic acute renal failure. J Clin Invest 1989:84:1757-1761.

41. Kawaida K, Matsumoto K, Shimazu H, Nakamura T. Hepatocyte growth factor prevents acute renal failure and accelerates renal regeneration in mice. Proc Natl Acad Sci U S A 1994:91:4357-4361. 
42. Franklin SC, Moulton M, Sicard GA, Hammerman MR, Miller SB. Insulin-like growth factor I preserves renal function postoperatively. Am J Physiol 1997:272: F257-F259.

43. Safirstein R, Price PM, Saggi SJ, Harris RC. Changes in gene expression after temporary renal ischemia. Kidney Int 1990:37: 1515-1521.

44. Kale S, Karihaloo A, Clark PR, Kashgarian M, Krause DS, Cantley LG. Bone marrow stem cells contribute to repair of the ischemically injured renal tubule.J Clin Invest 2003:112:42-49.

45. Safirstein R. Gene expression in nephrotoxic and ischemic acute renal failure.J Am Soc Nephrol 1994:4: 1387-1395.

46. Baylis C, Schmidt R. The aging glomerulus. Semin Nephrol 1996:16:265-276.

47. Anderson S, Brenner BM. Effects of aging on the renal glomerulus. Am J Med 1986:80:435-442.

48. Epstein M. Aging and the kidney. J Am Soc Nephrol 1996:7: 1106-1122.

49. Kappel B, Olsen S. Cortical interstitial tissue and sclerosed glomeruli in the normal human kidney, related to age and sex. A quantitative study. Virchows Arch A Pathol Anat Histol 1980:387: 271-277.

50. Nyengaard JR, Bendtsen TF. Glomerular number and size in relation to age, kidney weight, and body surface in normal man. Anat Rec 1992:232: 194-201.

51. Seron D, Carrera M, Grino JM et al. Relationship between donor renal interstitial surface and posttransplant function. Nephrol Dial Transplant 1993:8:539-543.

52. Curschellas E, Landmann J, Durig M et al. Morphologic findings in "zero-hour" biopsies of renal transplants. Clin Nephrol 1991:36:215-222.

53. Marshall R, Ahsan N, Dhillon S, Holman M, Yang HC. Adverse effect of donor vasopressor support on immediate and one-year kidney allograft function. Surgery 1996:120:663-665.

54. Darby JM, Stein K, Grenvik A, Stuart SA. Approach to management of the heartbeating 'brain dead' organ donor. JAMA 1989:261:2222-2228.

55. Es v.A., Hermans J., Bockel v.H.J., Persijn G.G., Hooff J.P., Graeff J.de. Effect of Warm Ischemia Time and HLA ( $A$ en $B$ ) matching on renal cadaveric graft survival and rejection episodes. Transplantation 1983:36: 255-258.

56. Daemen JW, Kootstra G, Wijnen RM, Yin M, Heineman E. Nonheart-beating donors: the Maastricht experience. Clin Transpl 1994::303-16.: 303-316.

57. Peters ThG., Shaver TR, Ames JE, Santiago-Delpin EA, Jones KW, Blanton JW. Cold ischemia and outcome in 17.937 cadaveric kidney transplants. Transpl Immunol 1995:59: 191-196.

58. Neumayer H.H., Eis M., Link J., Muhlberg J., Wagner K. Factors influencing Primary Kidney Graft Function. Transplant Proc 1986:XVIII: 1013-1117.

59. Schmidt R., Kupin W., Dumler F., Venkat K.K., Mozes M. Influence of the Pretransplant Hematocrit level on Early Graft Function in Primary Cadaveric Renal Transplantation. Transplantation 1993:55: 10341040.

60. Kahan B., Mickey R., Flechner S.M. et al. Multivariate Analysis of risk Factors Impacting on Immediate and Eventual Cadaver Allograft Survival in Cyclosporine-Treated Recipients. Transplantation 1987:43: 65-70.

61. Belli L.S., De Carlis L., Del Favero E. et al.The role of donor and recipient factors in initial renal graft nonfunction. Transplant Proc 1988:XX:861-864.

62. Koning $\mathrm{OHJ}$, Ploeg RJ, van Bockel JH et al. Risk factors for delayed graft function in cadaveric kidney transplantation - A prospective study of renal function and graft survival after preservation with University of Wisconsin solution in multi-organ donors. Transplantation 1997:63: 1620-1628.

63. Pfaff WW, Howard RJ, Patton PR, Adams VR, Rosen CB, Reed Al. Delayed graft function after renal transplantation. Transplantation 1998:65:219-223. 
64. Ploeg R.J., Bockel v.H.J., Langendijk P.T.H. et al. Effect of preservation solution on results of cadaveric kidney transplantation. LANCET 1992:340: 129-137.

65. Parmar MS, Kjellstrand CM, Solez K, Halloran PF. Glomerular endothelial cell detachment in paired cadaver kidney transplants: evidence that some cadaver donors have pre-existing endothelial injury. Clin Transplant 1994:8: 120-127.

66. Feldman $\mathrm{HI}$, Fazio I, Roth D et al. Recipient body size and cadaveric renal allograft survival. J Am Soc Nephrol 1996:7: 151-157.

67. Matas AJ, Gillingham KJ, Elick BA et al. Risk factors for prolonged hospitalization after kidney transplants. Clin Transplant 1997:11:259-264.

68. Neumayer $\mathrm{HH}$, Kunzendorf $\mathrm{U}$, Schreiber M. Protective effects of calcium antagonists in human renal transplantation. Kidney Int Suppl 1992:36:S87-93.: S87-S93.

69. Traindl O, Langle F, Reading $S$ et al. Secondary hyperparathyroidism and acute tubular necrosis following renal transplantation. Nephrol Dial Transplant 1993:8: 173-176.

70. Torregosa, Campistol, Fenollasa, Montesinos, Romar, Martinez de Osaba. Secondary Hyperparathyroidism and Post-Transplant Acute Tubular Necrosis. Nephron 1996:73:67-72.

71. Shoskes DA, Halloran PF. Delayed graft function in renal transplantation: etiology, management and long-term significance. J Urol 1996:155: 1831-1840.

72. Troppmann C, Gillingham KJ, Gruessner RWG et al. Delayed graft function in the absence of rejection has no long-term impact. Transplantation 1996:61:1331-1337.

73. Sijpkens YW, Zwinderman AH, Mallat MJ, Boom H, De Fijter JW, Paul LC. Intercept and slope analysis of risk factors in chronic allograft nephropathy. Graft 2002:5: 108-113.

74. Bubeck B, Brandau W, Weber E, Kalble T, Parekh N, Georgi P. Pharmacokinetics of technetium-99m_MAG3 in humans. J Nucl Med 1990:31: 1285-1293.

75. Dobashi K, Ghosh B, Orak JK, Singh I, Singh AK. Kidney ischemia-reperfusion: modulation of antioxidant defenses. Mol Cell Biochem 2000:205: 1-11.

76. Kato H, Amersi F, Buelow R et al. Heme oxygenase-1 overexpression protects rat livers from ischemia/ reperfusion injury with extended cold preservation. Am J Transplant 2001:1:121-128.

77. Wagner M, Cadetg P, Ruf R, Mazzucchelli L, Ferrari P, Redaelli CA. Heme oxygenase-1 attenuates ischemia/reperfusion-induced apoptosis and improves survival in rat renal allografts. Kidney Int 2003:63: 1564-1573.

78. Land W, Schneeberger H., Schleibner S. et al. The beneficial effect of human recombinant superoxide dismutase on acute and chronic rejection events in recipients of cadaveric renal transplants. Transplantation 1994:2: 211-217.

79. Tullius SG, Nieminen-Kelha M, Buelow R et al. Inhibition of ischemia/reperfusion injury and chronic graft deterioration by a single-donor treatment with cobalt-protoporphyrin for the induction of heme oxygenase-1.Transplantation 2002:74: 591-598.

80. Blydt-Hansen TD, Katori M, Lassman C et al. Gene transfer-induced local heme oxygenase-1 overexpression protects rat kidney transplants from ischemia/reperfusion injury. J Am Soc Nephrol 2003:14: 745-754.

81. Frei U, Harms A, Bakovic-Alt R, Pichlmayr R, Koch KM. Calcium channel blockers for kidney protection.J Cardiovasc Pharmacol 1990:16 Suppl 6:S11-5.:S11-S15.

82. Chan L, Schrier RW. Effects of calcium channel blockers on renal function. Annu Rev Med 1990:41:289302:: 289-302.

83. Epstein M. Calcium antagonists and the kidney. Implications for renal protection. Am J Hypertens 1991:4:482S-486S. 
84. Lustig S, Shmueli D, Boner G et al. Gallopamil reduces the post-transplantation acute tubular necrosis in kidneys from aged donors. Isr J Med Sci 1996:32: 1249-1251.

85. van Riemsdijk IC, Mulder PG, De Fijter JW et al. Addition of isradipine (Lomir) results in a better renal function after kidney transplantation: a double-blind, randomized, placebo-controlled, multi-center study. Transplantation 2000:70: 122-126.

86. Edelstein CL, Wieder ED, Yaqoob MM et al. The role of cysteine proteases in hypoxia-induced rat renal proximal tubular injury. Proc Natl Acad Sci U S A 1995:92: 7662-7666.

87. Edelstein $\mathrm{CL}$, Yaqoob MM, Alkhunaizi $\mathrm{AM}$ et al. Modulation of hypoxia-induced calpain activity in rat renal proximal tubules. Kidney Int 1996:50: 1150-1157.

88. Edelstein CL, Shi Y, Schrier RW. Role of caspases in hypoxia-induced necrosis of rat renal proximal tubules. J Am Soc Nephrol 1999:10: 1940-1949.

89. Abreo K, Adlakha A, Kilpatrick S, Flanagan R, Webb R, Shakamuri S. The milk-alkali syndrome. A reversible form of acute renal failure. Arch Intern Med 1993:153: 1005-1010.

90. Abraham P, Ralston SH, Hewison M, Fraser WD, Bevan JS. Presentation of a PTHrP-secreting pancreatic neuroendocrine tumour, with hypercalcaemic crisis, pre-eclampsia, and renal failure. Postgrad Med J 2002:78: 752-753.

91. Rota S, Mougenot B, Baudouin B et al. Multiple myeloma and severe renal failure: a clinicopathologic study of outcome and prognosis in 34 patients. Medicine (Baltimore) 1987:66: 126-137.

92. Zawada ET, Jr., Sanderson EW, Rossing D, Ohrt D, Simmons J. Hypercalcemia and acute renal insufficiency in a 24-year-old white male with lung disease. Am J Nephrol 1986:6: 152-157.

93. Wrong O.Nephrocalcinosis. In:Davidsen AM, Cameron JS, Grunfeld JP, Kerr DNS, Ritz E, Winearls CG, eds. Oxford Textbook of Clinical Nephrology. Oxford, New York,Tokyo: Oxford University Press, 1998: 13751396.

94. Levi M, Ellis MA, Berl T. Control of renal hemodynamics and glomerular filtration rate in chronic hypercalcemia. Role of prostaglandins, renin-angiotensin system, and calcium. J Clin Invest 1983:71: 16241632.

95. Boom H, Mallat MJK, De Fijter JW, Paul LC, Bruijn JA, van Es LA. Calcium levels as a risk factor for delayed graft function. Transplantation 2004:77: 868-873.

96. Kootstra G, Daemen JH, Oomen AP. Categories of non-heart-beating donors. Transplant Proc 1995:27: 2893-2894.

97. Remuzzi G, Grinyo JM, Ruggenenti P et al. Early Experience with Dual Kidney Transplantation in Adults using Expanded Donor Criteria. Journal of the American Society of Nephrology 1999:10: 2591-2598.

98. Thadhani R., Pascual M., Bonventre J.V. Acute Renal Failure. New England Journal of Medicine 1996:334: 1448-1459. 
SUMMARY

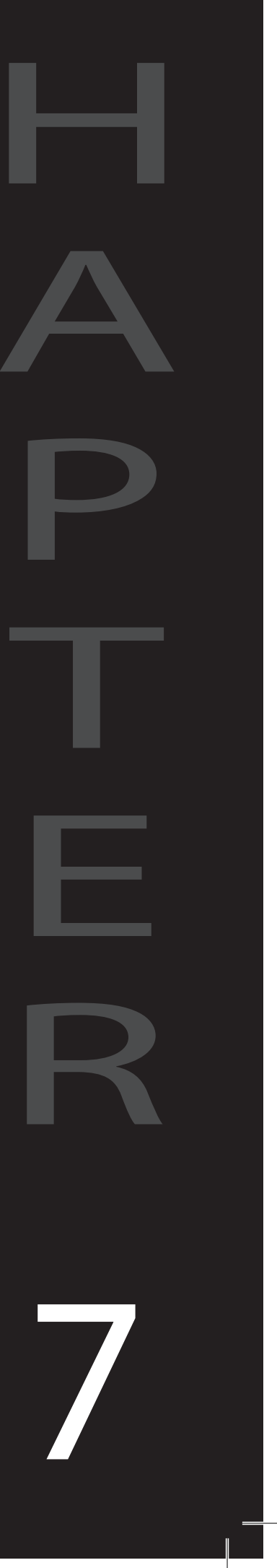


Delayed graft function (DGF) in renal transplantation remains enigmatic. Progress in the research after the etiology and consequences of DGF are hampered because DGF is a poorly defined syndrome and the clinical consequences of DGF on long term graft function have been difficult to ascertain. In general renal biopsies are not taken to document the cause of DGF but to exclude latent acute rejection episodes. Large biopsy studies on DGF are not consistently available. The interest in DGF has gained renewed interest with the increased use of marginal donors, including non-heart-beating donors, donors at the extremes of age, and donors with hypertension or diabetes. This group of donors experience DGF more frequently, with reported incidences of upto $50 \%$.

Our knowledge on DGF is mainly based on studies in experimental animals and on clinical data on acute renal failure in native kidneys (Chapter 1). This comparison has major flaws, since the risk factors and clinical setting for acute renal failure in the transplantation setting are substantially different from the risk factors for acute renal failure in native kidneys. In the transplant setting the kidney suffers from cold ischemia after the graft is harvested from the donor and cold perfusion is started. This procedure can last as long as 48 hours. Furthermore after the transplantation procedure ATN can be complicated by an increased likelihood of acute rejection episodes or drug-related nephrotoxicity.

\section{Pathogenesis of DGF}

Despite the above mentioned flaws, the underlying mechanism of DGF is considered to be related to ischemic and reperfusion damage, resulting in acute tubular necrosis.

In the pathogenesis of acute tubular necrosis, 3 stages can be recognized. The first stage is the ischemic phase in which ischemic and reperfusion injury (IRI) takes place and in which renal epithelial and endothelial cells are subjected to lethal insults leading to apoptosis and /or necrosis. The maintenance phase represents a phase of equilibrium between injury and intrinsic or up regulated defense mechanisms, events leading to cellular repair, proliferation and redifferentiation. This may lead to the recovery phase in which epithelial en endothelial function improve, leading to the recovery of renal function.

Lack of consistency in definition of DGF and its consequences for risk factors long term consequences

Risk factors for DGF are well known. These comprise older donor age, prolonged cold ischemia time and female gender of the donor. However there is debate on the impact of DGF on late graft outcome. Some authors reported an effect of DGF on renal allograft survival, while others only found inferior graft survival in patients who also experienced acute rejection episodes.

One possible explanation for this apparent difference in outcome may be the definition of DGF that is used. In most studies DGF is defined as the need of dialysis treatment in the first week after renal transplantation. This is a criterion that is easy to register and to obtain from large databases. However, dialysis during the first week after transplantation is also performed for other reasons than DGF, such as hyperkaliemia and / or fluid overload. 
Another flaw in this definition is the inability to exclude acute rejection and calcineurin inhibitor toxicity as an additional cause of impaired graft function. To study risk factors for DGF and its clinical consequences, it is therefore important to use a definition of DGF, in which the contribution of ischemia and reperfusion injury is stressed.

In this thesis we analyze the risks and consequences of delayed graft function, using a functional definition in which acute rejection and calcineurin inhibitor toxicity was excluded and the ischemic origin of DGF was stressed:

In this thesis we use a functional definition of DGF: We diagnosed DGF retrospectively, when serum creatinine level increased, remained unchanged or decreased less than $10 \%$ per day immediately after surgery during three consecutive days for more than one week excluding acute rejection when anti rejection treatment was started within this first week. Grafts that never functioned, ultimately leading to graft nephrectomy, were also excluded.

The above mentioned introduction is a summary of chapter 1 of the thesis

In chapter $\mathbf{2}$ we analyzed the risk factors for the occurrence of DGF in a cohort of 734 patients transplanted between 1983 and 1997. We found that the presence of DGF was associated with classical risk factors such as older donor age, a prolonged cold ischemia time and a low mean arterial blood pressure of the recipient. The impact of DGF was restricted to the quality of renal function (creatinine clearance) within the first year after transplantation but was not associated with inferior long term graft outcome. Besides the occurrence of DGF, one year renal allograft function was associated with older donor age, female gender of the donor and the occurrence of acute rejection episodes. Moreover, graft function after this first year is mainly determined by the quality of allograft function at one year and not with a history of DGF or acute rejection.

Using these findings, we hypothesized that, since graft function is correlated with the number of functioning nephrons (functional renal mass), DGF and long term graft function are related with each other through this functional renal mass. As it is known from literature that renal mass is influenced by age and gender, this functional mass might be the connecting link that explains the relation between DGF and poor graft outcome.

In chapter $\mathbf{3}$ we tested this hypothesis in a study that correlated the renal functional mass with long-term graft outcome. The functional renal mass was determined using the tubular function slope (TFS), a parameter in ${ }^{99 m}$ technetium mercaptoacetyltriglycine ${ }^{99 m}$ MAG3) scintigraphy. In a group of 42 grafts, 14 experienced DGF and had a significant lower functional renal mass than 28 grafts without DGF. This difference persisted during the total follow up period of 3 years. When the creatinine clearances were analyzed in the group of 734 patients described in chapter 2, the creatinine clearances in the DGF group were significantly lower during follow up period as compared with patients with immediate function of their transplanted kidney.

Another striking finding was that, in the DGF group as well as in the PF group, the functional renal mass was severely reduced directly after transplantation. Recovery took place within 1 month after transplantation and was identical for both groups. From these findings we conclude that ischemia and reperfusion injury leads to injury that is identical for all grafts. Its effect on functional renal mass of the graft disappeared within 1 month. 
Hypercalcemia is frequently seen in patients on renal replacement treatment. This is caused by the use of calcium containing phosphate binders and vitamin D analogues, in order to prevent hyperparathyroidism and related bone disease. However studies on the effect of hypercalcemia on DGF are lacking in literature. Hypercalcemia can result in renal failure in native kidneys (nephrocalcinosis), but the exact mechanism of how hypercalcemia leads to renal failure is not well known.

In experimental models 3 types of nephrocalcinosis are recognized:

1. Macroscopic nephrocalcinosis is characterized by calcium deposits that are recognized by ultrasound.

2. Microscopic nephrocalcinosis is characterized by the presence of calcium containing deposits in tubules, recognized by light microscopy.

3. Chemical nephrocalcinosis, is assumed when no calcium deposits are found in the presence of hypercalcemia and acute renal failure. Chemical nephrocalcinosis might be associated with high cytosolic calcium levels, that activate enzymes associated with necrosis and apoptosis.

In chapter $\mathbf{4}$ we described, to our knowledge, for the first time the relation between hypercalcemia and the occurrence of DGF in a group of 585 recipients of a cadaveric graft. We also looked for an anatomical substrate in 71 renal biopsies that were taken within the first week after transplantation. The presence of calcium crystals and signs of tubular necrosis did not correlate with serum calcium levels, suggesting that chemical nephrocalcinosis could be a determinant of DGF.

Another striking finding was that the use of calcium channel blockers (CCBs) protected against the occurrence of DGF. In the past these CCBs were frequently studied with the idea that its vasodilatory characteristics protected against calcineurin inhibitor toxicity. The results of these studies were not uniform and therefore CCB's are not used in clinical practice to prevent calcineurine inhibitor toxicity on a regular base. We found in a group of patients in which by definition calcineurin toxicity was excluded as a cause of DGF that the use of CCBs protected against the occurrence of DGF. This is probably explained by the prevention of high cytosolic calcium levels and subsequent activation of enzymes involved in the process of necrosis and apoptosis, like calpaines and caspases, involved in the pathogenesis of ATN.

Whether DGF develops in the allograft, depends on the balance between apoptosis and necrosis inducing factors on the one hand and protective factors on the other hand. In our opinion these protective factors are important determinants the quality of the graft and consequently long term graft outcome. The superoxide dismutases (SOD) are enzymes that act as scavengers of radicals that are produced upon ischemia and reperfusion damage.

In chapter $\mathbf{5}$ markers that are representative of the three stages in the development of ATN are studied in renal biopsies from grafts with and without DGF. Damage factors that dominate the initiation phase were visualized by morphological examination and by staining for active caspase 3, which is a major effector enzyme in the process of apoptosis. The protective response, that is important in the maintenance phase, was visualized by manganese SOD (Mn SOD). Signs of regeneration and proliferation, characteristic for the regeneration 
phase are characterized by vimentin and Ki-67 staining respectively. We found that grafts without DGF had a more intensive staining for Mn SOD. The presence of tubular necrosis was, as expected, associated with active caspase- 3 staining. The presence of vimentin and Ki 67 was associated with recovery of renal function but these data do not reach the level of significance. These findings support the hypothesis that the occurrence of DGF was the resultant of the balance between protective and damaging factors. Since the occurrence of DGF is associated with older donor age and female gender of the donor, the connecting link between these epidemiological and immunohistochemical factors, probably is the quality in terms of functioning nephrons of the graft. We propose that the presence of Manganese SOD in a pre-transplant biopsy can help to determine graft quality. Supplementation of Manganese SOD to grafts might be a method to diminish the occurrence of DGF and preserve renal allograft function. 


\section{SAMENVATTING}

Het vertraagd op gang komen van een nier die net getransplanteerd is, of te wel delayed graft function (DGF), wordt in de literatuur met een frequentie tot $50 \%$ van de getransplanteerde nieren beschreven. De klinische implicatie van dit fenomeen is dat patiënten na de transplantatie vaak alsnog gedialyseerd moeten worden en dat er weefselmonsters (biopten) uit de getransplanteerde nier moeten worden genomen om bijvoorbeeld acute afstoting (rejectie) of de bijwerking van geneesmiddelen uit te sluiten. Hierdoor verblijven patiënten met een vertraagd op gang komende nier gemiddeld langer in het ziekenhuis dan patiënten van wie de nier wel direct na de transplantatie goed functioneert. De nieren met DGF komen uiteindelijk altijd op gang en uiteindelijk hoeft de getransplanteerde patiënt niet meer gedialyseerd te worden.

Als onderliggend mechanisme voor DGF wordt zgn. ischemie- en reperfusie (I/R)schade verondersteld. Deze treedt tijdens de transplantatieprocedure m.n. op als de bloedvoorziening van de transplantaatnier wordt hersteld, wanneer de donornier bij de ontvanger wordt geïmplanteerd. Donornieren worden over het gehele Eurotransplant gebied verspreid, waardoor het transport van een orgaan door Europa soms tot 40 uur kan oplopen. Hierdoor ontstaat een periode van bloed- en zuurstofloosheid, die ischemie- en reperfusieschade veroorzaakt. Als gevolg van de I/R schade worden de verzamelbuizen van de nier (tubuli) beschadigd. Dit wordt acute tubulus necrose (ATN) genoemd, waarna de functie van de nier uiteindelijk weg valt. De kennis over de ontwikkelingsfasen bij ATN is met name gebaseerd op gegevens uit dierexperimenteel onderzoek en op een vergelijkbaar ziektebeeld bij niet-getransplanteerde patiënten, die op een Intensive Care afdeling worden opgenomen en van wie de nieren niet meer werken ten gevolge van ATN.

In deze modellen worden 3 fasen onderscheiden bij de ontwikkeling van acute tubulus necrose. Deze fasen kunnen gelijktijdig naast elkaar voorkomen. De eerste fase is de ischemische fase, een fase waarin als gevolg van het tekort aan zuurstof celdood optreed. Bij het optreden van deze celdood zijn enzymen betrokken zoals de calpainen en caspases die voor hun activiteit afhankelijk zijn van de aanwezigheid van calcium. De tweede fase is de fase waarbij een evenwicht bestaat tussen de bovengenoemde beschadigende factoren en de in de nier aanwezige beschermende factoren. Als uiteindelijk het herstel de overhand krijgt boven de beschadigende factoren, blijkt de nier een opmerkelijke mogelijkheid te hebben tot regeneratie. Deze derde fase van herstel wordt op weefselniveau gekenmerkt door snel delende cellen (mitoses) die kapotte dode cellen vervangen. Deze sneldelende cellen zijn waarschijnlijk stamcellen uit het nierweefsel of wellicht zelfs uit het beenmerg. Ze vertonen in deze korte tijd kenmerken van de gehele embryonale ontwikkeling van de nier, alvorens als volgroeide niercel te kunnen functioneren.

Over DGF bij de mens is in de transplantatie literatuur slechts weinig bekend. De bovengenoemde dierexperimentele kennis is niet zonder meer naar de humane situatie te vertalen, omdat er bij de transplantatiegeneeskunde veel oorzaken zijn waardoor een nier na de transplantatie niet op gang komt. Zo kan een acute rejectie (afstoting) of bijwerkingen van de immunosuppressiva (calcineurine remmer toxiciteit) een bijkomende oorzaak zijn voor het vertraagd op gang komen van de nier. Het is van belang om onderscheid te maken tussen de eerder genoemde I/R schade en deze twee laatste omstandigheden (acute afstoting 
en bijwerkingen van immunosuppresiva), omdat aan de laatste specifieke therapeutische consequenties verbonden zijn: Zo moet een acute afstoting behandeld worden en moet in het geval van calcineurine remmer toxiciteit de dosering van het geneesmiddel worden aangepast. Daarom worden er in de vroege periode na transplantatie met enige regelmaat biopten uit de net getransplanteerde nier genomen.

De betekenis van het optreden van DGF voor het functioneren van de getransplanteerde nier op de middellange en lange termijn is niet duidelijk. Zo zijn er onderzoekers die concluderen dat DGF geen effect heeft op duur dat de getransplanteerde nier functioneert (transplantaatoverleving). Anderen concluderen daarentegen dat DGF alleen van invloed op de transplantaatoverleving is als het in het beloop gevolgd wordt door het optreden van een acute afstoting reactie (rejectie). Weer anderen vinden dat als een nier na de transplantatie meer dan 1 week niet functioneert, de nier aanmerkelijk minder lang werkt dan als hij direct of binnen een week op gang komt (primaire functie (PF).

De reden dat er zo veel uiteenlopende meningen over de betekenis van DGF voor de lange termijn transplantaat overleving bestaan, is tweeërlei. In de eerste plaats bestaat er in de literatuur geen duidelijke en eenduidige definitie voor het syndroom DGF. Veel onderzoekers houden als criterium aan, of een patiënt na de transplantatie al dan niet gedialyseerd moet worden. Het nadeel van deze definitie is dat een getransplanteerde patiënt om een heel aantal andere redenen dan het niet op gang komen van de nier, gedialyseerd kan moeten worden. Zo komt het nogal eens voor dat een patiënt na de transplantatie dialyse behandeling behoeft vanwege een teveel aan vocht dat is toegediend tijdens de operatie of een te hoog kalium of fosfaat gehalte van het bloed.Zoals eerder gezegd kan het ook zo zijn dat een nier niet direct op gang komt vanwege het optreden van een acute afstoting of een te hoge dosering van de immunosuppressieve medicatie. Omdat dit allemaal aandoeningen zijn met verschillende oorzaken en toch allemaal worden aangeduid als DGF, is het niet verbazingwekkend dat de uitkomsten van verschillende onderzoeken niet eenduidig zijn.

In de tweede plaats was de interesse voor het klinische probleem van DGF nooit erg groot omdat het een aandoening is die in het algemeen vanzelf weer over gaat. Onderzoek naar de oorzaak van DGF werd om die reden dan ook zelden gedaan. Nierbiopten werden slechts genomen om acute afstoting of calcineurine remmer toxiciteit aan te tonen en niet om het onderliggende mechanisme voor het ontstaan van DGF te bestuderen. Hierdoor is er maar weinig menselijk weefsel voor onderzoek naar DGF beschikbaar.

De interesse voor DGF is de laatste jaren echter toegenomen. Dit komt omdat de wachtlijst voor een niertransplantatie aanzienlijk is verlengd, als gevolg van een dramatische afname van het aantal postmortale donoren de afgelopen 8 jaar. Daarom wordt er momenteel gezocht naar mogelijkheden om dit donortekort aan te vullen. Naast een toename van het aantal levende donaties, waarbij een familielid, vriend of kennis een nier aan een dialysepatiënt afstaat, wordt een deel van het probleem opgelost door de criteria voor het selecteren van een potentiële postmortale nierdonor aan te passen. Oudere donoren (dwz. donoren ouder dan 70 jaar) of donoren die een hoge bloeddruk of suikerziekte hebben, komen nu ook, onder bepaalde strikte voorwaarden, in aanmerking voor donatie. Daarnaast wordt er uitgebreid onderzocht of potentiële donoren die niet alleen hersendood maar ook een hartstilstand hebben gehad (non heart beating donation), in aanmerking komen voor donatie. Bij deze laatste twee groepen van donoren is het opvallend dat het aantal malen dat 
DGF optreedt aanzienlijk hoger is dan in de groep van bijvoorbeeld de bij leven gedoneerde nieren of de nieren die bij het constateren van de hersendood van de donor worden getransplanteerd (heart beating donation). We worden dus meer met DGF geconfronteerd en daarom is het opportuun om DGF en ATN in de humane situatie te onderzoeken.

Bovenstaande inleiding over delayed graft function bij niertransplantatie is een samenvatting van hoofdstuk 1 van dit proefschrift. Het doel van het vervolg van dit proefschrift is om met een duidelijke definitie van DGF, waarbij de I/R schade centraal staat en waarbij acute rejectie en calcineurine remmer toxiciteit zoveel mogelijk als oorzaak voor DGF zijn uitgesloten, de risicofactoren voor en de gevolgen van DGF voor het functioneren van de nier op de korte en lange termijn te definiëren. Op die mannier kunnen we uiteindelijk tot een hypothese komen over het mechanisme van DGF en hoe uiteindelijk de invloed van DGF is op de korte en lange termijn transplantaat functie.

In hoofdstuk 2 wordt een onderzoek beschreven waarbij in een cohort van 734 patiënten die tussen 1983 en 1997 werd getransplanteerd de risicofactoren voor het optreden van DGF werden vastgesteld. Het blijkt dat klassieke risicofactoren zoals een donorleeftijd boven de 50 jaar, een lage bloeddruk van de acceptor ten tijde van de transplantatie en een koude ischemie duur (KIT) van meer dan 28 uur, met name bepalen of een nier reageert met DGF. Een belangrijke bevinding is verder dat de duur dat een nier op de lange termijn functioneert, vooral afhankelijk is van de functie die na 1 jaar na de transplantatie is verkregen. Deze 1 jaars transplantaat functie blijkt m.n. afhankelijk te zijn van de leeftijd van de donor, het optreden van DGF en het aantal acute rejectie episodes die in dit eerste jaar zijn opgetreden. Dus de verdere ontwikkeling van de transplantaat functie na het eerste jaar blijkt verder niet afhankelijk te zijn van het optreden van DGF of het optreden een acute afstoting, maar alleen van de functie die na een jaar na transplantatie is bereikt.

Met deze gegevens is de hypothese opgesteld dat de nierfunctie bepaald wordt door de hoeveelheid functionerend nierweefsel (functionele renale massa). DGF en de nierfunctie op de lange termijn zij beide een uiting van nier functie en dus indirect ook een resultante van de functionele renale massa. Omdat uit de literatuur bekend is dat de renale massa wordt beïnvloed door factoren als leeftijd en geslacht zou het begrip 'functionele renale massa' de relatie tussen DGF en lange termijn transplantaat functie kunnen verklaren.

Om deze hypothese te toetsen wordt in hoofdstuk 3 een studie beschreven waarin de hoeveelheid functionerend nierweefsel wordt onderzocht in relatie tot het optreden van DGF en de lange termijn uitkomst. De hoeveelheid functionerend nierweefsel wordt middels een techniek uit de nucleaire geneeskunde ${ }^{99 m}$ technetium mercaptoacetyltriglycine ${ }^{99 m}$ MAG-3) scintigrafie) vast gelegd. In een groep van 42 getransplanteerde nieren blijken de 14 nieren die met DGF reageren, direct na de transplantatie een kleinere hoeveelheid functionerend nierweefsel te hebben dan de 28 nieren die direct op gang komen (primaire functie). Dit verschil blijft gedurende een follow-up periode van drie jaar bestaan.

Wanneer de creatinineklaring wordt berekend, als maat voor de transplantaatfunctie, blijft dit verschil ook gedurende deze follow-up periode van drie jaar, maar de significantie is net niet te bereiken. Daarom werd de analyse in de grotere groep die in hoofdstuk 1 is bestudeerd, opnieuw uitgevoerd. In deze groep bleek het verschil in creatinineklaring tussen de DGF en de PF groep, gedurende de gehele drie jaar follow-up, wél significant te zijn. Een ander opvallende bevinding is dat de functionele renale massa direct na de transplantatie in beide groepen (DGF én PF) verlaagd is en dat in beide groepen een identiek herstel 
patroon te zien is die na 3 tot 4 weken zijn volledige beslag heeft gekregen. Hieruit kan geconcludeerd worden dat de I/R schade voor alle getransplanteerde nieren hetzelfde is en er dus een zelfde periode van 3 a 4 weken nodig is om hier volledig van te herstellen. Een verhoogd serum calcium (hypercalciaemie) leidt bij niet getransplanteerde nieren tot nierfunctiestoornissen. Het precieze mechanisme hier achter is niet geheel duidelijk. Calcium is een ion dat in het lichaam in grote hoeveelheid aanwezig is De grootste hoeveelheid bevindt zich in het bloed, deels gebonden aan eiwitten en deels als vrij calcium en in de botten. De functie van calcium is gelegen in de bot op- en ombouw en daarnaast heeft het een belangrijke bijdrage in het op peil houden van de zuurgraad van het lichaam.. Verder is calcium werkzaam als een zogenaamde co-factor bij de activatie van tal van enzymen in het lichaam. Een groep van enzymen waar calcium een belangrijke co-factor voor is, zijn de enzymen die betrokken zijn bij het ontstaan van celdood (necrose) en geprogrammeerde celdood (apoptose), zoals calpaine en de caspases.

In dierexperimenteel onderzoek worden drie vormen van door calcium veroorzaakte nierfunctiestoornissen beschreven:

1. macroscopische nefrocalcinose, gekenmerkt door calciumzout neerslagen in het nierweefsel die middels echografie geïdentificeerd kunnen worden.

2. microscopische nefrocalcinose, gekenmerkt door de aanwezigheid van calciumzouten op microscopisch niveau.

3. chemische nefrocalcinose, wanneer in het geheel geen calciumzout neerslagen in het nierweefsel worden waargenomen. Deze laatste vorm van calcium gerelateerde nierfunctie stoornis wordt mogelijk veroorzaakt doordat calcium enzymatische processen onderhoudt, die uiteindelijk acute tubulus necrose veroorzaken.

Hypercalciaemie (te hoge calcium gehalte in het bloed) komt frequent voor bij patiënten die worden gedialyseerd. Dit heeft te maken met het frequente gebruik van calciumhoudende medicijnen (fosfaatbinders) en vitamine D. Deze medicijnen worden gebruikt om de nadelige gevolgen van te hard werkende bijschildklieren (hyperparathyreoidie) op botten en bloedvaten te verminderen. Deze hyperparathyreoidie treedt op als de nieren minder of niet meer werken. Echter, studies naar het verband tussen hypercalciaemie en het optreden van DGF na niertransplantatie zijn nooit gepubliceerd.

In hoofdstuk 4 beschrijven wij de voor het eerst in de literatuur gevonden relatie tussen het bestaan van hypercalciaemie en het ontstaan van DGF. Een andere opvallende bevinding is dat het gebruik van medicijnen die de calcium opname in cellen remt (calcium channel blockers (CCB's)), bescherming biedt tegen het optreden van DGF. Deze CCB's zijn in het verleden onderzocht met het idee dat zij door het verbeteren van de doorbloeding van de nier de nadelige werking van cyclosporine, een calcineurine remmer, konden tegengaan. De resultaten van deze studies zijn echter niet eenduidig. In de door ons onderzochte groep, waarin per definitie calcineurine remmer toxiciteit was uitgesloten, hebben deze CCB's wel een betekenis. Wellicht komt dit doordat CCB's voorkómen dat er hoge calciumconcentraties in de cel ontstaan waardoor chemische nefrocalcinose, en in aansluiting DGF, wordt voorkomen.

Daarnaast beschrijven wij in hoofdstuk 4 ons onderzoek naar een anatomisch substraat voor DGF en deze serum calciumwaarden in nierbiopten. Daartoe onderzochten wij 71 biopten die in de eerste week na transplantatie waren genomen en waarin geen acute rejectie of calcineurine remmer toxiciteit aanwezig was, op de aanwezigheid van calciumfosfaat 
kristallen. Deze bevindingen correleerden we met de serum calciumwaarden en tekenen van ATN. Wij vonden geen correlatie tussen de aanwezigheid van calciumneerslagen in het nierweefsel en de hoogte van de serum calciumwaarden. Dit ondersteunt de hypothese dat het calcium een andere rol heeft bij het ontstaan van DGF dan door de vorming van calcium zout neerslagen die meestal vermoed worden.

Om de balans tussen necrose en apoptose inducerende mechanismen aan de en kant en de aanwezigheid van beschermende factoren aan de andere kant te bestuderen, hebben wij in hoofdstuk 5 nierbiopten, die in de eerste week na transplantatie werden genomen, van patiënten met en zonder DGF, bestudeerd. De aanwezigheid van apoptose werd met behulp de markers TUNEL en caspase-3, gevisualiseerd. Daarnaast keken we of de aanwezigheid van beschermende factoren, zoals mangaan superoxide dismutase (Mn-SOD) geassocieerd is met het minder optreden van DGF of het vinden van ATN in deze biopten. Ten slotte keken wij of de aanwezigheid van vimentine en Ki 67, beide markers voor de regeneratie en het herstel van de nier, geassocieerd was met het optreden van DGF of de aanwezigheid van ATN. Wij vonden dat de expressie van actief caspase-3 verhoogd was als meer ATN in de biopten gevonden werd. Biopten uit transplantaten van patiënten die geen DGF hadden, vertoonden een hogere expressie van Mn-SOD dan die van patiënten met DGF. Deze bevindingen steunen onze hypothese dat een balans tussen beschadigende en beschermende factoren bepaalt of een getransplanteerde nier direct functioneert of niet. Het zou zo kunnen zijn dat de mate van aanwezigheid van Mn SOD een uiting is van de kwaliteit van het getransplanteerde orgaan en dat het niet alleen geassocieerd is met het minder optreden van DGF maar ook met een betere transplantaat overleving.

Hoofdstuk 6 is een review over DGF bij niertransplantatie, waarbij de huidige inzichten over pathofysiologie en klinische betekenis zijn weergegeven.

Samenvattend is onze hypothese dat delayed graft function (DGF) in de niertransplantatie een syndroom is dat voor het grootste deel bepaald wordt door de kwaliteit van het getransplanteerde orgaan. Omdat het aantal nephronen, of te wel de functionele renale massa, die initieel getransplanteerd wordt al bepaald is ten tijde van de transplantatie, zijn de grenzen waarbinnen de nierfunctie zich kan ontwikkelen al ten tijde van de ingreep en in de vroege periode hierna bepaald. De functionele renale massa is geassocieerd met determinanten zoals de leeftijd en geslacht van de donor. Daarom zijn deze risicofactoren niet alleen gerelateerd aan DGF maar ook aan het uiteindelijke lot van het transplantaat op de lange termijn.

Het optreden van DGF wordt bepaald door de balans tussen aan de ene kant ischemie- en reperfusieschade en de beschermende factoren aan de andere kant. De aanwezigheid van de beschermende factoren, die van nature aanwezig zijn of opgereguleerd worden zoals de superoxide dismutasen (SOD) kan worden samengevat als de kwaliteit van het orgaan en zou in de toekomst als leidraad kunnen fungeren bij de verdeling en toewijzing (allocatie) van organen. Zo kunnen de schaarse organen zo goed mogelijk worden gealloceerd, waardoor zo veel mogelijk mensen zo efficiënt mogelijk geholpen kunnen worden met een goed en langdurig werkend niertransplantaat. 


\section{ACKNOWLEDGEMENTS}

The studies described in this thesis have been performed at the department of Nephrology (Former head: Prof. L.C. Paul) of the Leiden University Medical Center.

The studies in this thesis were realized with the help of many people. Without their help this thesis could not have been written. Especially, I am grateful to all the patients who participated in the studies. Furthermore I want to thank Professor Paul, for introducing me into the world of transplantation science and clinical nephrology. Above all I want to express my appreciation for creating a very friendly atmosphere in the nephrology department, which is necessary for scientific but also the clinical work to prosper.

I thank professor van Es for spending so much time on this thesis with me. During the hours we spend together behind the microscope, I learned a lot, not only on renal morphology but also on things that are important in life.

I thank Annemieke van der Wal for the enormous amount of work she did like staining biopsies and by applying her knowledge and creativity to improve the results of the studies. Also Emile de Heer applied essential elements for the discussion and provided me with very valuable detailed information on various subjects of the study.

I thank the personnel of the laboratory of Nephrology (head: prof. Moh R. Daha) for teaching me patiently very basic laboratory techniques. Especially, I thank Maria Borrias Essers and Sandra v.d. Kooy for their expert technical assistance.

Of course I owe a lot of gratitude to the personnel of the outpatients' clinic, the nephrology clinical department and the secretariat of the Department of Nephrology. All contributed a lot to my joy of working in the LUMC.

I thank my father and my mother for giving me the opportunity to reach, my goals both socially and professionally.

My special gratitude goes to Janet, Oscar and Yizenne, for being so patient and encouraging me and above all still showing me their love. 


\section{CURRICULUM VITAE}

De auteur van dit proefschrift werd geboren op 21 maart 1963 te Koudekerk aan den Rijn. $\mathrm{Na}$ het behalen van het eindexamen VWO-B met klassieke talen in 1981, studeerde hij 2 jaar Fysiotherapie. In 1983 werd begonnen met de Studie Geneeskunde aan de Universiteit te Leiden. In 1988 werd het doctoraal diploma behaald en in 1990 het artsdiploma. In aansluiting werkte hij als vakantie assistent bij de afdeling Algemene Interne Geneeskunde (Prof. A.E. Meinders) van het LUMC. In 1991 werd gestart met de opleiding tot Algemeen Internist in het LUMC (Opleider: Prof. Dr. A.E. Meinders) en in 1997 werd hij als internist ingeschreven in het Specialisten Registratie Register.Van 1997 t/m 2001 werd hij opgeleid in het aandachtsgebied Nierziekten (Opleiders: Prof. Dr. L.A. van Es en Prof. Dr. L.C. Paul) in het Leids Universitair Medisch Centrum.

Gedurende deze periode werden ook de studies zoals die zijn weergegeven in dit proefschrift uitgevoerd. In 2001 werd hij geregistreerd als nefroloog. In 2002 was hij werkzaam als nefroloog in het Vlietland Ziekenhuis in Vlaardingen. Sinds 1 februari 2003 is hij als internist/nefroloog verbonden aan de Reinier de Graaf Groep in Delft. 